\title{
The Low Frequency Electrical Properties of Sea Ice
}

\author{
by
}

Sean Thomas Buchanan

\author{
A thesis \\ submitted to the Victoria University of Wellington \\ in fulfilment of the \\ requirements for the degree of \\ Master of Science \\ in Physics.
}

Victoria University of Wellington

2010 


\begin{abstract}
This thesis summarises an experimental and theoretical study of the low frequency electrical properties of sea ice. The aim of the research was to first demonstrate, and then gain a physical understanding of, the microstructural dependence of a sea ice impedance measurement. In particular, we sought to realise how the effective electrical properties of the medium depended on the volume fraction, orientation, dimensions, and connectivity of the dispersed brine phase.

The experimental portion of the project was performed on laboratory grown, artificial sea ice. We monitored the variation with time, and temperature, of the broadband sea ice impedance using four-electrode measurement cells embedded within the ice. The four-electrode measurement allowed us to realise and eliminate the contribution of electrode polarization to the measured impedance. By representing the electrical response of sea ice as a complex conductivity, we formulated a broadband physical model to describe the medium. The model distinguished bulk conduction, bulk polarization, and interfacial polarization. A complex non-linear least squares fitting procedure revealed the individual contribution of these physical processes and we studied their variation with temperature. We found that the bulk material underwent a dielectric relaxation with activation energy $E_{a}=0.20 \pm 0.04 \mathrm{eV}$. We linked the bulk material properties with a two phase microstructural model, with realistic input parameters.
\end{abstract}




\section{Acknowledgements}

This thesis would not have been possible without the support of a large number of people. Firstly, I thank my supervisors; Malcolm Ingham and Gideon Gouws for accompanying me on this scientific journey. Thank you for your good humour, for being approachable and easy to work with. I greatly valued your input. Thank you Malcolm for sending me to the other side of the world. Twice. I thank the other members, or intermittent, members of the VUW sea ice group: Keleigh Jones, Patrick Chote, Joe Trodahl and Rufus Boyack. Thank you Rufus for your continued interest in my research, your willingness to devote afternoons to EM problems and great friendship.

I thank the workshop guys at VUW: Alan Rennie, Manu PouajenBlakiston and more recently, Nick Grinter for constructing the dielectric array and numerous other bits and pieces (often at short notice). I thank Tim Exley for considerable assistance throughout the year with thermistors, cable fabrication, amplifier construction and for preparation of the Antarctic experiment. Thank you also Ann Hibbard for your work on testing the dielectric array.

I am grateful to Alex Gough and Andy Mahoney for installing the dielectric array and operating it throughout the Antarctic winter. Your prompt relay of data, scientific reports and technical issues was greatly appreciated. I thank Tim Haskell for facilitating Antarctic sea ice research and making the IRL cold room available for my use.

Thank you Darren Alexander, Kara Eaton, Dan Thompson and Liza Wilson for your administrative help.

I am grateful to Paul Chote and his macro lens for obtaining the excellent sea ice microstructure images shown on page 53.

I thank the 24 like minded souls with whom I spent three weeks in the high Arctic. Your company was more inspiring than the location. I thank the people that brought us together.

Thank you Mum and Dad for the thousands of hot meals and for 
putting up with this bloody obsession. Thank you to my friends for providing welcome distractions from this obsession.

This Masters year followed a four year physics degree at VUW. I thank all of those at VUW who, over this period, shared their knowledge, wisdom, skills and company with me. I am proud of the education I received here and would do it all over again, quite willingly. 
I am conscious of being only an individual struggling weakly against the stream of time. But it still remains in my power to contribute in such a way that, when the theory of gases is again revived, not too much will have to be rediscovered.

L. Boltzmann 


\section{Contents}

1 Introduction $\quad \mathbf{1}$

1.1 Sea ice ........................ 1

1.2 Previous Work . . . . . . . . . . . . . . . . . 4

1.3 Antarctic winter 2009 experiment . . . . . . . . . . . 5

1.4 Thesis Goal . . . . . . . . . . . . . . . . . . . . . . . 8

2 Dielectric theory and phase properties 9

2.1 Dielectric theory . . . . . . . . . . . . . . . 10

2.1.1 A Debye relaxation . . . . . . . . . . . . . 11

2.2 The electrical properties of brine . . . . . . . . . . . 12

2.3 The electrical properties of ice . . . . . . . . . . . . . . 15

2.4 Multiphase dielectric mixture theory . . . . . . . . . . . . 17

2.5 Space charge polarization . . . . . . . . . . . . . 19

3 Lab freezer experiment $\quad 21$

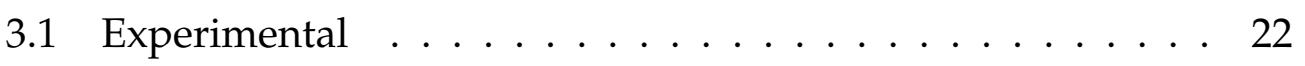

3.1 .1 Ice growth . . . . . . . . . . . . . . 22

3.1.2 Impedance measurement cells and instrumentation . 23

3.2 Results and analysis . . . . . . . . . . . . . 27

3.2.1 Evaluation of ice grown . . . . . . . . . . . 27

3.2.2 Impedance data . . . . . . . . . . . . . . . . . 29

3.2.3 Impedance measurement error . . . . . . . . . . . . 31

3.2.4 Permittivity data . . . . . . . . . . . . 32 
3.3 Discussion . . . . . . . . . . . . . . . . . . 35

3.3.1 Electrode polarization . . . . . . . . . . . . 35

3.3.2 High frequency impedance measurement error . . . 38

3.3.3 Gaining a physical insight . . . . . . . . . . . . . . 39

3.3.4 Realistic sea ice and non-invasive measurement cells 40

4 Wheelie bin experiment 41

4.1 Experimental description . . . . . . . . . . . . . . . . 41

4.1 .1 Growing realistic sea ice . . . . . . . . . . . . . . 4 41

4.1 .2 Temperature monitoring . . . . . . . . . . . . . 43

4.1.3 Impedance measurements using the four-electrode

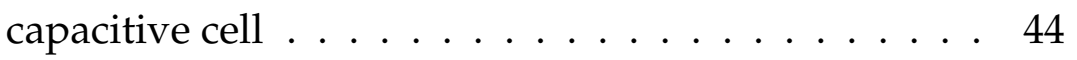

4.2 Results and Analysis . . . . . . . . . . . . . . . . 51

4.2.1 Temperature profile and observed sea ice microstructure ............... 51

4.2 .2 Sea ice impedance data . . . . . . . . . . . . . 54

4.2.3 Electrode polarization and the performance of the four-electrode capacitive cell . . . . . . . . . . 57

4.2.4 Sea ice permittivity data . . . . . . . . . . . . 60

4.3 Complex conductivity representation and a broadband physical model . . . . . . . . . . . . . . . . 63

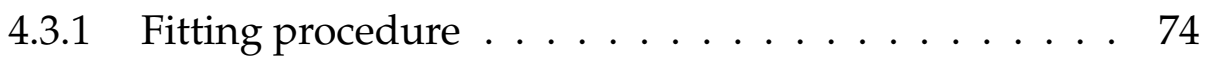

4.3.2 Bulk material processes . . . . . . . . . . 76

4.3.3 Interfacial polarization . . . . . . . . . . . . 83

4.4 Mixture model . . . . . . . . . . . . . . . . . . . . . 85

5 Summary and Perspective 93

5.1 The low frequency electrical properties of sea ice . . . . . . . 93

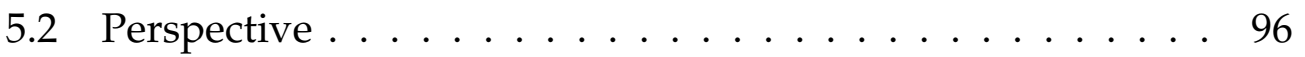

5.2.1 Electrode polarization . . . . . . . . . . . . . . 96

5.2 .2 Applicability . . . . . . . . . . . . . . 96

5.2.3 Impedance measurement error . . . . . . . . . . . 97 
6 Antarctic winter 2009 experiment 100

6.1 The field site and capacitor array deployment . . . . . . . . . 101

6.2 Impedance measurement data . . . . . . . . . . . . . . . 103

6.3 Interpretation . . . . . . . . . . . . . . 107

6.4 Future work . . . . . . . . . . . . . . . . . . 111

6.5 Conclusion . . . . . . . . . . . . . . . . . . . 112

$\begin{array}{ll}\text { A Impedance compensation } & 114\end{array}$

B Additional wheelie bin data 116

C A Debye relaxation in a lossy material 123

D Complex non-linear least squares fitting 127

D.1 Theory . . . . . . . . . . . . . . . . . . 127

D.2 6 parameter complex conductivity fit . . . . . . . . . . 128 


\section{Chapter 1}

\section{Introduction}

\subsection{Sea ice}

Sea ice is the frozen ocean which exists in the polar regions of the Earth. Its growth is forced, primarily, by heat loss from the ocean to the atmosphere, and its extent varies dramatically over the course of the year. During the Antarctic winter of 2009 the sea ice extent was largest, on average, for the month of August. It then covered an area of $19 \times 10^{6} \mathrm{~km}^{2}$ [National Snow and Ice Data Center (2010)] which is comparable in size to the Antarctic continent. Most of this sea ice melted over the warmer summer months. The minimum extent of $3 \times 10^{6} \mathrm{~km}^{2}$ occurred for the month of February. This remaining portion is known as multiyear sea ice. The portion that melts is first year sea ice.

Sea ice plays an important role in the global climate. Its high albedo means that a large fraction of the incident solar flux is reflected back into space. It insulates the underlying ocean from the atmosphere, and salt rejection from its base forms the deep water layer which completes global ocean circulation. This cold salty water sustains primary producing organisms, which become the food source of the worlds oceans.

Annual trends of reduction in seasonal sea ice extent have been observed in the Arctic [National Snow and Ice Data Center (2010)]. This 


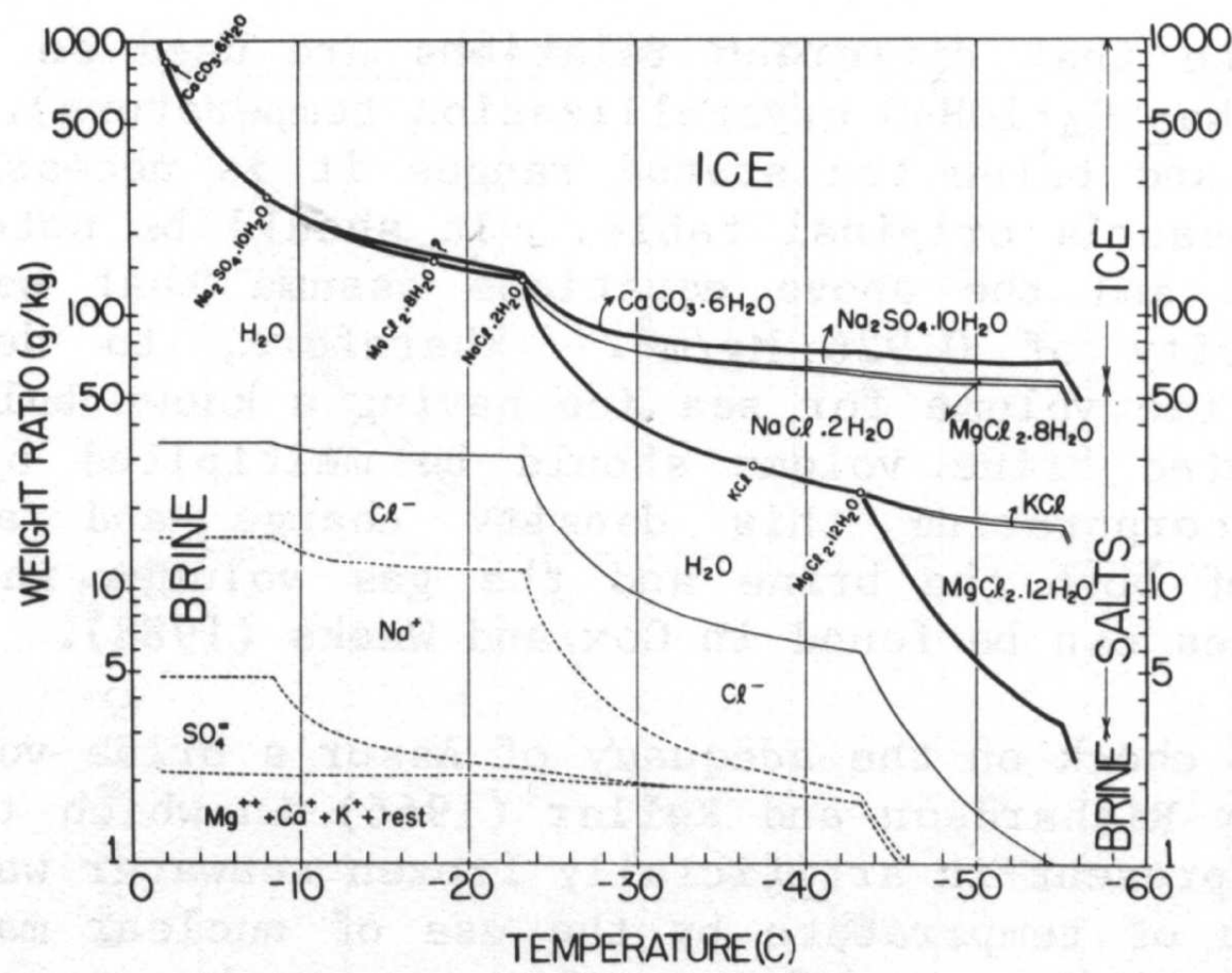

Figure 1.1: Phase diagram for sea ice. Reproduced from Assur (1958).

long term variation, coupled with its climatic and biological importance, necessitates scientific studies of sea ice.

Sea ice forms at the top of the ocean when sea water is cooled below its freezing point [Eicken (2003)]. This point is suppressed to approximately $-2^{\circ} \mathrm{C}$ by the presence of dissolved salts and the phase transition is not sudden. The ice crystals which form incorporate no, or very little, salt. The salinity of the remaining brine increases, further suppressing its freezing point. Figure 1.1 shows the equilibrium phase diagram of frozen sea water. The circles mark the temperature when salts precipitate out of solution. The brine volume fraction quantifies the ratio of the brine phase to the total volume.

In natural sea ice formation the majority of brine is dissipated into the 
underlying ocean. Some, however, becomes trapped in pockets or channels within the sea ice. The medium can be considered as a heterogeneous composite of ice, brine, air bubbles and solid salts. The microstructure of sea ice is the volume fraction, dimensions, orientation, and connectivity of the dispersed brine phase. This microstructure depends primarily on the sea ice temperature and how it was formed. Columnar ice describes sea ice with brine pockets which have a preferential vertical orientation. This ice is formed in calm waters by vertical heat loss.

Sea ice microstructure is important because it controls, or influences, the macroscopic properties of the ice [Eicken (2003)]. This influence includes the mediums energy transport properties: radiative, conductive and convective. For example, consider the thermal conductivity of sea ice. Brine channels facilitate thermal convection - a far more efficient heat transfer process than the conduction which occurs in a solid block of ice. If vertical brine channels span the sea ice then, the thermal conductivity is significantly increased. The mechanical properties, such as porosity and shear strength, are also controlled by the microstructure.

This macroscopic influence necessitates sea ice microstructural monitoring. The traditional method involves observing extracted cores under magnification. The core salinity and temperature are also measured. The problem with this method is that it is very involved, and ultimately destructive. As soon as the core is removed from the ice then it is exposed to a different temperature and pressure which could force melting or refreezing and/or brine drainage.

We consider electrical methods to be a technique capable of monitoring sea ice microstructure. This technique is appropriate because brine and ice have contrasting electrical properties: brine is a good electrical conductor, ice is not - the charge transfer mechanism in brine is ionic, in ice it is protonic. The advantage of electrical methods is that they can be performed on the ice in-situ and they offer the possibility of automated sea ice monitoring. Because the microstructure of sea ice can be anisotropic, 
the electrical properties can be anisotropic.

\subsection{Previous Work}

Previous studies of the electrical properties of sea ice fall into a number of categories. The first involves DC resistivity soundings performed with surface electrodes or electrodes embedded within the ice. The measurements are performed across large sections $(\sim 5 \mathrm{~m})$ of sea ice. The triumph of these studies is the two-dimensional resistivity tomography work by Ingham et al. (2008). This has demonstrated microstructural sensitivity by resolving anisotropy in Arctic sea ice [Jones et al. (2009)]. They observed a larger horizontal than vertical resistivity due to the columnar sea ice microstructure. This is because the vertical pockets provided an easy electrical conduction path. More recently this technique has identified a change from columnar to platelet sea ice, at depth, in Antarctic sea ice [Ingham and Jones, personal communication]. The technique has recently been extended to low frequency $\mathrm{AC}$ resistivity measurements which involve measuring a frequency dependent impedance with magnitude and phase. The technique has been applied in the laboratory [Gouws et al. (2010)] and the Antarctic [Ingham et al. (2010)].

Most studies on the electrical properties of sea ice have been performed at microwave frequencies $(\mathrm{GHz})$. This frequency range is applicable to remote sensing. The permittivity, which characterises the mediums electromagnetic response, depends on the microstructure of the sea ice. Knowledge of how this permittivity varies within the ice could allow sea ice reflections data to be interpreted in terms of sea ice thickness. The most comprehensive study of the permittivity of sea ice at high frequencies was performed by Vant et al. (1978).

Backstrom and Eicken (2006) and Pringle et al. (2009) performed $50 \mathrm{MHz}$ impedance measurements with commercial 'capacitance probes' on artificial and Arctic sea ice. They investigated the salinity monitoring capability 
of these sensors. Their work is open ended.

Some work has been undertaken on the low frequency electrical properties of small sea ice samples. The most comprehensive study was performed by Addison (1969). He conducted impedance measurements using parallel plate mesh capacitors on laboratory prepared saline ice of varying salinities. The measurements were performed across the frequency range $10 \mathrm{~Hz}-100 \mathrm{MHz}$ and at temperatures $-35^{\circ} \mathrm{C}$ to $-12.5^{\circ} \mathrm{C}$. The results were presented as complex permittivities. In a subsequent paper Addison (1970) studied the relaxation of this saline ice. He interpreted the broadband response in terms of a number of different physical processes.

Notz (2005) devised a field deployable salinity monitoring system. It functions by making $1 \mathrm{kHz}$ impedance measurements between $5 \mathrm{~mm}$ separated parallel platinum wires. The device has been deployed in Arctic sea ice to monitor the salinity in a refrozen borehole [Notz and Grae Worster (2008)].

\subsection{Antarctic winter 2009 experiment}

In 2009 a multi-institutional campaign to monitor winter sea ice growth in McMurdo Sound, Antarctica was undertaken. It included contributions from New Zealand's main sea ice research groups: the National Institute of Water and Atmospheric Sciences (NIWA), the University of Otago (UO) and Victoria University of Wellington (VUW). Alex Gough and Andy Mahoney of the University of Otago performed the field component of the campaign and spent the winter months maintaining a plethora of experiments within and below the McMurdo Sound sea ice.

The campaign presented a unique opportunity for sea ice impedance monitoring over a long period of the Antarctic winter growth, which could be followed up with measurements in spring by a team from VUW. Over the summer of 2008-2009 an experiment was prepared which could monitor the sea ice impedance at different depths within the ice and could 
be operated by the wintering-over crew. It consisted of a vertical array of six parallel plate capacitors which could be deployed through the sea ice surface. The sea ice would then form around this 'capacitor array'.

The capacitor array is depicted in Figure 1.2. The sensors were disc parallel plate capacitors made of marine grade stainless steel. The plate separation was approximately $25 \mathrm{~mm}$. The structure was held together by four vertical fibreglass rods fixed to a circular base. The vertical separation between the plates was irregular along the $2 \mathrm{~m}$ structure. The exact position of the capacitors is given in Figure 1.2. The capacitors are labelled from bottom to top, sensor 1 (S1) to sensor 6 (S6). S1, S3, S4 and S5 are orientated horizontally and S2 and S6 are orientated vertically. This aspect of the design was included to investigate impedance anisotropy. Capacitor S4 was different from the others in that it is made of mesh - this was an attempt to include a less intrusive type or measurement cell which, unlike solid plates, would facilitate brine drainage.

Impedance measurements between each of the capacitors could be made manually from the sea ice surface using a custom impedance analyser. The device is described in Gouws et al. (2009). It was capable of impedance measurements between $10 \mathrm{kHz}$ and $100 \mathrm{kHz}$. Silicon coated measurement cables connected the electrodes to the instrument via soldered ring connectors. The cables extended $3 \mathrm{~m}$ from the electrodes, running along opposing fibre glass rods, to a multi plug which connected to the instrument box. The instrument and the capacitor array underwent extensive laboratory testing.

This experiment was very much a prototype and it was sent to Antarctica with the knowledge that some aspects of the design may prove unsuitable for field measurements. Its performance would lead to a refined design. 


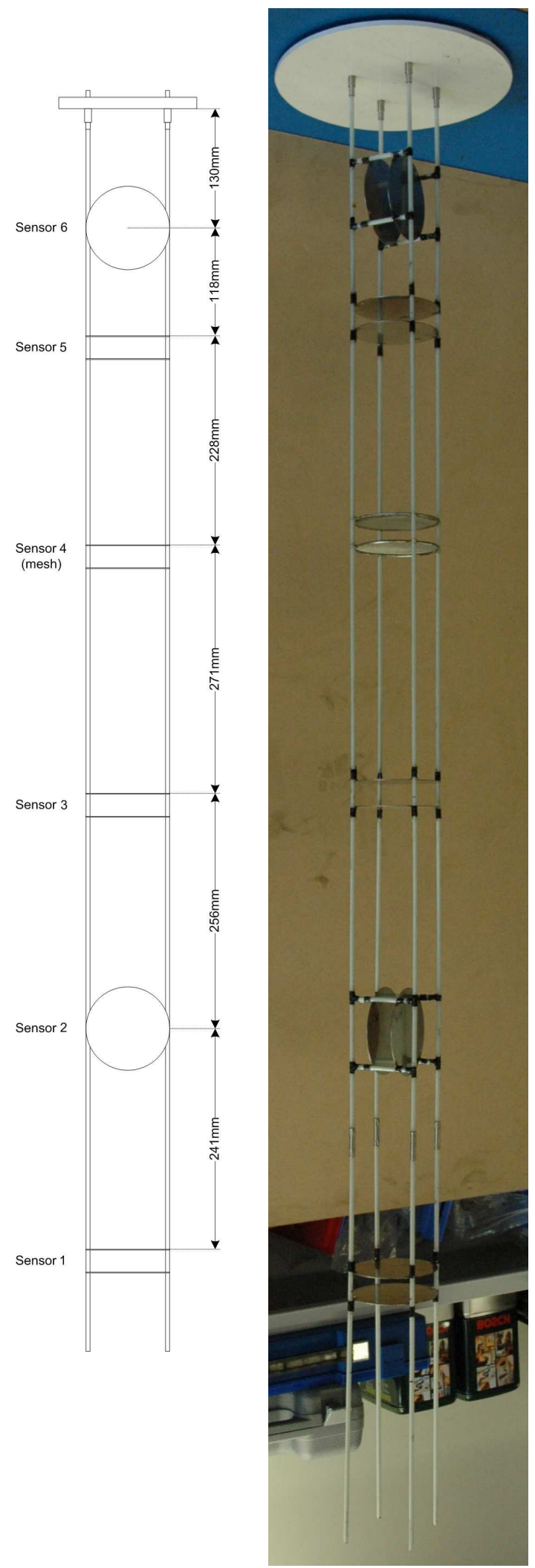

Figure 1.2: The capacitor array. 


\subsection{Thesis Goal}

By the time the Author began this MSc, the Antarctic experiment had been boxed and shipped to Antarctica. There were months to wait before the ocean froze and the impedance data began to trickle back. The goals of this MSc, however, were dictated entirely by this Antarctic experiment.

The goal of this MSc was to perform tangible laboratory measurements on synthetic sea ice. We attempted to recreate the Antarctic experiment in an idealised environment and perform impedance measurements to a precision probably not possible in the field. The experiment would produce a mass of reference data to aid in the interpretation of the field data. We also sought to gain a physical understanding of the low frequency electrical properties of sea ice and how they depend on sea ice microstructure. 


\section{Chapter 2}

\section{Dielectric theory and phase properties}

The electrical properties of ice and brine are contrasting: brine is an excellent electrical conductor, whereas ice is not - the charge transfer mechanism in brine is ionic, in ice it is protonic. This is the reason why electrical methods offer microstructural sensitivity. The electrical response of an ice/brine composite, depends on the electrical properties of the two phases, and the phase geometry.

One of the goals of this thesis was to interpret electrical measurements in terms of brine microstructure. This requires a physical understanding of the composite properties. Figure 2.1 previews some of the physical processes which might contribute to the electrical properties of sea ice.

Dielectric theory is the framework in which this story is told and this chapter begins with its formalisation. This section includes: a description of dielectric relaxation, the frequency dependent electrical properties of ice and brine, and finally, we consider how these phases interact and outline appropriate composite medium theories. 


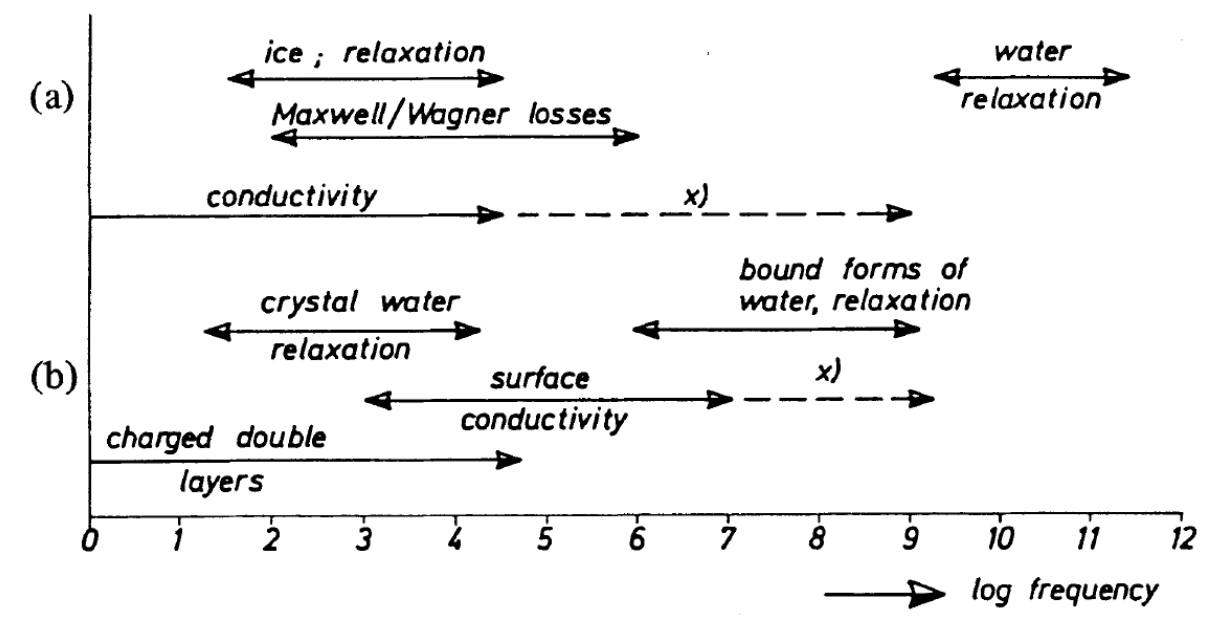

Figure 2.1: Survey of losses in heterogeneous mixtures containing water: (a) without interfaces, (b) with interfaces present. Reproduced from de Loor (1982).

\subsection{Dielectric theory}

A potential difference applied between two parallel electrodes produces an opposing charge distribution. If the plates are infinite in extent, then a homogeneous electric field orientated parallel to the plates results. The systems capacitance, $C_{0}$, characterises the capacity to store charge and it depends on the electrode geometry. It is given by:

$$
C_{0}=\frac{A}{d} \epsilon_{0}
$$

where $A$ is the plate area, $d$ is the plate separation, and $\epsilon_{0}$ is the permittivity of free space.

If a dielectric medium exists between the two plates then it becomes polarized. Additional charge can then be accommodated on the plates to compensate for this internal bound charge and the capacitance increases. It becomes $C=\epsilon C_{0}$, where $\epsilon$ is the dielectric constant or relative permittivity of the medium. It is typically frequency dependent.

A more general parameter which describes the response of a medium 
is the complex permittivity $\epsilon^{*}$.

$$
\epsilon^{*}=\epsilon^{\prime}-j \epsilon^{\prime \prime}
$$

where $j=\sqrt{-1}, \epsilon^{\prime}$ describes material polarization and $\epsilon^{\prime \prime}$ describes energy dissipation (loss).

In sea ice the material properties are anisotropic and $\epsilon^{*}$ is a rank two tensor.

\subsubsection{A Debye relaxation}

Debye (1945) produced a theory which accurately describes the frequency dependent electrical response of many materials. A Debye relaxation describes a polarization mechanism which becomes unviable at high frequencies. At intermediate frequencies a resonance occurs within the material producing large energy dissipation. The process is described by the Debye equation:

$$
\epsilon^{*}=\frac{\chi}{1+j \omega \tau}+\epsilon_{\infty}
$$

where $\chi$ is the susceptibility and represents the dielectric contribution of the low frequency polarization mechanism, $\omega$ is the angular frequency, $\tau$ is the relaxation time, and $\epsilon_{\infty}$ describes the residual polarization.

The theory is applicable to polar molecules. Given time, their dipole moment aligns with a polarizing field. This is represented by a large low frequency permittivity. At high frequencies the molecules do not have time to align and their dipoles do not contribute to the net polarization. The high frequency permittivity is due solely to electronic and atomic polarization. At intermediate frequencies, a resonance occurs. The molecules collide and dissipate energy - this is dielectric loss.

Both water and ice undergo Debye relaxations. They are also conductive mediums and have an electrical conductivity of $\sigma_{D C}$. The complex permittivity can represent both of these processes with the equation:

$$
\epsilon^{*}=\frac{\chi}{1+j \omega \tau}+\epsilon_{\infty}-j \frac{\sigma_{D C}}{\epsilon_{0} \omega} .
$$




\subsection{The electrical properties of brine}

The conductivity of sea water is due overwhelmingly to the migration of dissolved salts. Sea water of the worlds oceans has an average salinity of 35ppt. This means that every litre includes $35 \mathrm{~g}$ of what is primarily $\mathrm{Na}^{+}$and $\mathrm{Cl}^{-}$ions. The ions are free and respond readily to an applied electric field. Their differential movement constitutes an electrical current. Janz and Singer (1975) presented the temperature dependent electrical conductivity for standard sea water above $-2^{\circ} \mathrm{C}$. There results are shown in the high temperature portion of Figure 2.2.

At lower temperatures ice begins to form and the salinity of the remaining brine increases. In an equilibrium situation the salinity of sea ice brine depends only on the temperature. Stogryn and Desargant (1985), exhaustively, collected laboratory sea ice brine samples and made conductivity measurements at different brine temperatures $T$. Their work is summarised in the following equation and shown in the low temperature portion of Figure 2.2.

$$
\sigma_{D C}= \begin{cases}-T \exp (0.5193+0.08755 T) & \text { for } T \geq-22.9 \\ -T \exp (1.0334+0.1100 T) & \text { for } T<-22.9\end{cases}
$$

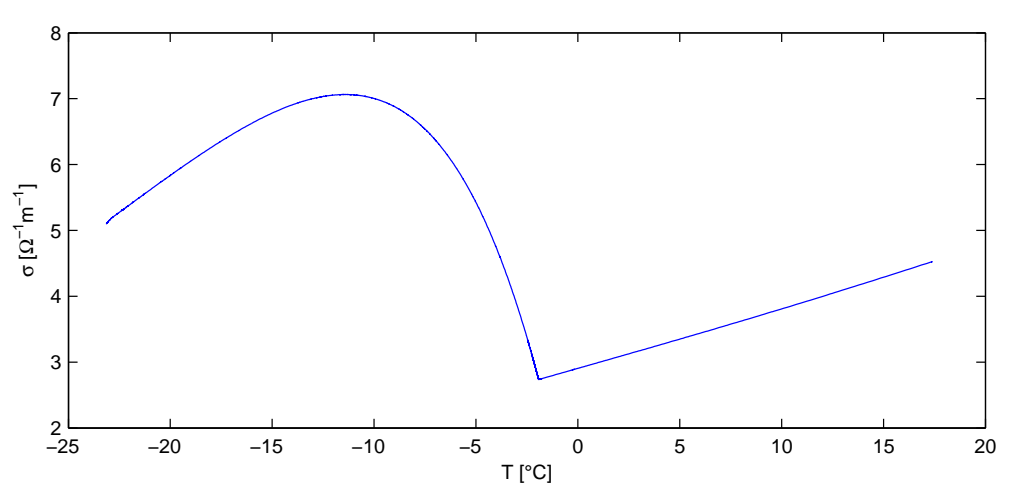

Figure 2.2: The conductivity of sea water and then brine in sea ice. 
The figure shows an increase in conductivity between $-2^{\circ} \mathrm{C}$ and $12^{\circ} \mathrm{C}$. This is due to an increase in salinity as ice begins to form. In other temperature regions, the conductivity decreases with decreasing temperature as the ion mobility decreases. Brine conductivity is largely frequency independent.

Brine exhibits a Debye relaxation at $\mathrm{GHz}$ frequencies. This is depicted in Figure 2.4. This is well beyond the range of our measurements and we measure the temperature and salinity dependent low frequency permittivity $\epsilon_{D C}$.

$$
\epsilon_{D C}=\chi+\epsilon_{\infty} .
$$

Stogryn and Desargant (1985) present the following expression for $\epsilon_{D C}$ :

$$
\epsilon_{D C}=\frac{939.66-19.068 T}{10.737-T} \text {. }
$$

This dependence is shown in Figure 2.3. We do not measure dielectric loss in the experimental frequency range.

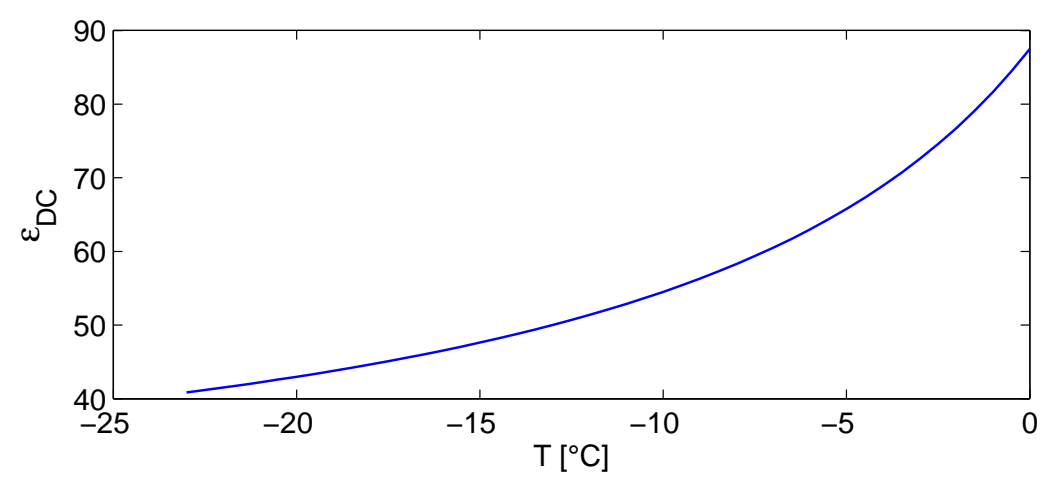

Figure 2.3: The low frequency permittivity of brine in sea ice. 

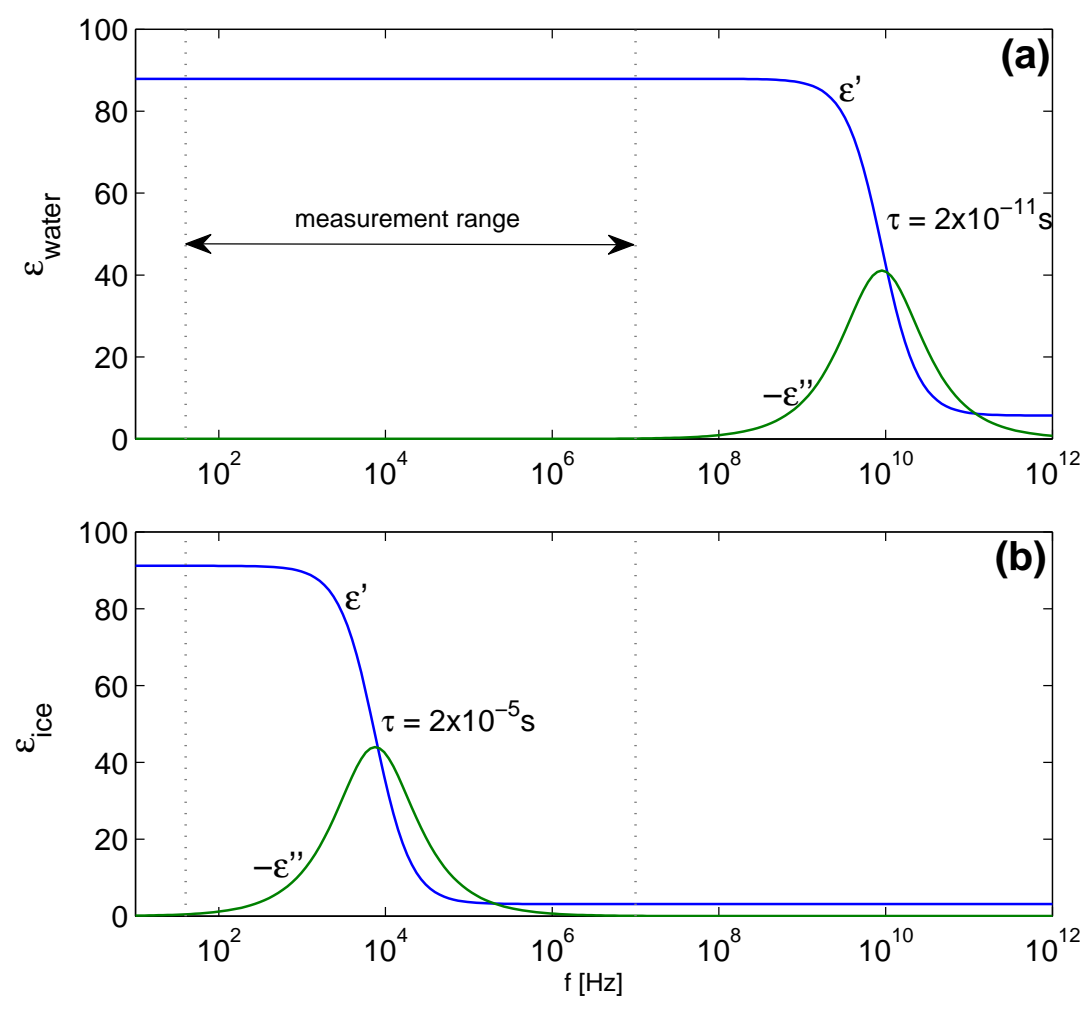

Figure 2.4: Dielectric relaxations of brine (a) and ice (b) at $-20^{\circ} \mathrm{C}$. 


\subsection{The electrical properties of ice}

The assembly of $\mathrm{H}_{2} \mathrm{O}$ molecules into a crystalline ice lattice is governed by two selection rules. These are that:

- there are two hydrogen's adjacent to each oxygen, and

- there is only one hydrogen per bond.

Such an arrangement does not facilitate electrical conduction and, in reality, the ice rules are violated at certain locations within the crystal. There are orientational (Bjerrum) and ionic defects which can progress through the lattice [Jaccard (1959)]. Both defect types include positive and negative charge carriers. The processes require reorientation of molecules or reordering of bonds. Intrinsic ice is not a good electrical conductor due to a low abundance of point defects, however, it is susceptible to doping. Inclusions of impurities within the ice matrix increases the concentration of defects. This drastically increases the conductivity of the sample. For this reason, ice is some times described as a protonic semiconductor. Like electronic semiconductors, the electrical conductivity increases with temperature. This sensitivity to impurities, and the occurrence of surface conduction (the defects are concentrated at the crystal boundaries), means that the conductivity of a pure ice crystal is ill defined.

The ice portion in sea ice is polycrystalline, fractured, and contains impurities. The variation of the conductivity with temperature can not be generalised like Stogryn and Desargant (1985) were able to do with brine.

Ice becomes polarized by reorientation of molecules or bonds. They slip into a low energy state and produce a dipole moment aligned with the polarizing field. This process requires some thermal activation energy $E_{a}$, in order to overcome the potential confinement of lattice neighbours and to violate local lattice laws. For this reason, polarization is a slow mechanism in ice and relaxation occurs at a low frequency. Figure 2.4 shows this. The main difference between the dielectric properties of water 


\begin{tabular}{|c|c|c|c|}
\hline & pure & $\mathrm{NaCl}$ doped $(0.01 \mathrm{M})$ & $\mathrm{NaCl}$ doped $(0.1 \mathrm{M})$ \\
\hline$\chi$ & 99 & not given & not given \\
\hline$\tau$ & $1.8 \times 10^{-4} \mathrm{~s}$ & $3 \times 10^{-5} \mathrm{~s}$ & $1 \times 10^{-5} \mathrm{~s}$ \\
\hline
\end{tabular}

Table 2.1: Polarization parameters for pure and doped ice at $-20^{\circ} \mathrm{C}$.

and ice is the time constant of their relaxation. We observe the relaxation of ice in the middle of our measurement range whereas only the low frequency polarization of water is observed.

The relaxation time of ice follows a Boltzmann distribution:

$$
\tau \propto \exp \frac{E_{a}}{k_{B} T}
$$

where $T$ is temperature $[\mathrm{K}], k_{B}$ is the Boltzmann constant, and $E_{a}$ is the activation energy of the low frequency polarization mechanism. The relaxation time is strongly dependent on impurity content. Table 2.1 summarises experimental results for polycrystalline pure ice samples [Johari and Whalley (1981)] and $\mathrm{NaCl}$ doped ice [Grimm et al. (2008)] at $20^{\circ} \mathrm{C}$.

The susceptibility of the ice relaxation is also temperature dependent. For a 'pure ice' sample, Evans (1965) gives:

$$
\epsilon_{D C}=\frac{20715}{T+273-38}
$$

and a temperature independent high frequency permittivity $\epsilon_{\infty}=3.14$. Values of the susceptibility of $\mathrm{NaCl}$ doped ice are absent in the literature. For $\mathrm{HCl}$ doped ice , Takei and Maeno (1987) show that, at high temperatures the susceptibility is similar to that of pure ice. 


\subsection{Multiphase dielectric mixture theory}

The goal of dielectric mixture theory is to represent the electrical response of a multiphase system with effective parameters $\epsilon_{e f f}$ and $\sigma_{\text {eff }}$. These parameters depend on the phase geometry and the electrical properties $\left(\epsilon_{i}\right.$ and $\sigma_{i}$ ) of the respective phases.

Maxwell (1873) was the first to consider the effective electrical properties of a composite medium. He showed that a series network of conductive dielectrics undergoes a dielectric relaxation, even if the electrical properties of the constituents are independent of frequency. Wagner (1914) extended the theory to a composite of dispersed spheroids and then Sillars (1937) to ideal dielectric ellipsoids aligned with a polarizing field. A similar frequency dependence resulted. The term 'Maxwell/Wagner/Sillars dispersion' or 'Maxwell/Wagner/Sillars polarization' describes the phenomenon which causes materials to exhibit dispersion even though their constituent components are independent of frequency.

Addison (1970) was the first to apply the theory to explain the electrical response of sea ice. He interpreted a high frequency $(\sim 10 \mathrm{MHz})$ relaxation observed in-terms of Sillar's ellipsoids and concluded that his experimental result was compatible with a sea ice microstructure in which one quarter of the brine is included within ellipsoids with an aspect ratio of 50 .

Tinga et al. (1973) formulated a more useful model for the complex conductivity of dispersed ellipsoids which includes the first order effect of particle interaction. This makes it more applicable at high brine volume fractions. It includes an input parameter which sets the orientation of the ellipsoids with respect to the polarizing field. For ellipsoids with long axis $a$ and short axis $b=c$, and eccentricity $e=\left[1-\left(\frac{b}{a}\right)^{2}\right]^{\frac{1}{2}}$, the effective permittivity is:

$$
\epsilon_{e f f}^{*}=\epsilon_{1}^{*}\left(1+\frac{V_{2}}{V_{1}} \frac{\epsilon_{2}^{*}-\epsilon_{1}^{*}}{\epsilon_{1}^{*}+n\left(\epsilon_{2}^{*}-\epsilon_{1}^{*}\right)\left(1-V_{2} / V_{1}\right)}\right)
$$


where $\epsilon_{2}^{*}$ is the complex permittivity of the dispersed phase, $\epsilon_{1}^{*}$ the complex permittivity of the background phase, $\frac{V_{2}}{V_{1}}$ is the volume fraction of the dispersed phase, and $n$ is the depolarization ratio given by:

$$
\begin{aligned}
n^{b, c} & =\left(\frac{b}{a}\right)^{2}\left(\frac{1}{4 e^{3}}\right)\left(\frac{2 e}{(b / a)^{2}}+\ln \frac{1-e}{1+e}\right) \\
n^{a} & =\left(\frac{b}{a}\right)^{2}\left(\frac{1}{2 e^{3}}\right)\left(-2 e+\ln \frac{1+e}{1-e}\right)
\end{aligned}
$$

for electric fields orientated along the short axis, or long axis respectively. Tinga et al. (1973) successfully used the model to explain the observed large permittivities of wet wood at $\mathrm{GHz}$ frequencies.

Vant et al. (1978) applied the model of Tinga et al. (1973) to experimental sea ice permittivities at $\mathrm{GHz}$ frequencies. They considered the dispersed ellipsoids of the model to be applicable to the elongated brine pocket microstructure which can exist in sea ice. The model was adapted slightly to better represent sea ice microstructure. It described the effective permittivity of ellipsoids which lie at an angle $\theta$ from the vertical but with a random azimuthal orientation. Then, assuming a pocket aspect ratio, and for a known brine volume fraction, they attempted recovery of brine pocket orientation from inversion of the above equations. Experimental results for the permittivity of pure ice and a $\mathrm{NaCl}$ solution were used for $\epsilon_{1}^{*}$ and $\epsilon_{2}^{*}$ respectively.

More recently, Stogryn and Desargant (1985) published their work on the dielectric and conductive properties of brine in sea ice. Their expressions, summarised in the previous sections, serve as a better inputs for $\epsilon_{2}^{*}$. This new model has been coined the 'VTS model' (Vant-Tinga-Stogryn). It has been applied by Backstrom and Eicken (2006) and Pringle et al. (2009) to model artificial, and field, $50 \mathrm{MHz}$ sea ice permittivity measurements. 


\subsection{Space charge polarization}

A boundary between regions of contrasting charge transfer mechanisms blocks the flow of current. Under application of a steady electric field, free charge piles up at the interface producing an electric double layer. This is a distribution of charge which satisfies an equilibrium between concentration diffusion and a Lorentz force. For a one-dimensional charge distribution with stable carrier populations the following differential equation holds [Macdonald (1953)]:

$$
\frac{\partial^{2} n}{\partial x^{2}}=\mu \frac{\partial(n E)}{\partial x}
$$

where $x$ is the spatial coordinate, $n$ is the charge carrier concentration and $E$ is the electric field due to all the charges in the distribution. A large potential drop occurs across the charge distribution. Beyond the electric double layer, the medium is mostly screened from the polarizing field. The distribution length is characterised by the Debye length:

$$
\lambda_{D}=\sqrt{\frac{k_{b} \mathrm{~T} \epsilon^{\prime} \epsilon_{0}}{2 e^{2} n}}
$$

where $k_{B}$ is the Boltzmann constant and $e$ is the elementary charge. The Debye length depends on the temperature $T$, and charge carrier concentration $n$ and the permittivity $\epsilon^{\prime}$ of the material. The greater the charge carrier concentration, the steeper the charge distribution and the larger the space charge polarization.

The theory of space charge polarization is quite well developed for the case where the interface is completely blocking. When no boundary charge transfer occurs, the space charge distribution constitutes a double layer capacitance of magnitude [Barsoukov and Macdonald (2005)]:

$$
\begin{aligned}
C_{D L} & =\sqrt{\frac{2 e^{2} n \epsilon^{\prime} \epsilon_{0}}{k_{b} T}} \cosh \left(\frac{e V_{0}}{2 k_{b} T}\right) \\
& \approx \frac{\epsilon^{\prime} \epsilon_{0}}{\lambda_{D}}
\end{aligned}
$$


where $V_{0}$ is the potential across the interface. The approximation holds at sea ice temperatures.

Three charge transfer mechanisms feature in sea ice impedance measurements. These are:

- electronic conduction in the metal electrodes,

- protonic conduction in the ice portion, and

- ionic conduction in the brine.

Space charge polarization occurs at every interface between these regions. It is illuminating to calculate the Debye length for brine and ice. In pure ice the concentration of Bjerium defects (the majority charge carriers) is given by Boltzmann statistics as $3 \times 10^{18} \mathrm{~m}^{-3}$ at $-20^{\circ} \mathrm{C}$. Substituting this into the above equations yields $\lambda_{D}=2 \times 10^{-6}[\mathrm{~m}]$ and $C_{D L}=0.4\left[\mathrm{mF} / \mathrm{m}^{2}\right]$. Sea ice brine at $-20^{\circ} \mathrm{C}$ has an ionic concentration of $1 \times 10^{26}\left[\mathrm{~m}^{-3}\right]$, a Debye length of $4 \times 10^{-10}[\mathrm{~m}]$ and $C_{D l}=2\left[\mathrm{~F} / \mathrm{m}^{2}\right]$. The Debye length of brine is many orders of magnitude smaller than for ice indicating that the $\mathrm{Na}^{+}$and $\mathrm{Cl}^{-}$ ions in the brine phase dominate space charge interfacial polarization. The Debye lengths are also much smaller than the microstructure dimensions of sea ice suggesting that, for this composite, it might be appropriate to treat the space charge distribution as a surface charge distribution.

Space charge polarization contributes at low frequencies. It requires the migration of ions to form the electric double layer and is therefore limited by the ion mobility. AC theories of space charge polarization are complex and not particularly enlightening and will not be considered here. 


\section{Chapter 3}

\section{Lab freezer experiment}

This chapter documents an attempt to recreate the Antarctic experiment in a laboratory chest freezer. We attempted to grow a small sample of columnar sea ice and monitor its anisotropic impedance throughout its growth. The impedance sensors were parallel plate capacitors, identical to those deployed in Antarctica.

This experiment was overly ambitious, and ultimately superseded. However, at the time it was performed, it was extremely illuminating to the author. It introduced the experimental procedure, the instrumentation and equipment used, and the type of impedances and permittivities which could be expected. It forewarned of the difficulty in gaining a physical insight into the low frequency electrical properties of sea ice. Following its completion several questions and challenges became apparent. From this point, the project was steered towards answering and overcoming these. It is logical to describe the experiment in full at this point of the thesis to give the reader the same insight. 


\subsection{Experimental}

\subsubsection{Ice growth}

The sea ice sample was grown in a plastic container of dimensions $32 \mathrm{~cm}(\mathrm{~L})$ $\times 21 \mathrm{~cm}(\mathrm{~W}) \times 18 \mathrm{~cm}(\mathrm{H})$. The container was lined with polyurethane foam, approximately $5 \mathrm{~cm}$ thick around its sides and underneath. This insulation would diminish heat loss through these surfaces and heat loss through the open top of the container would be the dominant process. This would force the one-dimensional ice growth which occurs in natural sea ice formation. We hoped that this would produce a columnar sea ice microstructure and anisotropic electrical properties.

The container was filled with approximately $12 \mathrm{~L}$ of sea water sourced at the local Island Bay beach. It was then enclosed in a 500L chest freezer with air temperature maintained at approximately $-21^{\circ} \mathrm{C}$.

The temperature profile within the container was monitored by seven thermistors. Four of these were positioned along a central vertical axis and three on a central horizontal plane. This configuration would demonstrate whether one-dimensional cooling was achieved. The thermistors were electrically insulated using heat shrink and RTV silicon. They formed a potential divider with a known resistance. This network was maintained at a constant potential, and by measuring the thermistor voltage drop, the thermistor resistance was calculated. A National Instruments PCI data logger (PCI-6221) measured this potential drop. High precision thermistors, identical to those of Pringle (2004), were used. The temperature was inverted from the measured resistance using the callibration expressions of Pringle (2004). We estimate a temperature measurement accuracy of $\Delta T=0.1^{\circ} \mathrm{C}$ based on the observed variance in measured temperature using identical thermistors deployed in a water bath. 


\subsubsection{Impedance measurement cells and instrumentation}

The measurement cells were circular, parallel plate capacitors identical to those deployed in Antarctica. The plates were 1mm thick circular discs, of radius $45 \mathrm{~mm}$ and constructed of marine grade stainless steel. The plates were held $25 \mathrm{~mm}$ apart by two plastic bars. They were mounted in horizontal and vertical orientations, side by side, within the experimental container. Their position is shown in Figure 3.2.

Impedance measurements were performed using an Agilent 4294a impedance analyser. This instrument functions by operating an autobalancing bridge and is capable of four-terminal, precision impedance measurements across the frequency range $40 \mathrm{~Hz}-110 \mathrm{MHz}$. We intended to use as much of this range as possible. A four-terminal measurement is depicted in Figure 3.1. The $\mathrm{H}_{c}$ and $\mathrm{L}_{c}$ terminals inject and draw current into the load. The potential is measured across terminals $\mathrm{H}_{p}$ and $\mathrm{L}_{p}$. In theory, because the potential terminals draw no current, this technique is insensitive to the impedance of the measurement cables. In reality, any extension of the cables beyond the calibration plane introduces a measurement error. This error can be reduced by performing open/short/load compensation as described in Appendix A.

Custom measurement cables were fabricated to connect the instrument to the measurement cells. The cables were coaxial and they were utilised in a four-terminal pair (4TP) configuration. In this configuration, depicted in Figure 3.1, one current and one voltage cable connects independently to each electrode. The coaxial shielding terminates as near as possible to the cell. Agilent Technologies (2009) advises this configuration is suitable for measuring a large impedance range $(1 \mathrm{~m} \Omega-10 \mathrm{M} \Omega)$ from low to high frequencies.

Four $1 \mathrm{~m}$ RG-58C/U coaxial cables were connected to the vertical capacitor and four $1.5 \mathrm{~m}$ cables were connected to the horizontal capacitor. For both cable sets, the coax shielding terminated communally $\sim 3 \mathrm{~cm}$ from the electrodes. This point was electrically insulated, using heat shrink, hot 


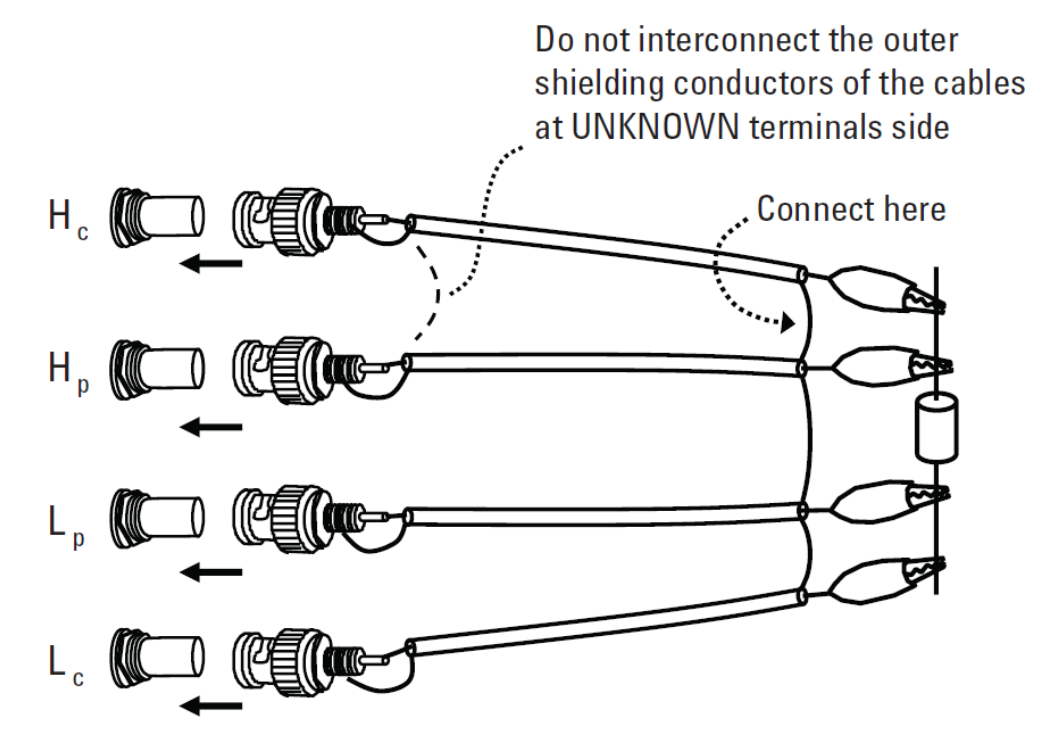

Figure 3.1: Four-terminal pair cable configuration (reproduced from Agilent Technologies (2009)).

glue and RTV silicon. Four insulated wires extended to the cell electrodes. They paired into twos $\left(\mathrm{H}_{p}, \mathrm{H}_{c} \& \mathrm{~L}_{p}, \mathrm{~L}_{c}\right)$, and were crimp fitted with a ring connector which bolted directly to each electrode. Male BNC connectors were fitted at the instrument end of the cables.

Open, short, and load measurements were performed to characterise both sets of measurement cables and to allow for post-measurement open/short/load compensation. These conditions were imposed at the cable end terminals (where the ring connectors would attach to the electrodes) and the impedance was measured across the entire circuit (cables + load). The open circuit measurement was made with cable end terminals separated by the same distance as they are when connected to the capacitor. For the short circuit measurement the terminals were bolted together. The standard reference load was an RC series circuit consisting of a $560 \Omega$ standard ceramic resistor and a 200nF electrolytic capacitor. This load was chosen in an attempt to replicate the impedance that sea ice 


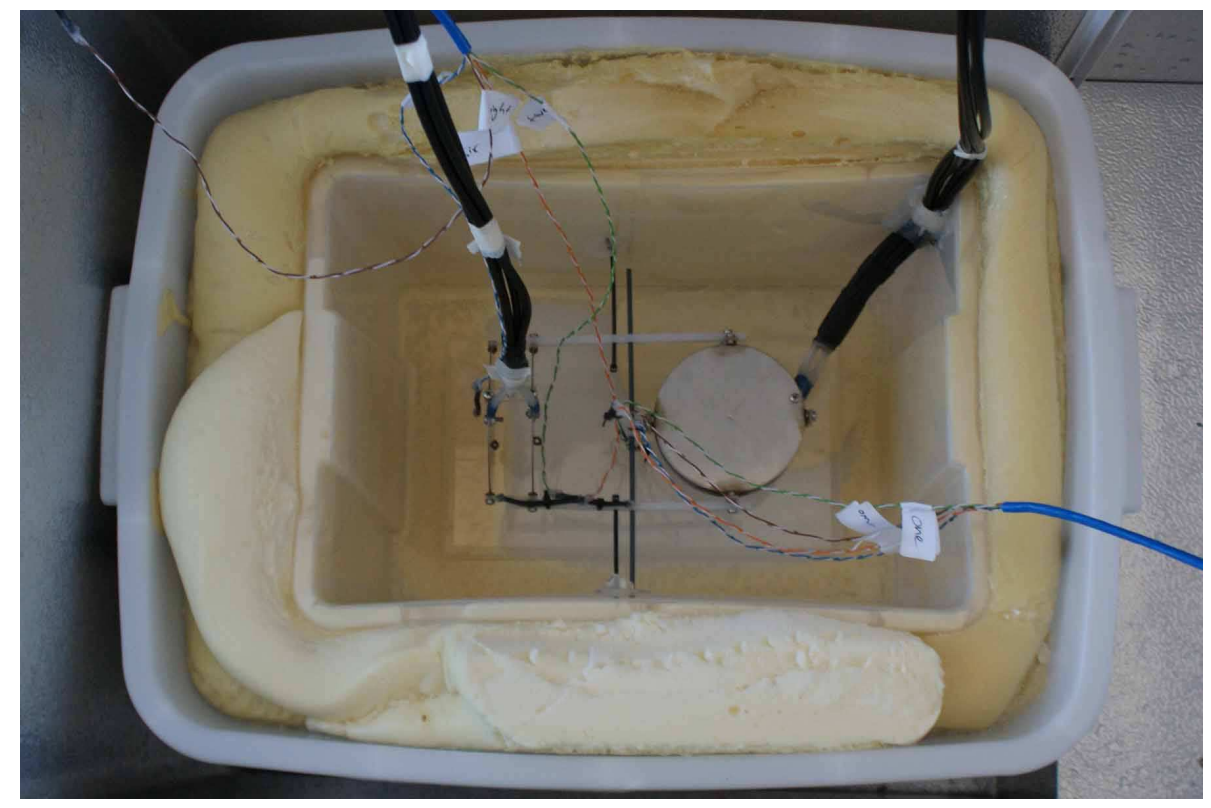

Figure 3.2: The experiment sitting within the chest freezer. Vertical and horizontal capacitors are pictured on the left and right of the tub respectively. The black cables are the coaxial measurement cables and the striped cables connect to the thermistors.

would produce. The true impedance of the reference load was measured without error using an Agilent 16047E front panel test fixture mount. Open/short compensation was performed on this test fixture to negate its residuals. The impedance of the capacitors in air was also measured so that the permittivity could be calculated.

The cables extended from the measurement cells in the freezer to the bench top impedance analyser. Impedance measurements were made at 14 time intervals over the duration of the experiment. The instrument settings are summarised in Table 3.1.

These settings configured an impedance measurement sweep between $40 \mathrm{~Hz}$ and $110 \mathrm{MHz}$. The impedance was measured at 201 logarithmically spaced frequencies across this range. The bandwidth setting maximised the device resolution and six sweeps were averaged to reduce noise. The 


\begin{tabular}{|l|r|}
\hline Start frequency & $40 \mathrm{~Hz}$ \\
\hline Stop frequency & $110 \mathrm{MHz}$ \\
\hline Frequency scale & logarithmic \\
\hline Measurement points & 201 \\
\hline Bandwidth & 5 \\
\hline Averaging factor & $\times 6$ \\
\hline $\mathrm{V}_{\text {osc }}$ & $500 \mathrm{mV}$ \\
\hline
\end{tabular}

Table 3.1: Impedance analyser settings. Note that the bandwidth setting is a device setting which maximises the measurement accuracy. It is not $5 \mathrm{~Hz}$.

load was driven with a $500 \mathrm{mV}_{R M S}$ sinusoidal signal. 


\subsection{Results and analysis}

\subsubsection{Evaluation of ice grown}

The sea ice produced had a slushy consistency and, for most of the experiment, a finger could be pushed into it. A puddle of brine also pooled at the top of the container. This frozen sea water was a poor reproduction of natural sea ice. It had an unrealistically high brine volume fraction and salinity due to the size constraints of the container. The volume expansion as water turned to ice forced the brine pool to the top of the container.

The temperature data shown in Figure 3.3 indicates that one-dimensional cooling was achieved. Figure 3.3(a) shows temperature data for a vertical string of thermistors. The string was positioned at the horizontal centre of the container. The legend gives the position of the thermistors below the water level. The plot shows a vertical temperature gradient with heat loss directed out the open top of the container. Figure 3.3(b) shows thermistors placed in a horizontal plane at a depth of $8 \mathrm{~cm}$ below the initial water level. The data shows a uniform temperature variation. The temperature profile was $T(x, y, z)=T(z)$ where $z$ is the vertical axis. The foam insulation regulated heat loss sufficiently to achieve one-dimensional cooling with heat loss directed out the top of the container.

It took approximately one week for the sea ice to form and cool to the freezer air temperature of $-21^{\circ} \mathrm{C}$. The vertical temperature gradient was at times quite steep. At 60 hours the temperature varied by approximately $3^{\circ} \mathrm{C}$ across the $9 \mathrm{~cm}$ diameter vertical capacitor. This gradient was much larger than that which would be encountered in natural sea ice. For simplicity, we assumed that the temperature was uniform across both cells and we used the central temperature to characterise the state of the ice in subsequent analysis. 

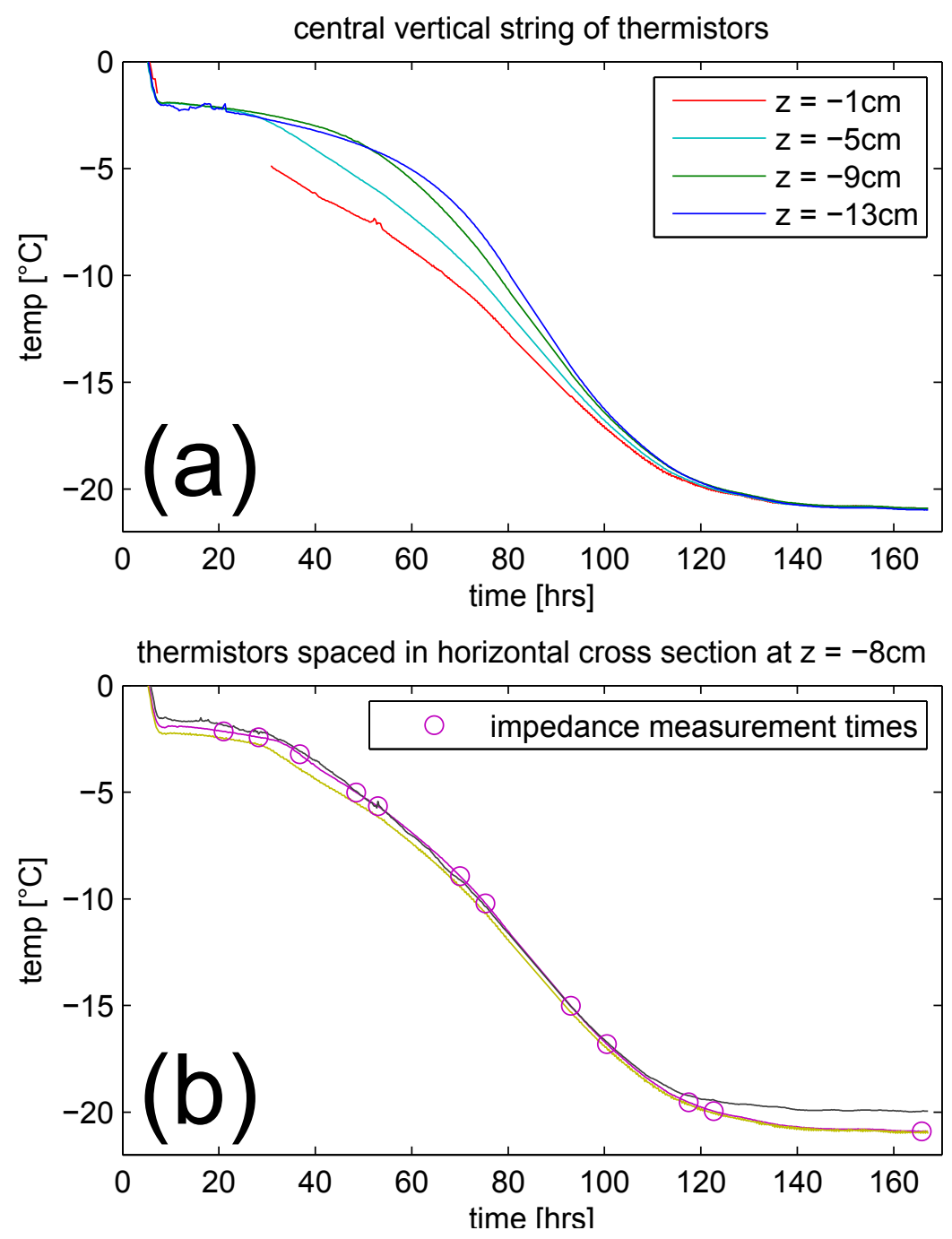

Figure 3.3: Temperature profiles along a vertical profile and a horizontal plane. $\mathrm{z}$ is the distance of the thermistors below the initial water level. 


\subsubsection{Impedance data}

Impedance measurements were made at 14 time intervals over roughly a weeks duration. The timing was slightly irregular as the measurements were made around a working day. The first two measurements were made before ice formation had begun. The circles on Figure 3.3(b) mark the final 12 impedance measurement occasions.

Figure 3.4 displays the open/short/load compensated impedance data as measured by horizontal and vertical capacitors. The data are presented as horizontal and vertical components of the sea ice impedance. A representative sample of the temperature variation is shown. The quoted temperatures refer to those at the centre of the container.

The plot shows that the sea ice impedance increased as the temperature was reduced. The vertical component of the impedance was approximately a factor of two times larger than the horizontal component across the measurement frequency range. This anisotropy is not consistent with the columnar microstructure we attempted to produce. It was caused by the pool of brine at the top of the container which the vertical capacitor extended further in to. 

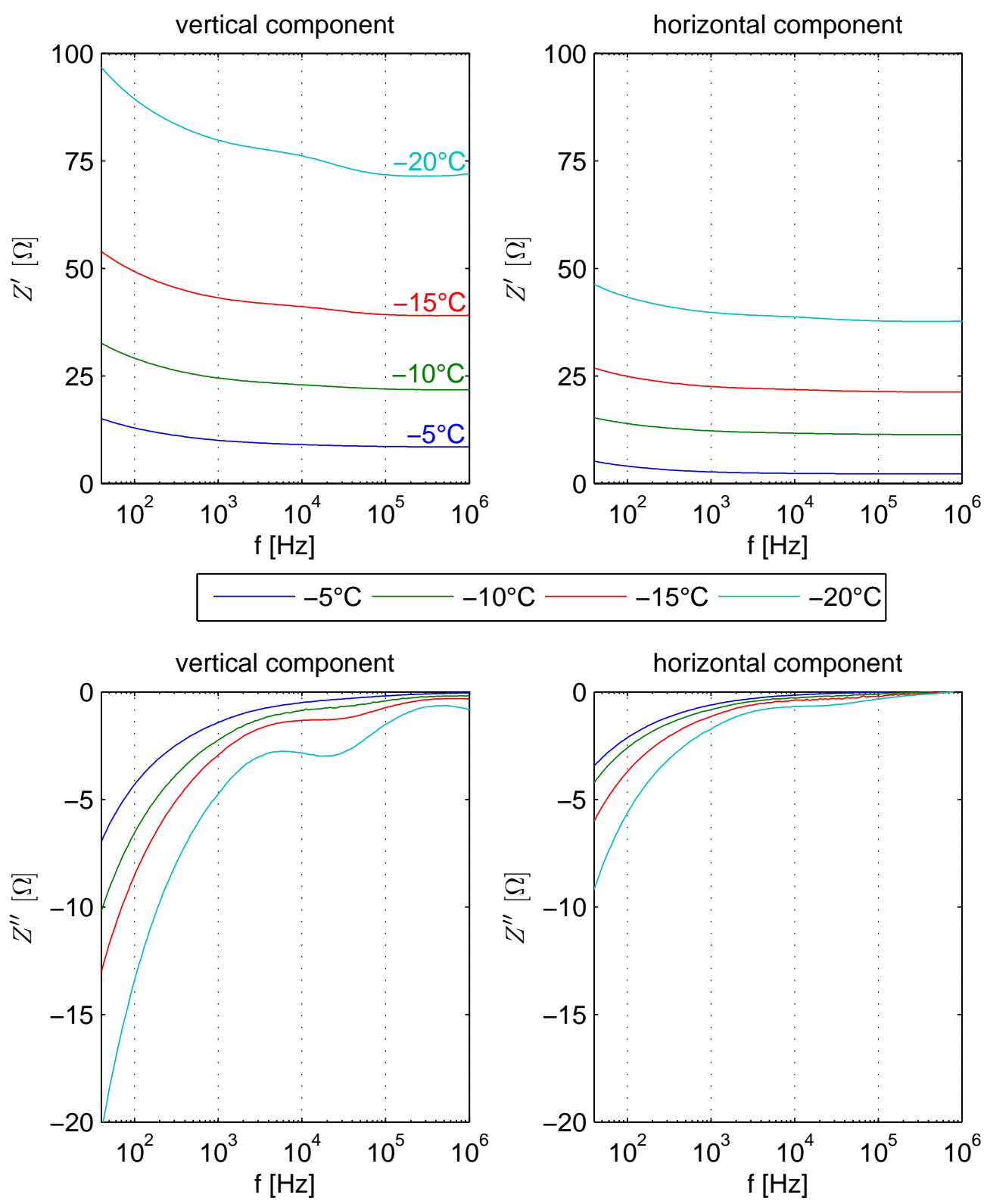

Figure 3.4: The horizontal and vertical components of the broadband impedance of laboratory grown sea ice. 


\subsubsection{Impedance measurement error}

The open/short/load compensation technique was evaluated by examining the corrected sea ice impedance data. The imaginary component of the impedance should remain negative at high frequencies (as the real permittivity is positive). If it becomes positive then this indicates an impedance measurement error.

Figure 3.5 presents the results of the compensation for three sea ice measurements. The measurements were made across the horizontally orientated capacitor which has $1.5 \mathrm{~m}$ long measurement cables. Impedances corrected using the open/short/load technique are shown alongside the raw impedance data. Note that the $y$-axis of the imaginary impedance plot is negative and logarithmic to excentuate the frequency where the correction methods fails. The plot shows that, for low impedance sea water,

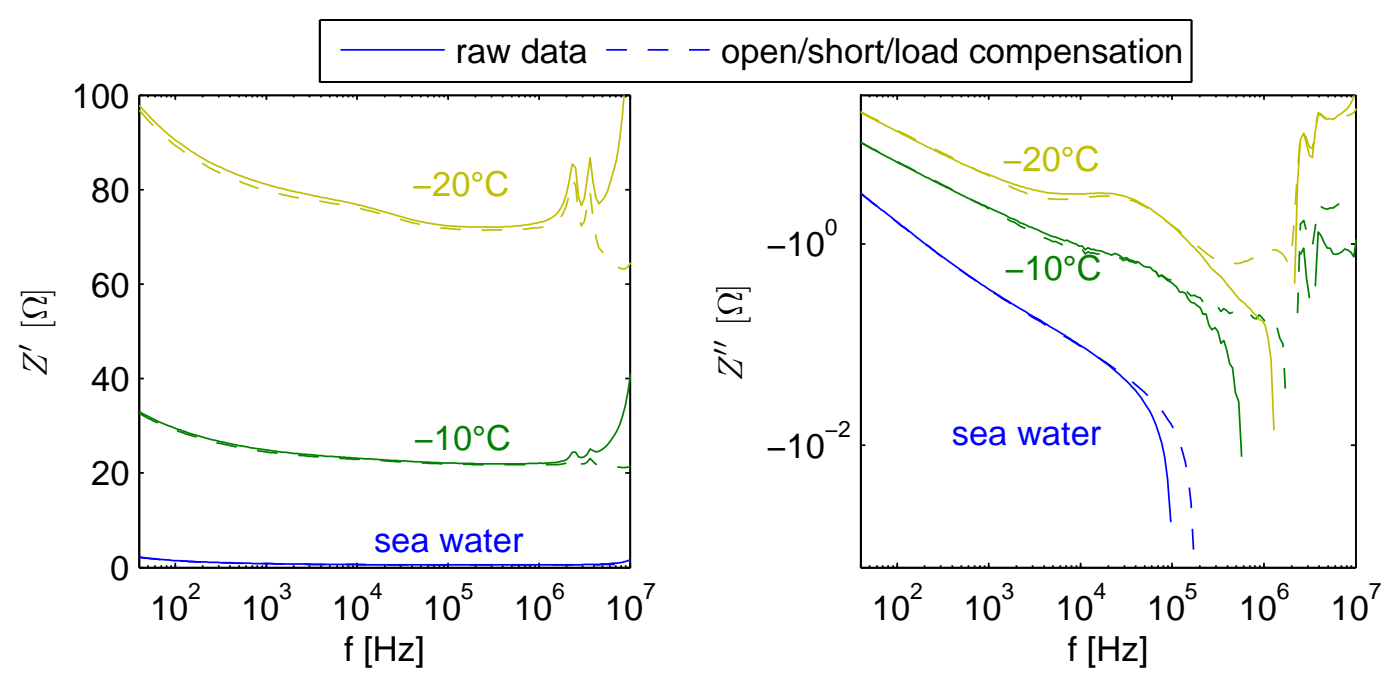

Figure 3.5: Comparison of open/short/load compensated impedance with raw data for the horizontal capacitor.

the imaginary raw impedance became erroneous at $100 \mathrm{kHz}$. Performing open/short/load compensation increased the useful frequency range to $200 \mathrm{kHz}$. The compensated reactance of $-10^{\circ} \mathrm{C}$ and $-20^{\circ} \mathrm{C}$ sea ice remained 
accurate up to approximately $1 \mathrm{MHz}$. The data showed that the frequency where the correction method failed depended on the load impedance. The measured resistance was less prone to error and open/short/load compensation produced little change below $1 \mathrm{MHz}$. We present sea ice impedance measurements up to a frequency of $1 \mathrm{MHz}$ and acknowledge the imaginary component may be erroneous above $200 \mathrm{kHz}$. The resistance is accurate across this entire range.

\subsubsection{Permittivity data}

The relationship between the measured impedance $Z$ and the complex permittivity $\epsilon^{*}$ is:

$$
Z=\frac{1}{j \omega C_{0} \epsilon^{*}}
$$

where $C_{0}$ is the air capacitance of the cell. We measured $Z_{0}$, the impedance of the capacitor in air, where:

$$
Z_{0}=\frac{1}{j \omega C_{0}}
$$

and recovered the relative complex permittivity using the relationship:

$$
\epsilon^{*}=\frac{Z_{0}}{Z}
$$

Figure (3.6) shows the real and imaginary components of the complex permittivity. The permittivity data spans an enormous range and a log log scale is necessary to display it.

The imaginary component of the permittivity demonstrated a strong $1 / \omega$ dependence and showed no evidence of dielectric relaxation. This is the same dependence that Addison (1969) observed and reflects that, in sea ice, the conductive loss $\left(\sigma_{D C} / \omega\right)$ is much larger than the dielectric loss.

The real component of the permittivity showed a similar frequency dependence. The magnitude of the low frequency permittivity is remarkable in comparison to the dielectric constant of both water and ice 

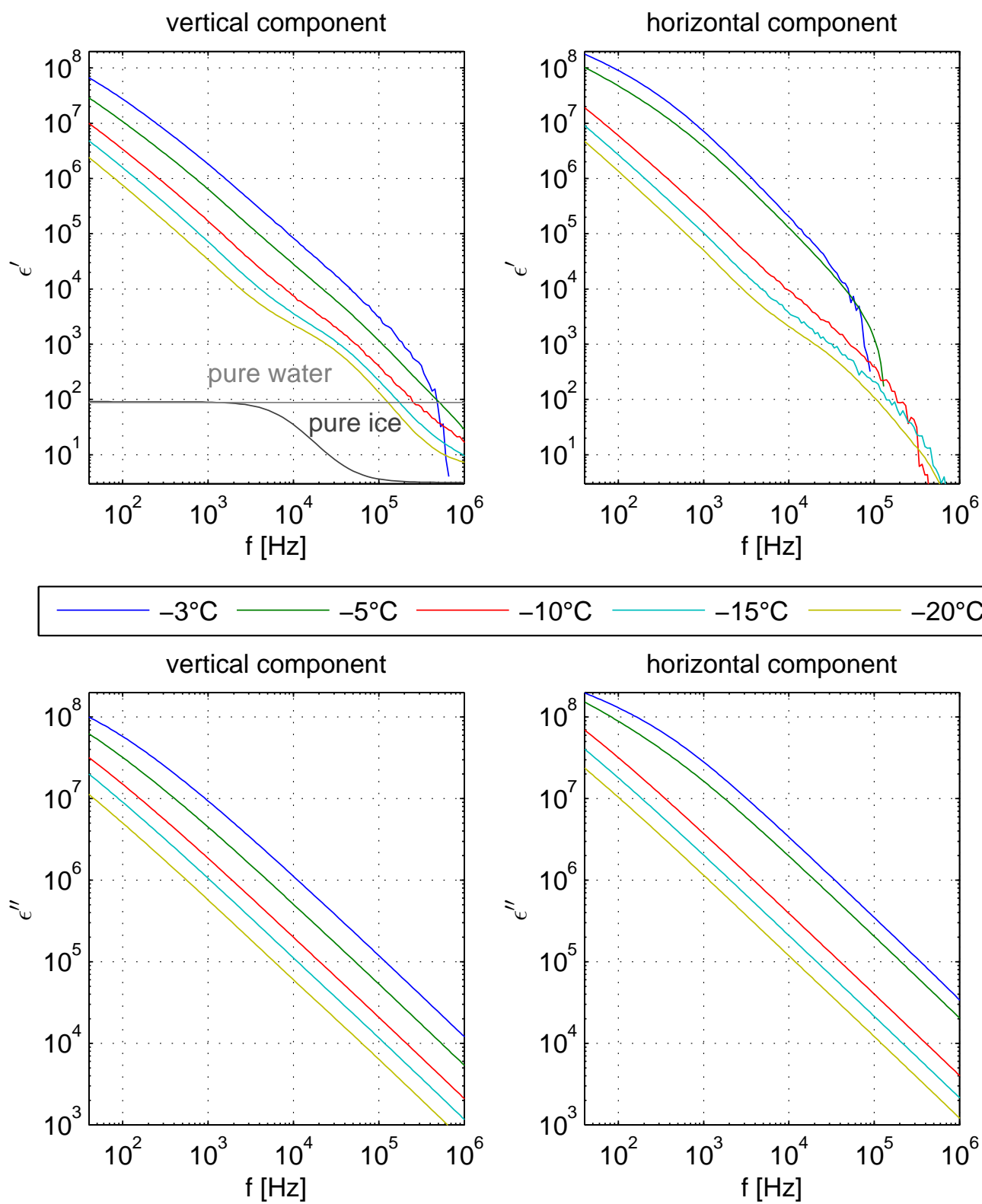

$-10^{\circ} \mathrm{C}--15^{\circ} \mathrm{C}-20^{\circ} \mathrm{C}$

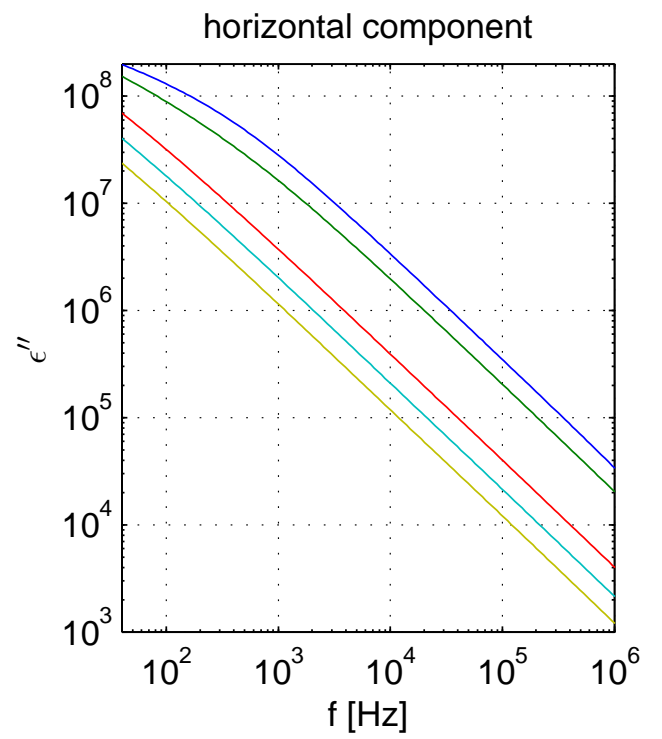

Figure 3.6: The horizontal and vertical components of the complex broadband permittivity of laboratory sea ice. The dielectric constant for pure water \& ice at $0^{\circ} \mathrm{C}$ is also shown [Evans (1965); Lide (1993)]. 
$(\sim 100)$. Evidently, the response is not due to molecular polarization of $\mathrm{H}_{2} \mathrm{O}$ in either its solid or liquid form. The massive low frequency permittivity is due overwhelmingly to ionic polarization (space charge polarization). This occurs at interfaces within the material that block the flow of current. This includes ice/brine and brine/electrode interfaces. The latter is electrode polarization. Space charge polarization is an inherently low frequency process and, at microwave frequencies, the permittivity should flatten off between approximately 3-100 depending on the relative proportions of ice and brine and the dispersed phase geometry. Many experiments performed in this high frequency range have confirmed this [Addison (1969); Vant et al. (1978)]. The vertical component of the permittivity drops below 100 above $100 \mathrm{kHz}$ and begins to flatten off at $1 \mathrm{MHz}$. This suggests that space charge polarization is the dominant polarization process up until 100kHz. 


\subsection{Discussion}

The container of frozen sea water produced was not a good reproduction of natural sea ice. Nonetheless, this experiment played a crucial role in the development of this thesis. Its usefulness was largely, gaining familiarity with the range and magnitude of impedances that could be expected, as well as demonstrating the experimental difficulties in obtaining them. It showed that interpreting the permittivity data was complicated and forewarned that the recovery of microstructure data would prove difficult. The thesis was at this point steered towards answering a number of questions, or resolving difficulties, which arose from this experiment. These were:

- realising the importance of electrode polarization,

- obtaining accurate high frequency impedance data,

- investigating a better way to visualise data to aid physical interpretation, and

- producing more realistic sea ice and non-invasive measurement cells.

The remainder of this chapter is devoted to summarising what was learnt about these points and outlining the direction forward.

\subsubsection{Electrode polarization}

The first impedance measurement of the experiment was performed when the electrodes were immersed in room temperature sea water and the corresponding permittivity was enormous $\left(\epsilon^{\prime}=1.6 \times 10^{8}\right.$ at $\left.100 \mathrm{~Hz}\right)$. It was demonstrated that this was due to electrode polarization by moving the electrodes closer together, and observing that the measured reactance did not change. In contrast, the measured resistance scaled with electrode separation. 
This experiment can be understood by representing the total cell impedance as a series combination of electrode impedances $\left(Z_{e}\right)$, and a single impedance representing the sample bulk $\left(Z_{\text {sample }}\right)$.

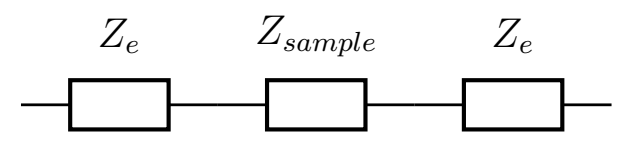

For a cylindrical sample with face area $A$, length $d$, and complex permittivity $\epsilon^{*}$, the impedance of the entire cell (sample + electrodes) is:

$$
Z_{\text {cell }}=2 Z_{e}+\frac{d}{j \omega \epsilon_{0} A \epsilon^{*}} .
$$

The electrode impedance is due to electrode polarization and can be represented by a capacitive circuit element $C$. The equivalent circuit becomes:

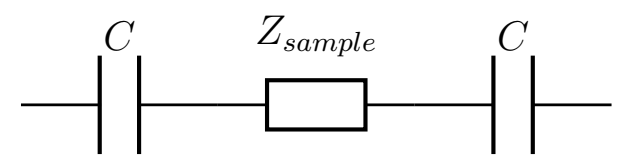

For uniformly polarized electrodes, the cell impedance is:

$$
\begin{aligned}
Z_{\text {cell }} & =\frac{2}{j \omega A C_{D L}}+\frac{d}{j \omega \epsilon_{0} A \epsilon^{*}} \\
& =\frac{1}{j \omega A}\left(\frac{2}{C_{D L}}+\frac{d}{\epsilon_{0} \epsilon^{*}}\right)
\end{aligned}
$$

where $\mathrm{C}_{D L}$ is the double layer capacitance per unit area.

The second term in the parentheses in equation 3.5 scales with sample length. The first term does not. The dominance of electrode polarization in an impedance measurement depends on the relative magnitude of the imaginary component of these two terms - it depends on the sample length $(d)$, the material properties $\left(\epsilon^{\prime}, \epsilon^{\prime \prime}\right)$ and the interaction of the sample 
with the charged electrode $\left(C_{D L}\right)$. The condition under which electrode polarization dominates the measurement is:

$$
\frac{2}{C_{D L}}>>\frac{d \epsilon^{\prime}}{\epsilon_{0}\left(\epsilon^{\prime 2}+\epsilon^{\prime \prime 2}\right)} \text {. }
$$

For the sea water, varied electrode spacing experiment described above, this condition was satisfied. The electrical properties of brine and sea water were summarised in section 2.2. An estimate of the double layer capacitance of brine was made in section 2.5. These inputs satisfy equation 3.6 giving the order of magnitude comparison of $1>>10^{-7}\left[\mathrm{~m}^{2} / \mathrm{F}\right]$ at $100 \mathrm{~Hz}$. Both this calculation, and the experimental observation, confirmed that electrode polarization dominated the impedance measurement. The enormous experimental permittivity $\left(1 / j \omega C_{0} Z_{\text {cell }}\right)$ reflected this. It would be experimentally very difficult to eliminate the electrode polarization contribution in a sea water impedance measurement.

In a sea ice medium, some pockets of brine exist in contact with the cell electrodes. These brine/electrode interfaces become polarized and contribute towards an electrode impedance. This contribution must be particularly large at high sea ice temperatures when the brine volume fraction is large. Experimental work summarised by Petrenko and Whitworth (1999) demonstrated that electrode polarization also occurs at an ice/electrode interface. However, the calculations performed in section 2.5 suggest that this process is minor in comparison to brine/electrode polarization and we assume it is negligible.

The dominance of electrode polarization in a sea ice impedance measurement depends on the fraction of the electrodes in contact with brine and the true electrical properties of the sea ice. The real component of the true permittivity of a sea ice sample is largely due to space charge polarization at internal ice/brine interfaces. This increases the right hand side of equation 3.6 and makes a sea ice impedance measurement much less susceptible to electrode polarization than a sea water measurement.

At this point a return to the literature is illuminating. Addison (1969) 
and Notz (2005) both commented on the contribution of electrode polarization in their low frequency sea ice ice impedance measurements. Notz assumed that it was overwhelmingly dominant at $2 \mathrm{kHz}$ between parallel wires. His wires were platinum, $120 \mathrm{~mm}$ long, $0.25 \mathrm{~mm}$ in diameter and separated by $5 \mathrm{~mm}$. The success of his salinity monitoring system gives weight to this assumption. The measurements of Addison (1969) were across gold plated, mesh, parallel plate capacitors which enclosed $7.6 \mathrm{~cm}$ diameter sea ice discs. Addison varied the spacing between the electrodes from $2-6 \mathrm{~cm}$, and showed that the measured permittivity was unchanged. This demonstrated that electrode polarization did not significantly contribute. The two systems have different geometries and sea ice microstructure scales differently in comparison. However, electrode polarization clearly contributes to a sea ice impedance measurement under some conditions.

The circumstances under which electrode polarization contributed significantly to a sea ice impedance measurement are unclear. The process warrants investigation. We sought to realise:

- the magnitude of the contribution,

- its frequency dependence, and

- its dependence on microstructure.

\subsubsection{High frequency impedance measurement error}

The Agilent 4294a impedance analyser employed offers excellent measurement accuracy. However, extension of the load beyond the instrument terminals degraded this accuracy. We went to considerable lengths in order to eliminate or minimise the measurement error. The preventative steps taken were:

- the utilization of coaxial measurement cables, 
- using the cables in the advised configuration (four-terminal pair),

- minimising measurement cable length, and

- characterising the cables by performing open/short/load reference impedance measurements to allow for open/short/load compensation.

These methods produced accurate impedance data up to approximately $200 \mathrm{kHz}$. At higher frequencies an impedance measurement error developed which degraded the accuracy of $Z^{\prime \prime}$ and caused it to become positive. This equates to a negative real permittivity which is entirely unphysical.

The failure of the open/short/load compensation technique to correct for this error identifies its origin - the error was due to reflections from the load and attenuation of the signal within the measurement cables [Agilent Technologies (2009)]. These processes can be accounted for by representing the measurement cables as a transmission line, of known length and propagation parameters. The model can be inverted to find the terminating impedance - which, in our case, would be the impedance of the measurement cell. The impedance analyser performs this for Agilent supplied test cables and fixtures but does not offer the capability for custom fabricated cables. However, the correction should be achievable in post processing.

\subsubsection{Gaining a physical insight}

Representing the electrical response of sea ice as a permittivity is not particularly illuminating. In our measurements, the real part of the permittivity was highly dispersive and spanned approximately seven decades across the measured frequency range. At low frequencies, the permittivities were many orders of magnitudes larger than those of brine and ice in isolation. This demonstrated that space charge polarization plays a dominating role in the electrical response of sea ice. The imaginary 
component $\epsilon^{\prime \prime}$ spanned approximately five decades and was featureless. This revealed that the conductive loss was much larger than the dielectric loss. The permittivity data showed no evidence of the dielectric relaxation which occurs in pure ice at $\sim 10 \mathrm{kHz}$.

The transformation from an impedance to a permittivity is affectively a division by $\omega$. This $1 / \omega$ dependence then masks many of the features which appeared prominently in the impedance spectrum. The reason the material response was represented in terms of a complex permittivity was that, it is clear what the real and imaginary components physically mean. The real component describes polarization and the imaginary component describes loss (both dielectric and conductive). We sought to find a more illuminating representation of the electrical response of sea ice.

\subsubsection{Realistic sea ice and non-invasive measurement cells}

The main limitation of the lab freezer experiment was the small size of the container in which the ice was grown. The container constrained the brine rejected during sea ice formation and produced unnaturally high salinity sea ice. A portion of this brine was squeezed to the top of the container where it sat in a pool. Although the container insulation was sufficient to regulate one-dimensional cooling, we observed none of the impedance anisotropy which occurs in natural, columnar sea ice. We sought to produce more realistic sea ice.

The solid plate capacitors were obviously obstructive to natural sea ice formation. They constrained a portion of sea ice and blocked brine drainage. Mesh electrodes are considered a less invasive sensor. 


\section{Chapter 4}

\section{Wheelie bin experiment}

This chapter documents the experimental and analytical culmination of this MSc project. The wheelie bin experiment was built on the back of the lab freezer experiment - its design incorporated many of the lessons learned previously and it aimed to investigate the questions outlined in section 3.3. It involved less invasive temperature and impedance monitoring of a more realistic sea ice sample. The experiment revealed the true low frequency electrical properties of sea ice. These properties were formulated in terms of a broadband physical model which offers new insight into the electrical response of sea ice. This understanding was linked with a microstructural model.

\subsection{Experimental description}

\subsubsection{Growing realistic sea ice}

Natural sea ice grows one-dimensionally from the top of the ocean by heat loss to the atmosphere. The vast majority of high salinity brine produced during ice formation is dissipated into the underlying ocean and only a small fraction becomes incorporated in the sea ice itself. The challenge of 'growing' realistic laboratory sea ice is to replicate these processes in an 
enclosed container in a freezer.

One dimensional ice growth can be forced by insulating the container sides and bottom to block heat flux through these surfaces. The ice remains exposed to cold air at the top of the container. The thermal conductance of the insulation must be sufficiently small so that, even when a thick layer of ice separates the sea water from the cold air, vertical heat transfer through the ice remains the dominant heat loss process.

Maintaining the salinity of the unfrozen sea water approximately constant is a more difficult task. One solution is to have a tall container which gives a large volume of water in which the brine can be dissipated. If the ice studied is restricted to the top of the container, which forms before the salinity of the water has increased drastically, then the brine volume fractions should be realistic. A better apparatus would remove water from a salty base layer and replace it with standard sea water of the same temperature, effectively simulating the role of an underlying ocean. Such a system would require insulated hoses, a drain, and a pump and was deemed too complex for this experiment.

The sea ice produced was grown in a 360L wheelie bin with dimensions $1100 \mathrm{~mm}(\mathrm{H}) \times 620 \mathrm{~mm}(\mathrm{~W}) \times 850 \mathrm{~mm}(\mathrm{D})$. It is pictured in Figure 4.1 . The profile of the bin is narrow and tall and it encloses a much larger volume than the container used in the lab freezer experiment. It should produce much more realistic sea ice. The bin is free standing, and is made of strong HDPE plastic. The sides were lined with $\sim 5 \mathrm{~cm}$ thick polystyrene slabs and the voids filled with expanding foam. The thermal conductivity of sea ice is $\sim 2 \mathrm{~W} / \mathrm{m} \mathrm{K}$ [Pringle (2004)] and polystyrene is $0.033 \mathrm{~W} / \mathrm{m} \mathrm{K}$ [Lide (1993)]. Therefore, the thermal conductance of the $5 \mathrm{~cm}$ insulative layer is the equivalent of $3 \mathrm{~m}$ of sea ice. The wheels were removed from the bin and the plastic bottom sat flush on a concrete slab which provided the ground insulation. The wheelie bin was positioned in a laboratory cold room at Industrial Research Limited in Gracefield, Lower Hutt. The room had a floor area of approximately $10 \mathrm{~m}^{2}$ and offered precise temperature control. 
The wheelie bin was positioned in a corner of the room and filled with approximately $200 \mathrm{~L}$ of sea water. The sea water was sourced at the local Days Bay which is on the eastern side of Wellington harbour.

\subsubsection{Temperature monitoring}

If the side insulation is sufficient then the thermal profile is one-dimensional with temperature varying only vertically. A vertical thermistor string was installed within the wheelie bin to monitor this profile. The thermistor string follows the design of Pringle (2004) and included eight thermistors spaced at regular intervals along its $1 \mathrm{~m}$ length. The thermistors, and their constantan measurement wires, are tied to a teflon spine which slots inside a thin walled stainless steel tube filled with vegetable oil. The thermal conductance of the structure matches that of the surrounding sea ice, and so, the temperature measurements should be non-invasive. Such care was not taken in the lab freezer experiment. The thermistor string was held vertically just off the centre of the container by a ply wood shelf which sat inside the wheelie bin (pictured in Figure 4.1(b)). A National Instruments USB6009 DAQ board was used to log the thermistors temperatures. The device measured the potential drop across the thermistors which formed a potential divider with a known resistance. This network was maintained at a constant potential which allowed the thermistor resistance to be calculated. The temperature was inverted from the known resistance using the expression of Pringle (2004).

The wheelie bin was not filled to full capacity and the water level was approximately $70 \mathrm{~cm}$ above the bottom of the container. The thermistor spacing was not optimal for this fill level. This coupled with a thermistor connection failure meant that only four thermistors were situated within the ice. 

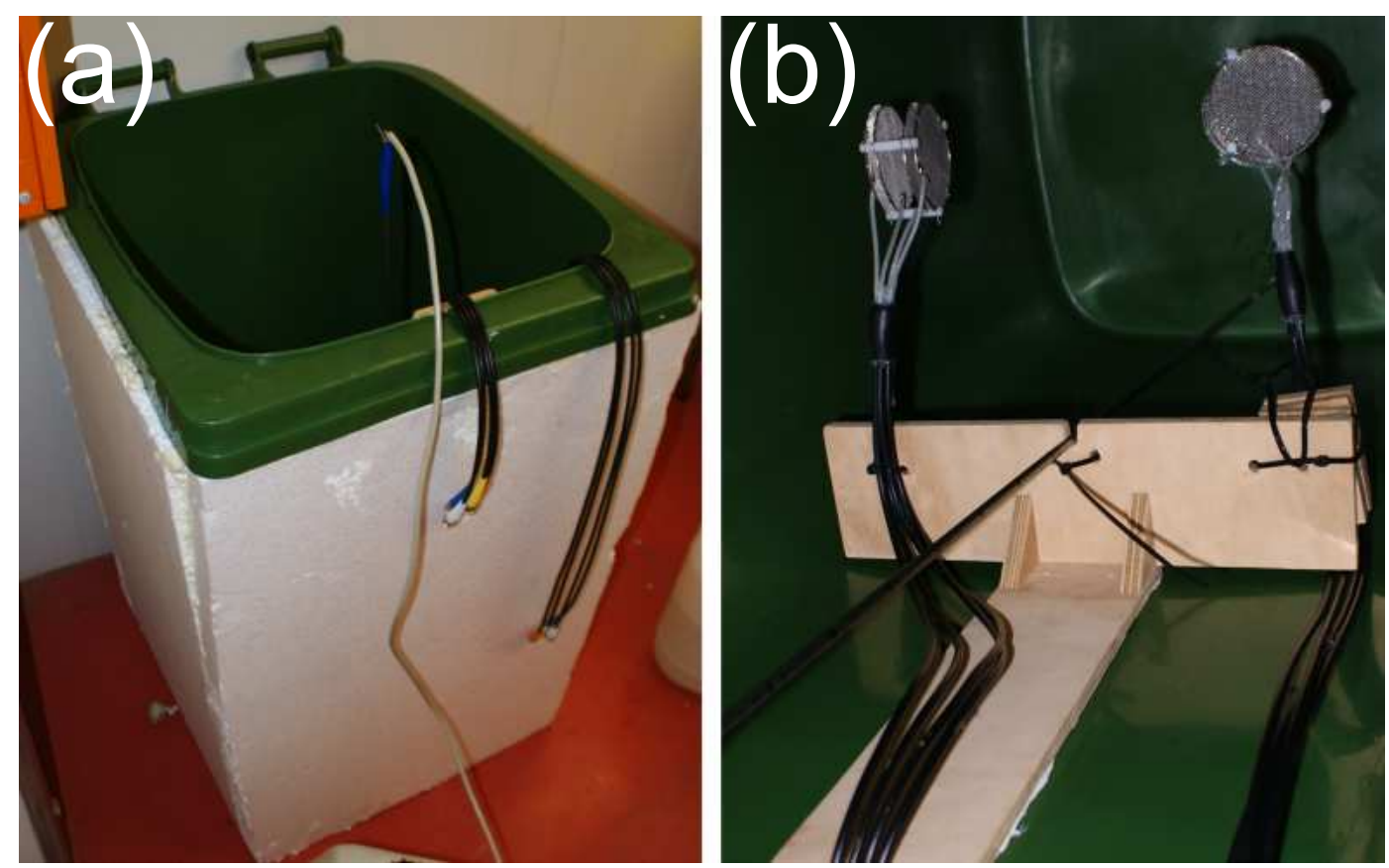

Figure 4.1: (a) shows the insulated wheelie bin inside the cold room with measurement cables extending out. (b) shows the placement of capacitors and the thermistor string.

\subsubsection{Impedance measurements using the four-electrode capacitive cell}

Sea ice impedance measurements were performed using a four-electrode capacitive cell (4ECC) and an Agilent 4294A impedance analyser. The cell (pictured in Figure 4.2) is a custom design, inspired by the description in Chelidze et al. (1999). It was constructed by the Author and technical staff at VUW. The device a significant advancement on the solid parallel plate capacitors used in the lab freezer and Antarctic experiments as it offers the capability to realise and eliminate the parasitic electrode impedance. The cell is comprised of four parallel mesh discs bound together by three nylon rods and stainless steel wire. A measurement cable connects directly to each electrode. The mesh material minimises the cell's invasiveness. 


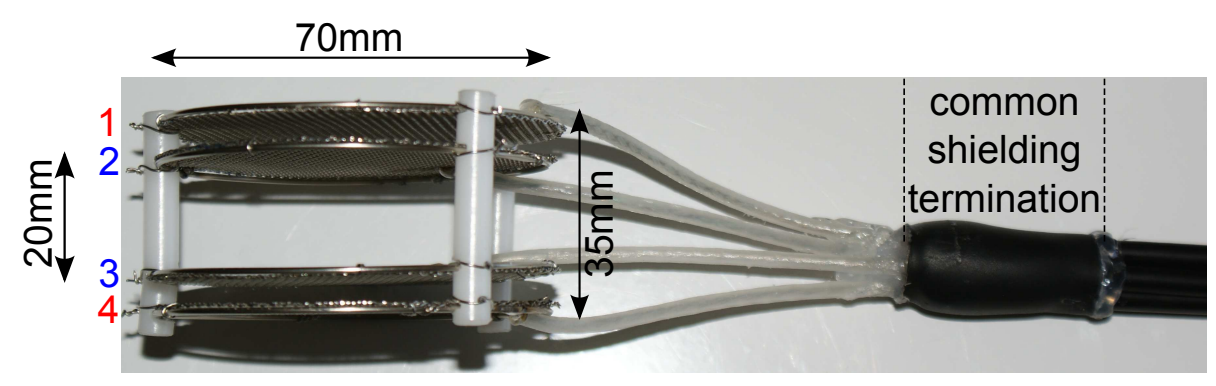

Figure 4.2: The four-electrode capacitive cell (4ECC).

The cell can be used in two modes of operation. It can perform:

- a four-electrode measurement, or

- a two-electrode measurements across any combination of electrodes.

A four-electrode measurement is made by injecting current into the outer electrodes $(1 \& 4)$ and measuring the potential difference across the inner two $(2 \& 3)$. This measurement is not sensitive to electrode impedance as only the outer electrodes become polarized, while the inner electrodes measure the true potential across the medium. The measurement can be represented by the following circuit:

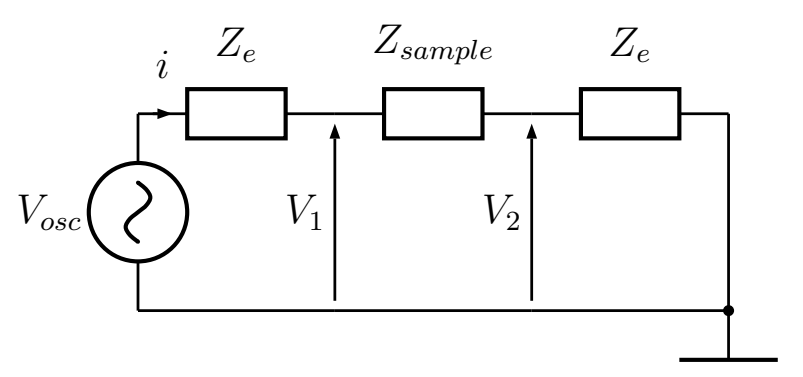

Figure 4.3: Equivalent circuit representation of a four-electrode measurement.

The electrode impedance is represented by $Z_{e}$ and the true impedance of the sample by $Z_{\text {sample }}$. The measured impedance is then:

$$
\frac{V_{1}-V_{2}}{i}=Z_{\text {sample }} \text {. }
$$


A two electrode measurement is sensitive to the parasitic elements $Z_{e}$ and yields:

$$
\frac{V_{\text {osc }}}{i}=Z_{e}+Z_{\text {sample }}+Z_{e}
$$

In this experiment both four-electrode and two-electrode measurements were made so that the contribution of $Z_{e}$ could be investigated.

The measurement cables that connected with the cell were coaxial and the internal wire was solder connected directly to each mesh electrode. The internal wire insulation extended to within $0.5 \mathrm{~cm}$ of each electrode and the coaxial shielding terminated communally for the four wires approximately $7 \mathrm{~cm}$ away from the electrodes. The cable shielding termination was electrically insulated with RTV silicon, hot glue and heat shrink. Male BNC connectors were fitted at the instrument end of the cables. How these connected with the instrument controlled what type of measurement was performed. Figure 4.4 demonstrates the cable configuration for a four-electrode and a two-electrode measurement. The measurement cables connect for the two-electrode measurement in a twoterminal configuration.

A high speed differential amplifier (shown in Figure 4.4a) was included to increase the input impedance of the potential-sensing connections for the four-electrode measurement. Without this device the potential connections drew and sank considerable current at high frequencies. The amplifier has a gain of unity and follows the design example in Analog Devices (2009). A short $(27 \mathrm{~cm})$ coaxial cable extended from the high potential terminal to a BNC T-junction connector at the high current connection. This connection was replicated on the low current and voltage terminals in order to perform a two-electrode measurement as shown in Figure 4.4(b).

The cells were calibrated to enable the measured impedance to be related to the material properties (e.g. permittivity). A general relationship 


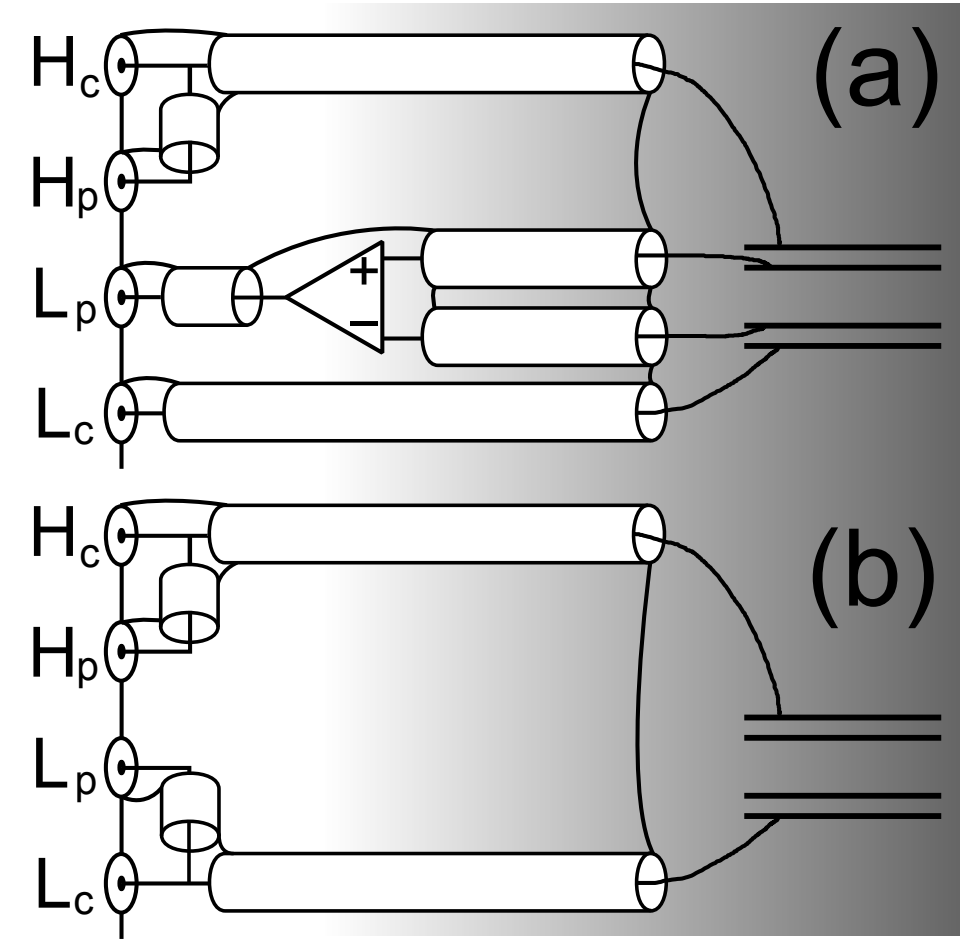

Figure 4.4: (a) shows the cable configuration for a four-electrode measurement. (b) shows the cable configuration for a two-electrode measurement (in this case the impedance is measured between electrodes $1 \& 4)$. The distance scale is meaningless and all cables extending to the cell were the same length.

between impedance and material property for a particular capacitor is:

$$
Z=\frac{1}{j \omega G \epsilon_{0} \epsilon^{*}}
$$

where $G$ is some geometric factor (with units [m]) which characterises the electric field in the space between and around the plates. In the lab freezer experiment $G$ was calculated, and the cells calibrated, by measuring the impedance of a load with a known permittivity - the air. For the fourelectrode capacitive cells, a more accurate value of $G$ was calculated from impedance measurements of four standard loads. These loads were: 
- de-ionized water at $20^{\circ} \mathrm{C}\left(\epsilon^{\prime}=80.2 \times \epsilon_{0}\right.$ - measurement made at high frequency to negate electrode polarization),

- Air $\left(\epsilon=\epsilon_{0}\right.$ - this measurement was not possible for the four-electrode measurement configuration),

- sea water at $17^{\circ} \mathrm{C}\left(\sigma^{\prime}=4.5\left[\mathrm{Sm}^{-1}\right]\right.$ - assuming standard salinity and using the expressions of Janz and Singer (1975)), and

- sea water at $0^{\circ} \mathrm{C}\left(\sigma^{\prime}=2.0\left[\mathrm{Sm}^{-1}\right]\right.$ - assuming standard salinity and using the expressions of Janz and Singer (1975)).

Table 4.1 summarises the cell geometrical factors, averaged for the above measurements.

\begin{tabular}{|c|c|c|c|}
\hline electrode combination & plate separation $[\mathrm{mm}]$ & $\mathrm{G}[\mathrm{m}]$ & $\frac{A}{d}[\mathrm{~m}]$ \\
\hline $1-2$ & 7 & 0.76 & 0.55 \\
\hline $2-3$ & 20 & 0.30 & 0.20 \\
\hline $1-4$ & 35 & 0.24 & 0.11 \\
\hline $4 \mathrm{E}$ (four-electrode) & 20 & 0.45 & 0.2 \\
\hline
\end{tabular}

Table 4.1: Cell calibration factors.

Two identical measurement cells were constructed and arranged in horizontal and vertical orientations within the container. They can be seen in Figure 4.1(b) suspended from the wooden shelf and extending into the centre of the wheelie bin. They were positioned so that their centres were $12.5 \mathrm{~cm}$ below the fill level. The measurement cables connecting to the vertically orientated capacitor were $92 \mathrm{~cm}$ long, and $133 \mathrm{~cm}$ long connecting to the horizontal capacitor.

The impedance measurement sequence for each cell throughout the experiment was:

- two-electrode measurements across electrode pairs 1-2, 2-3 \& 1-4, and 
- four-electrode measurement across the entire cell.

The impedance analyser settings were:

\begin{tabular}{|l|r|}
\hline Start frequency & $40 \mathrm{~Hz}$ \\
\hline Stop frequency & $10 \mathrm{MHz}$ \\
\hline Frequency scale & logarithmic \\
\hline Measurement points & 201 \\
\hline Bandwidth & 5 \\
\hline $\mathrm{V}_{\text {osc }}$ & $500 \mathrm{mV}$ \\
\hline
\end{tabular}

Note that the Bandwidth setting is a device particular setting which maximises the measurement accuracy. It is not $5 \mathrm{~Hz}$. The settings configure an impedance measurement sweep at 201 logarithmically spaced frequencies between $40 \mathrm{~Hz}$ and $110 \mathrm{MHz}$. The applied voltage is $500 \mathrm{mV}_{R M S}$.

A set of measurements took on average 20 minutes to complete and required moving the impedance analyser, high speed amplifier, and power supply (for the amplifier) into the cold room. The equipment was arranged on a trolley which could be wheeled through the door of the cold room. A polystyrene box was constructed to insulate the impedance analyser and there was no evidence of the instrument accuracy being degraded by the extreme operating temperatures. Device calibration was performed with an Agilent $50 \Omega$ reference load before the wheelie bin experiment began and its accuracy was confirmed some time later once the instrument was chilled.

The controlled air temperature sequence which forced the ice growth was:

- $0 \rightarrow 50$ hours $=0^{\circ} \mathrm{C}$

- $50 \rightarrow 330$ hours $=-20^{\circ} \mathrm{C}$

- $330 \rightarrow 460$ hours $=-25^{\circ} \mathrm{C}$. 


\begin{tabular}{|c|c|c|}
\hline Measurement number & Time elapsed [hrs] & Calculated cell temperature $\left[{ }^{\circ} \mathrm{C}\right]$ \\
\hline 1 & 0 & 17.4 \\
\hline 2 & 67 & -0.3 \\
\hline 3 & 91 & -1.9 \\
\hline 4 & 114.5 & -2.1 \\
\hline 5 & 144 & -2.3 \\
\hline 6 & 163.5 & -3.0 \\
\hline 7 & 239.5 & -8.1 \\
\hline 8 & 264 & -9.5 \\
\hline 9 & 288 & -10.9 \\
\hline 10 & 310.5 & -12.3 \\
\hline 11 & 331 & -13.6 \\
\hline 12 & 407 & -21.3 \\
\hline 13 & 432.5 & -22.4 \\
\hline 14 & 456 & -23.0 \\
\hline
\end{tabular}

Table 4.2: Impedance measurement occasions and estimated cell temperatures.

Impedance measurements were conducted on 14 occasions over a three week period. Ice formation had begun by the time of the third measurement. The measurement times and estimated cell temperatures (defined in section 4.2.1) are summarised in Table 4.2. 


\subsection{Results and Analysis}

\subsubsection{Temperature profile and observed sea ice microstructure}

Figure 4.5 shows the recorded temperature series of the four thermistors situated within the ice. The insert shows a vertical cross section of the position of the thermistors in relation to the impedance measurement cells - the $\mathrm{z}$ axis corresponds to the distance below the initial water level. The encapsulation of a thermistor in ice (freeze-in) is indicated by a sudden change in the temperature gradient.

The data series show that the water cooled uniformly to $-1.8^{\circ} \mathrm{C}$ before ice began to form. A surface layer of ice was observed from the time of the third impedance measurement. The bottom thermistor froze-in before $100 \mathrm{hrs}$ indicating that the ground insulation was insufficient. Ice formation caused a salinity increase in the remaining water, suppressing its freezing point and the remaining liquid cooled below $-1.8^{\circ} \mathrm{C}$. The top thermistor froze-in at 150 hours and the subsequent thermistor at an estimated time of $210 \mathrm{hrs}$ (the exact freeze time is unknown due to a failure in the data logging equipment). The third thermistor froze-in some time later. In the top $40 \mathrm{~cm}$ of the container a one dimensional temperature profile was observed which was interpolated to produce a 'calculated temperature series' $\left(\mathrm{T}_{C}\right)$ at position $\mathrm{z}_{c}$, the vertical centre of the cells. This profile demonstrates that at approximately 160 hours the cells became enclosed in ice and the vertical temperature variation across the cell was not more, and generally much less, than $\sim 2^{\circ} \mathrm{C}$. The calculated temperature data is summarised in Table 4.2.

Sea ice formed at these suppressed temperatures from higher salinity water will likely have a higher brine volume fraction than natural ice although this ultimately depends on the efficiency of brine drainage into the underlying water. 


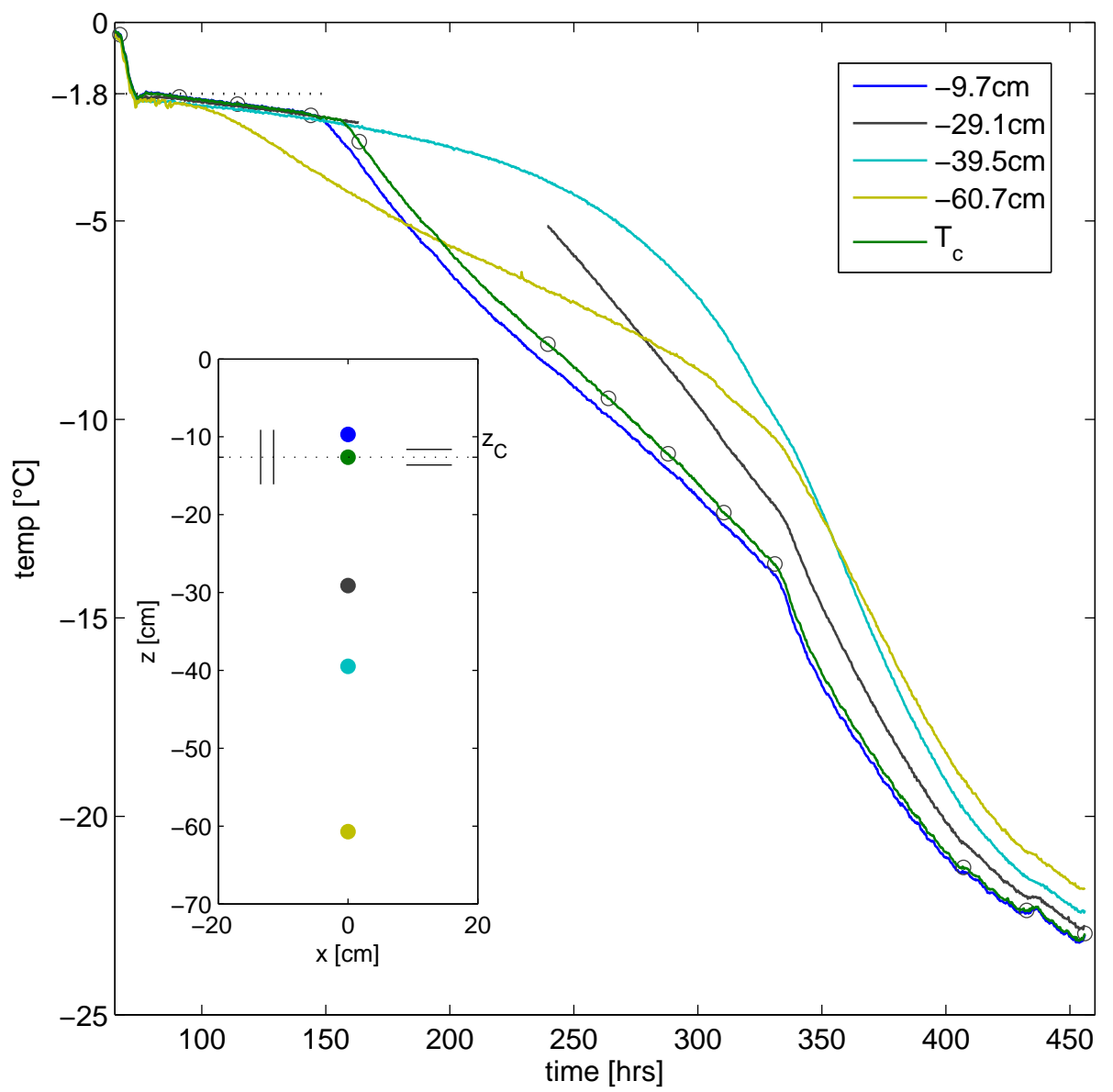

Figure 4.5: The vertical temperature profile within the wheelie bin. The open circles represent calculated cell temperatures at impedance measurement times as summarised in Table 4.2. [The insert shows a vertical cross section of the arrangement of thermistors (the colour of the solid circles' corresponds to the temperature series at that depth) and capacitors within the wheelie bin. The $x$ axis corresponds to displacement from the vertical centre of the container and the $\mathrm{z}$ axis to distance below the water level in $\mathrm{cm}$ at the start of the experiment.] 


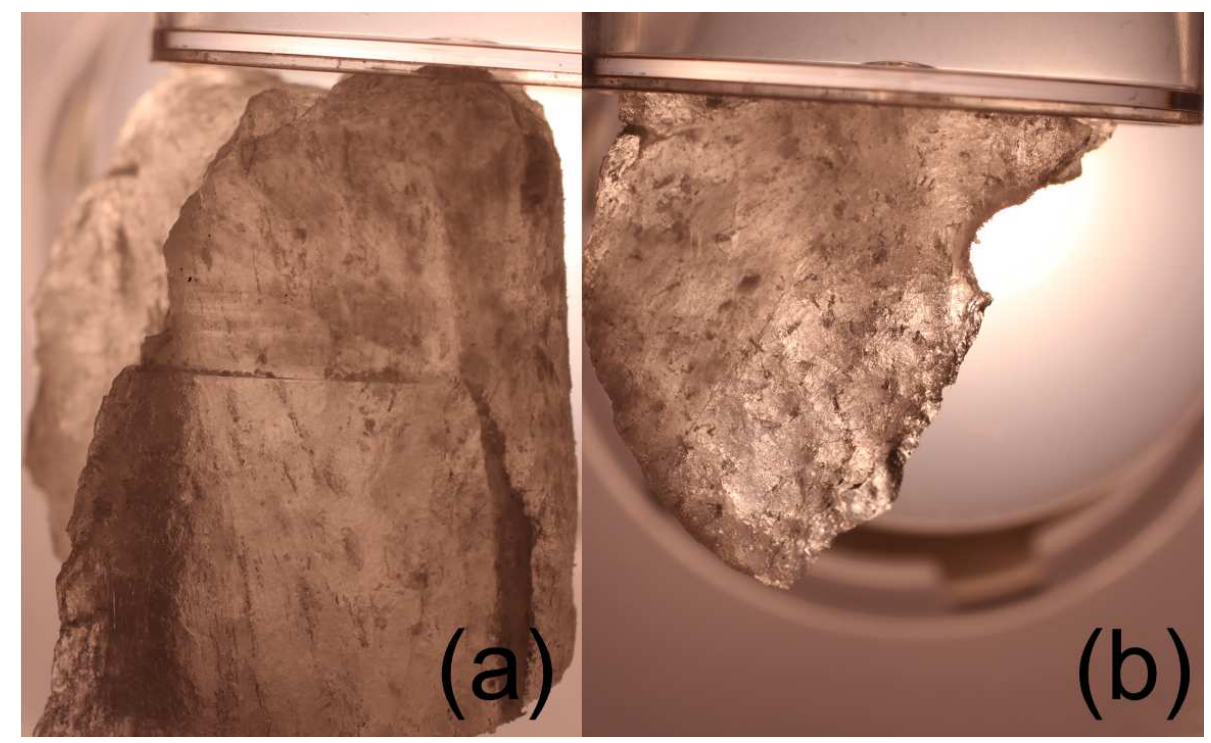

Figure 4.6: A sea ice core taken at the conclusion of the experiment revealed vertically elongated brine pockets. (a) shows a vertical cross section and (b) a horizontal cross section.

For most of the experiment a small brine pool sat on top of the ice. This liquid froze when the air temperature was dropped to $-25^{\circ} \mathrm{C}$. At the conclusion of the experiment an ice core was taken at the depth of the measurement cells. Figure 4.6 shows the vertically aligned and elongated brine pockets observed. 


\subsubsection{Sea ice impedance data}

Figure 4.7 and 4.8 display compensated impedance data for the 1-2 twoelectrode measurements and the four-electrode measurements at selected cell temperatures. The two-electrode data across electrode pairs 2-3 and 1-4 are included in Appendix B. The data are presented as horizontal and vertical components of the impedance, as measured by the vertical and horizontal capacitors. This convention is used exclusively from this point on.

Open/short/load compensation was performed on the two-electrode data and open/short compensation on the four-electrode data (the load measurement is ill-defined in the case of a four-electrode measurement). A high frequency impedance measurement error which affects $Z^{\prime \prime}$ limits the usable frequency range. The magnitude of this error depends on the size of the impedance load and is particularly apparent in low impedance measurements. A transition of $Z^{\prime \prime}$ from negative to positive indicates the upper frequency limit of the measured impedance.

The real measured impedance is accurate up to $1 \mathrm{MHz}$ - it was less sensitive to the measurement error. The imaginary component upper frequency limit for the different measurement configuration's is as follows:

\begin{tabular}{|c|c|}
\hline two-electrode $1-2$ & $1 \mathrm{MHz}$ \\
\hline two-electrode $2-3,1-4$ & $100 \mathrm{kHz}$ \\
\hline four-electrode & $100 \mathrm{kHz}$ \\
\hline
\end{tabular}



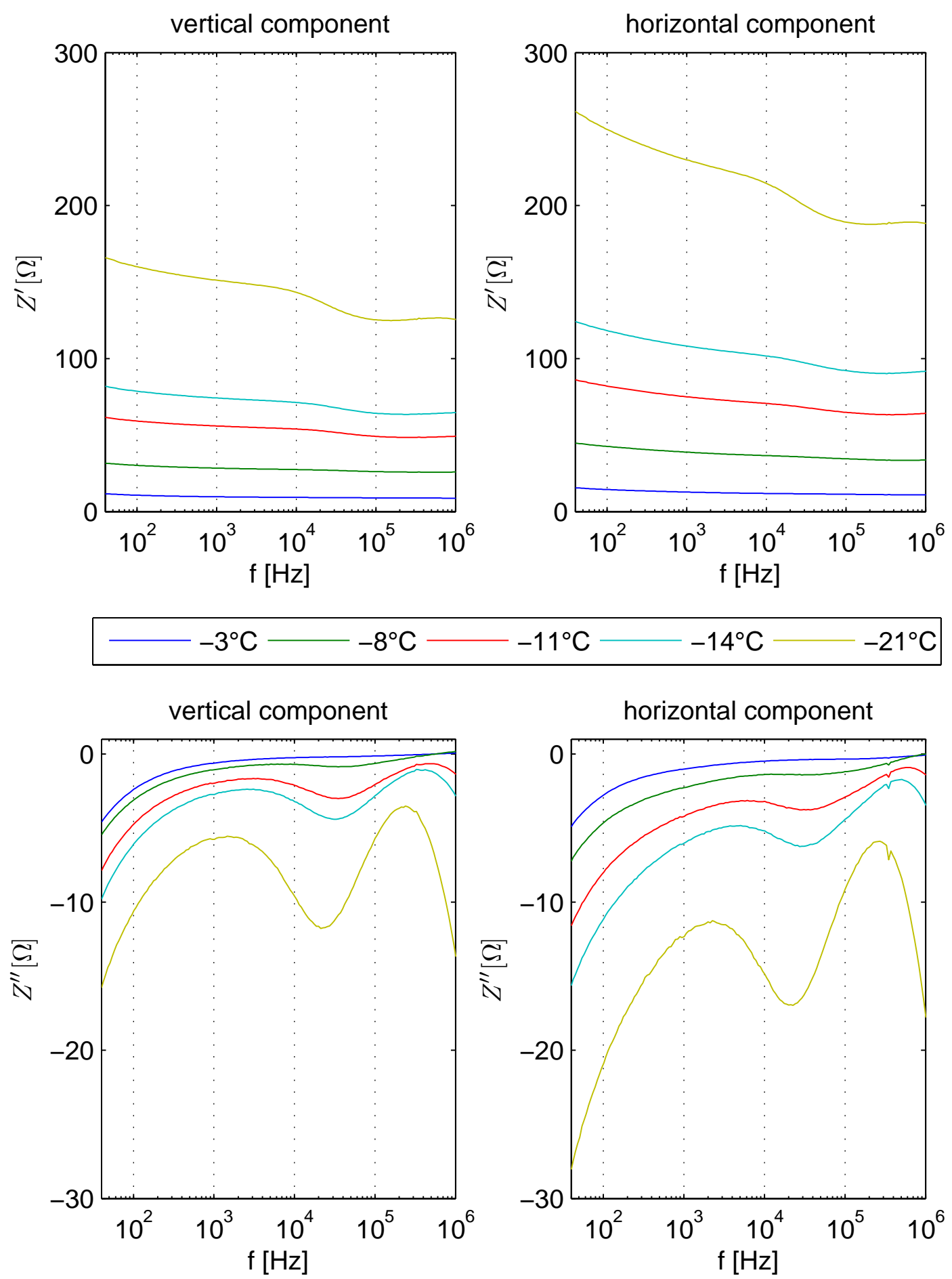

$-11^{\circ} \mathrm{C}-14^{\circ} \mathrm{C}-21^{\circ} \mathrm{C}$

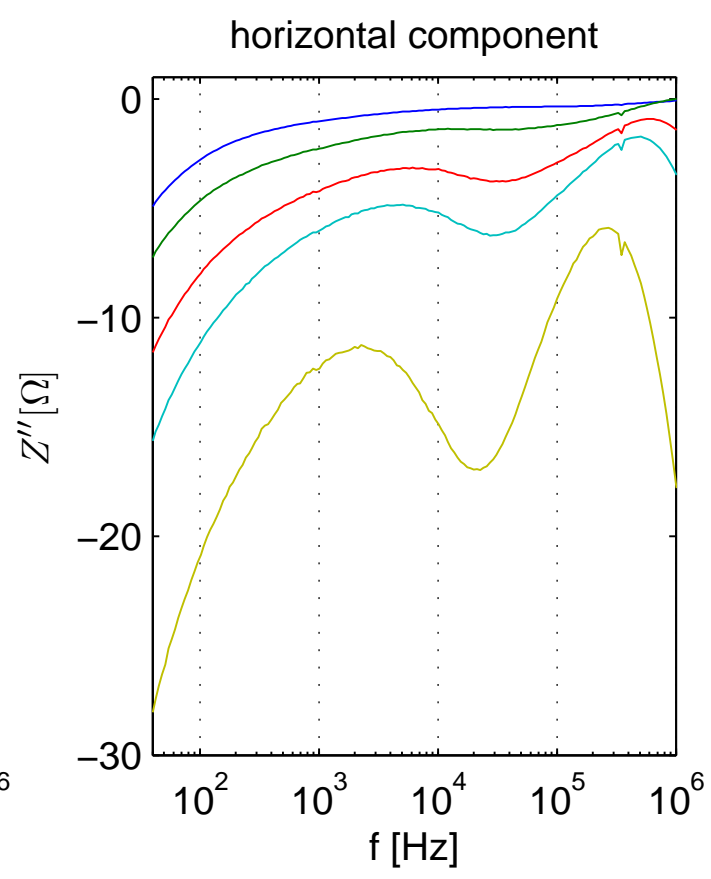

Figure 4.7: Vertical and horizontal components of sea ice impedance for a two-electrode measurement across electrode pair 1-2. 

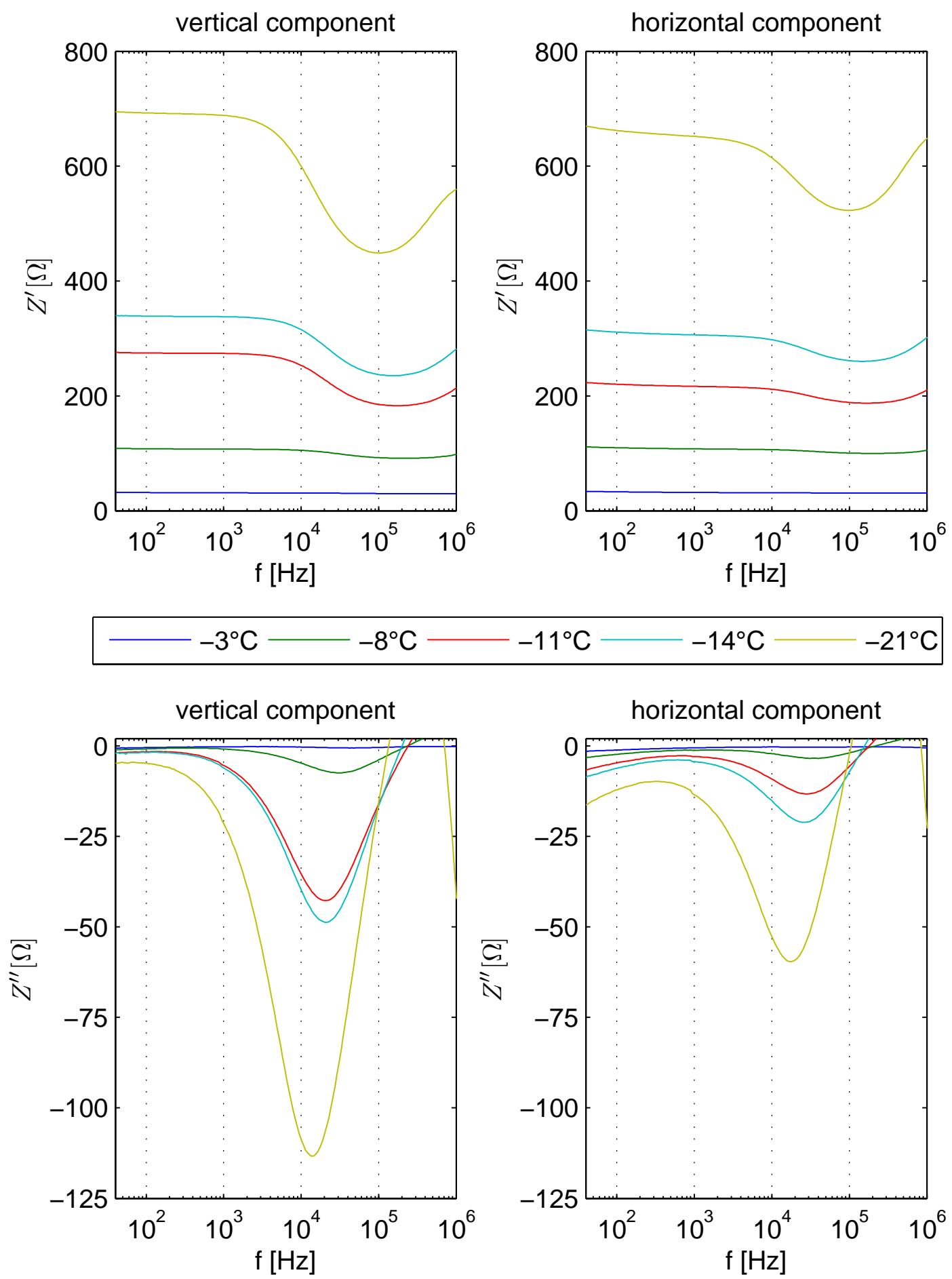

$-11^{\circ} \mathrm{C}--14^{\circ} \mathrm{C}--21^{\circ} \mathrm{C}$

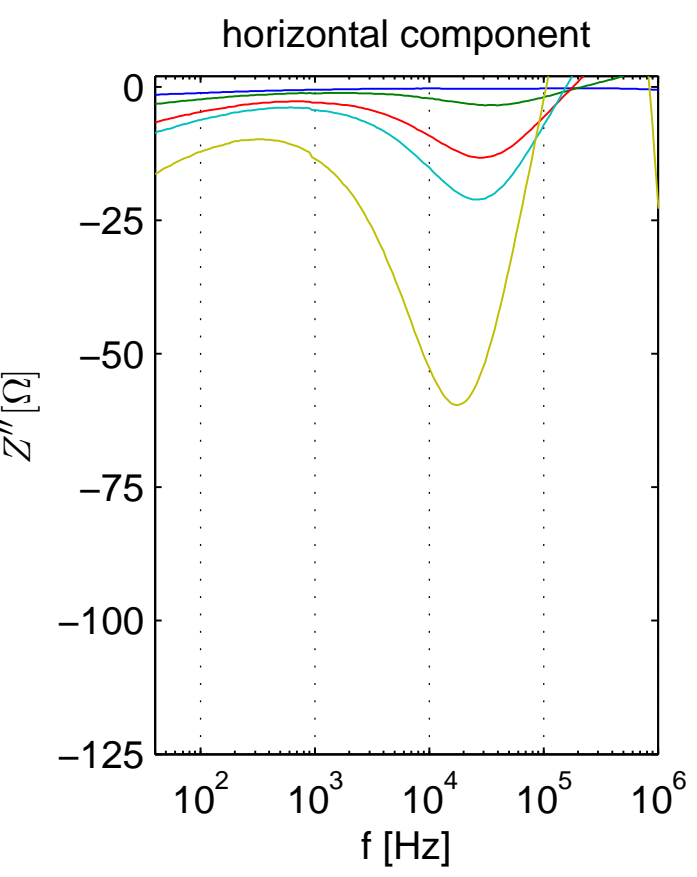

Figure 4.8: Vertical and horizontal components of sea ice impedance for a four-electrode measurement. 


\subsubsection{Electrode polarization and the performance of the four-electrode capacitive cell}

The four-electrode capacitive cell was designed to investigate the affect of electrode polarization on a sea ice impedance measurement. If the four electrode measurement works as desired then it should measure the true impedance of the sea ice sample. Impedance measurements performed at varied electrode spacings provide an alternative way to decouple the the parasitic electrode impedance from the sample impedance. Throughout the experiment, two-electrode impedance measurements were made across the electrode pairs 1-2, 2-3 and 1-4, each of which enclose a different volume of sea ice and thus measure a different impedance. Assuming the electrode impedance $Z_{e}$ is the same for all the measurements and the medium is homogeneous then the measured impedances can be represented as:

$$
\begin{aligned}
& Z_{1-2}=Z_{e}+\frac{1}{j \omega \epsilon_{0} G_{1-2} \epsilon^{*}} \\
& Z_{2-3}=Z_{e}+\frac{1}{j \omega \epsilon_{0} G_{2-3} \epsilon^{*}} \\
& Z_{1-4}=Z_{e}+\frac{1}{j \omega \epsilon_{0} G_{1-4} \epsilon^{*}} .
\end{aligned}
$$

The subscript parameters 1-2, 2-3 and 1-4 denote the different electrode combinations. The electrical response of the medium is represented in terms of a complex permittivity and $\mathrm{G}$ is the geometric factor as given in Table 4.1. Rearranging equations 4.4 shows that the permittivity can be recovered from any two two-electrode combinations.

$$
\begin{aligned}
& j \omega \epsilon_{0} \epsilon^{*}=\frac{\frac{1}{G_{1-4}}-\frac{1}{G_{2-3}}}{Z_{1-4}-Z_{2-3}} \\
&=\frac{1}{G_{2-3}}-\frac{1}{G_{1-2}} \\
& Z_{2-3}-Z_{1-2} \\
&=\frac{1}{G_{1-4}}-\frac{1}{G_{1-2}} \\
& Z_{1-4}-Z_{1-2}
\end{aligned}
$$


Figure 4.9 compares the material properties obtained using the variable spacing method (with electrode combinations 1-2 and 1-4 - the third line in equation 4.5) with the four-electrode method. The data are of the horizontal component of $-8^{\circ} \mathrm{C}$ sea ice. The plot compares these material properties with single two-electrode measurements for electrode combination 1-2 and 1-4.
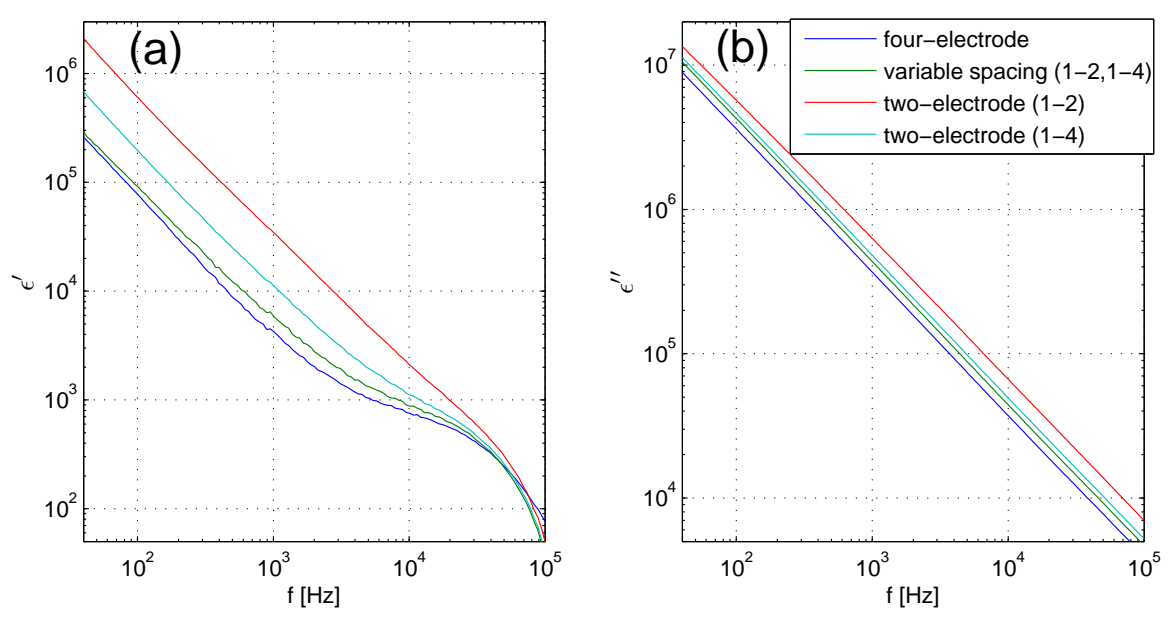

Figure 4.9: Comparison of the material properties obtained from a fourelectrode measurement, the variable spacing procedure and raw twoelectrode measurements.

Two factors suggest that the four-electrode cell measured the true sample impedance and not the electrode impedance. These are:

- the four-electrode and variable spacing methods return similar results, and

- the four-electrode permittivity $\epsilon^{\prime}$ is considerably less than the twoelectrode measurements. The measured polarization is certainly reduced.

This result was typical of all sea ice impedance measurements. 
The performance of the four-electrode cell was further investigated with an impedance measurement of $18.2 \mathrm{M} \Omega$ de-ionized water. If the measurement is insensitive to electrode polarization then the dielectric constant should be $\sim 100$ and independent of frequency. Figure (4.10) shows that at low frequencies this was not the case. Both the variable

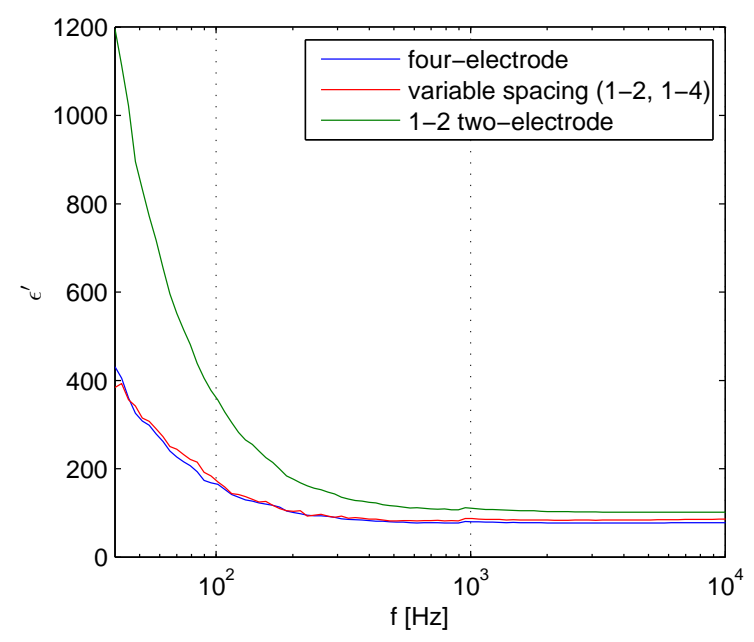

Figure 4.10: Dielectric constant of de-ionized water as measured with the four-electrode capacitive cell.

spacing method and the four-electrode method failed at low frequencies to measure the true permittivity of the medium. Their low frequency permittivity was slightly less than that of a two-electrode measurement suggesting that the measured electrode impedance had been reduced. This demonstrates the limitations of the cell - when deployed in even the lowest ionically concentrated liquid it fails to completely eliminate the electrode impedance. In high salinity sea water, electrode polarization still dominates the electrical response across the entire measurement frequency range. However, in a sea ice medium the four-electrode measurement appears to reduce electrode polarization to an extent where it is overwhelmed by material polarization and the device functions as required - the condition of equation 3.6, is not satisfied. 


\subsubsection{Sea ice permittivity data}

The permittivity data are presented in Figures 4.11 and 4.12 for both the two-electrode measurement across the electrode pair 1-2 and the fourelectrode measurements across both capacitors (the data for the other twoelectrode configuration's are included in Appendix B). These data sets provide contrasting examples of the contribution of electrode polarization - it is absent for the four-electrode measurement and dominant at low frequencies for the two-electrode measurement. Also, the impedance measurement error is less prominent in the two-electrode data and it retains accuracy at higher frequencies $(<1 \mathrm{MHz})$ - a limit where the parasitic electrode combination is absent.

The permittivity is strongly dispersive. At low frequencies, the real component of the four-electrode permittivity is considerably less than that of the two-electrode illustrating the dominance of electrode polarization. With this dominance removed, the four electrode data shows $\epsilon^{\prime}$ flattens off at mid frequencies, a feature which becomes more prominent at lower temperatures. The high frequency $\epsilon^{\prime}$ data should flatten off below 100 [Addison (1969)]. This is not the case for high temperature two-electrode measurements and all the four-electrode measurements. These still suffer from the impedance measurement error. For both measurement types, $\epsilon^{\prime \prime}$ exhibits $1 / \omega$ dependence across the entire measurement range. It shows some evidence of dielectric loss at high frequencies for the four-electrode data. 

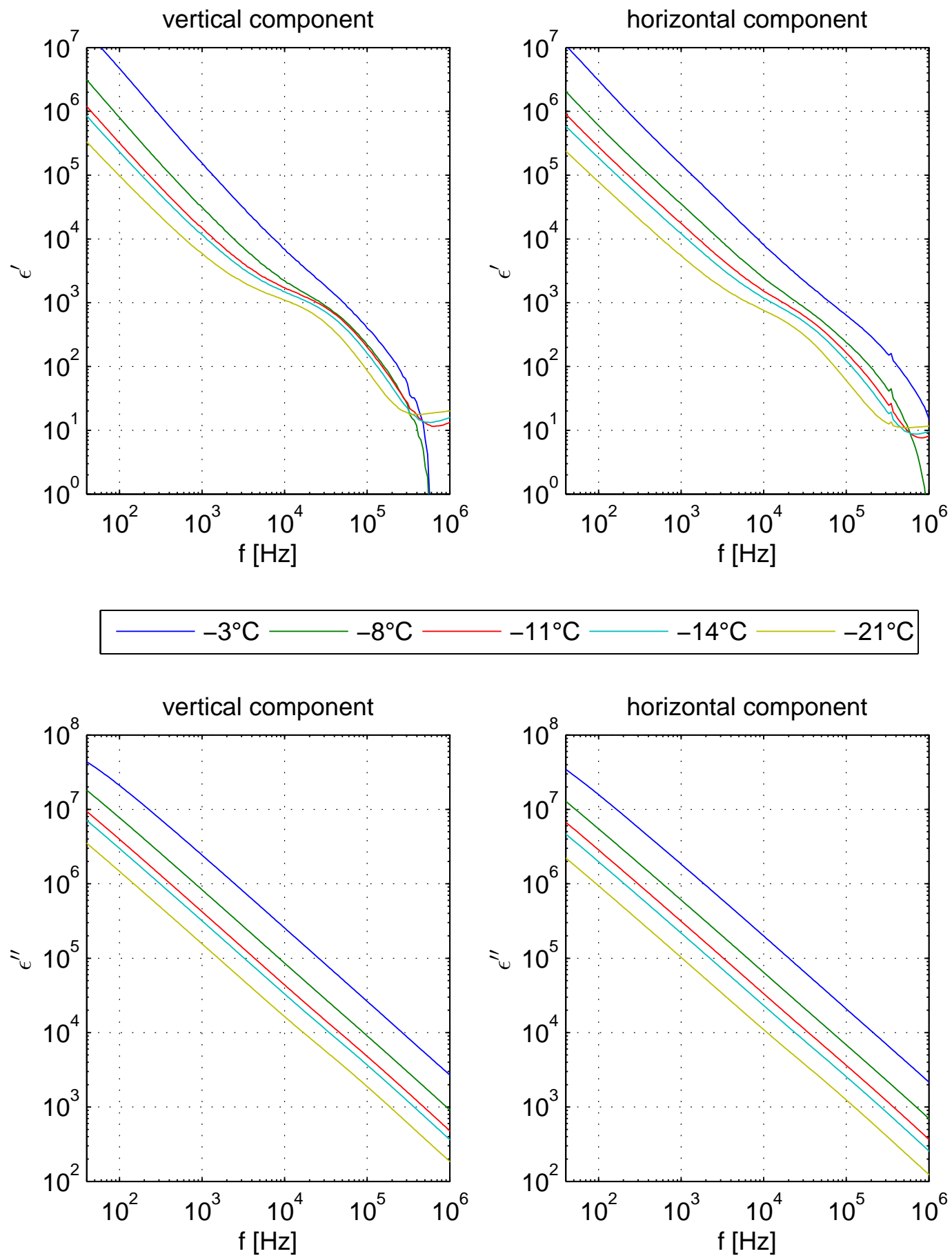

Figure 4.11: Vertical and horizontal components of sea ice permittivity for a two-electrode measurement across electrode pair 1-2. 

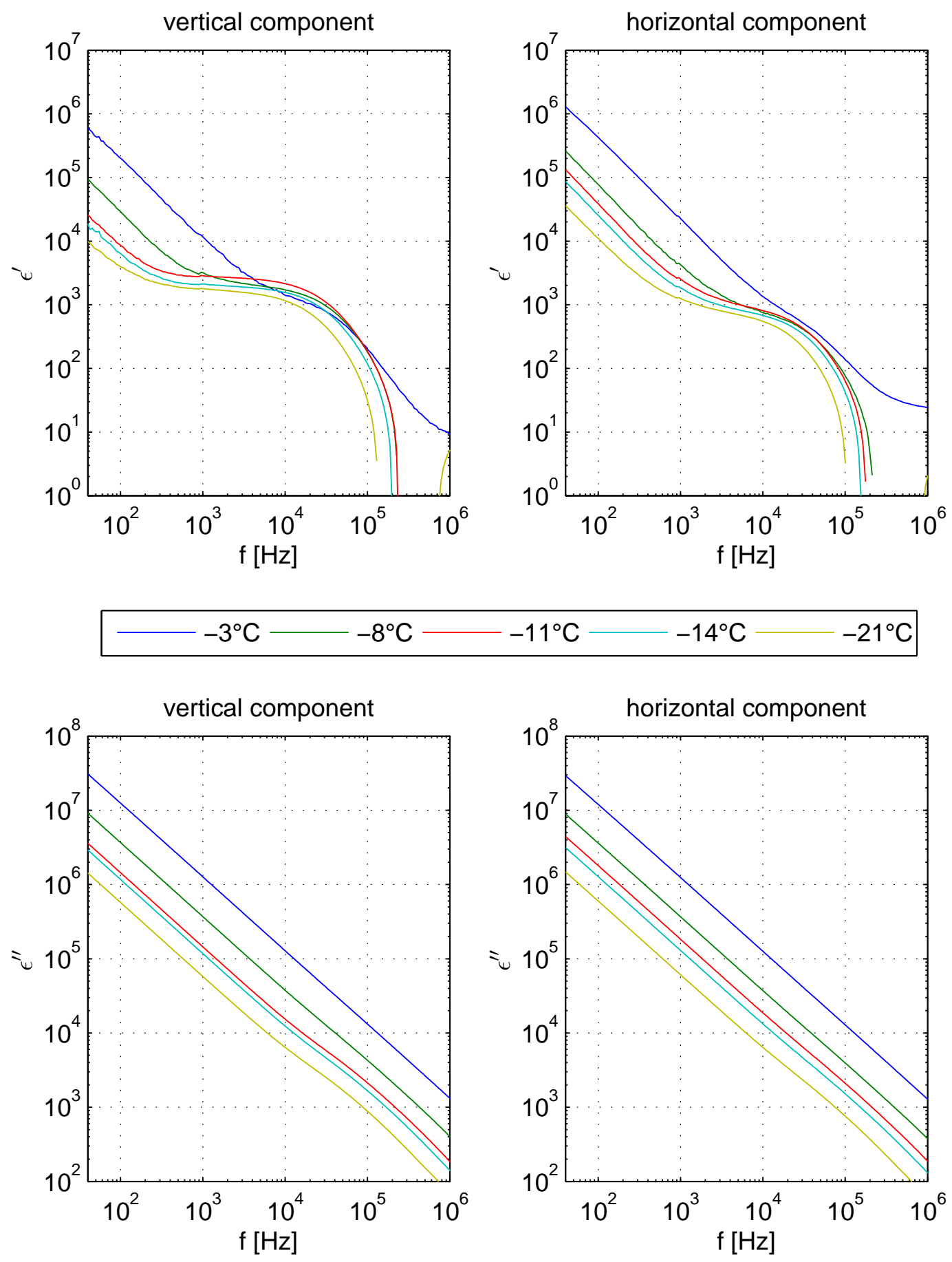

Figure 4.12: Vertical and horizontal components of sea ice permittivity for a four-electrode measurement. 


\subsection{Complex conductivity representation and a broadband physical model}

The discussion at the end of the previous chapter highlighted the limitations of the complex permittivity representation. It was realised that the $1 / w$ factor which transforms the impedance data into a permittivity masks many of the features which appeared strongly in the impedance representation. This limitation remains with the wheelie bin permittivity data as demonstrated by the strong dispersion shown in Figures 4.11 and 4.12. An alternative material property is the complex conductivity $\sigma^{*}$ which is related to the measured impedance by:

$$
Z=\frac{1}{G \sigma^{*}} .
$$

The relationship between complex permittivity and conductivity is:

$$
\sigma^{*}=j \omega \epsilon_{0} \epsilon^{*}
$$

The physical intuition offered by the permittivity representation is not lost. Now, $\sigma^{\prime}$ describes conductive and dielectric loss and $\sigma^{\prime \prime}$ describes polarization. Figures 4.13 to 4.16 show selected sea ice impedance data represented in terms of a complex conductivity. The Argand diagrams shows the data over the same frequency range. 

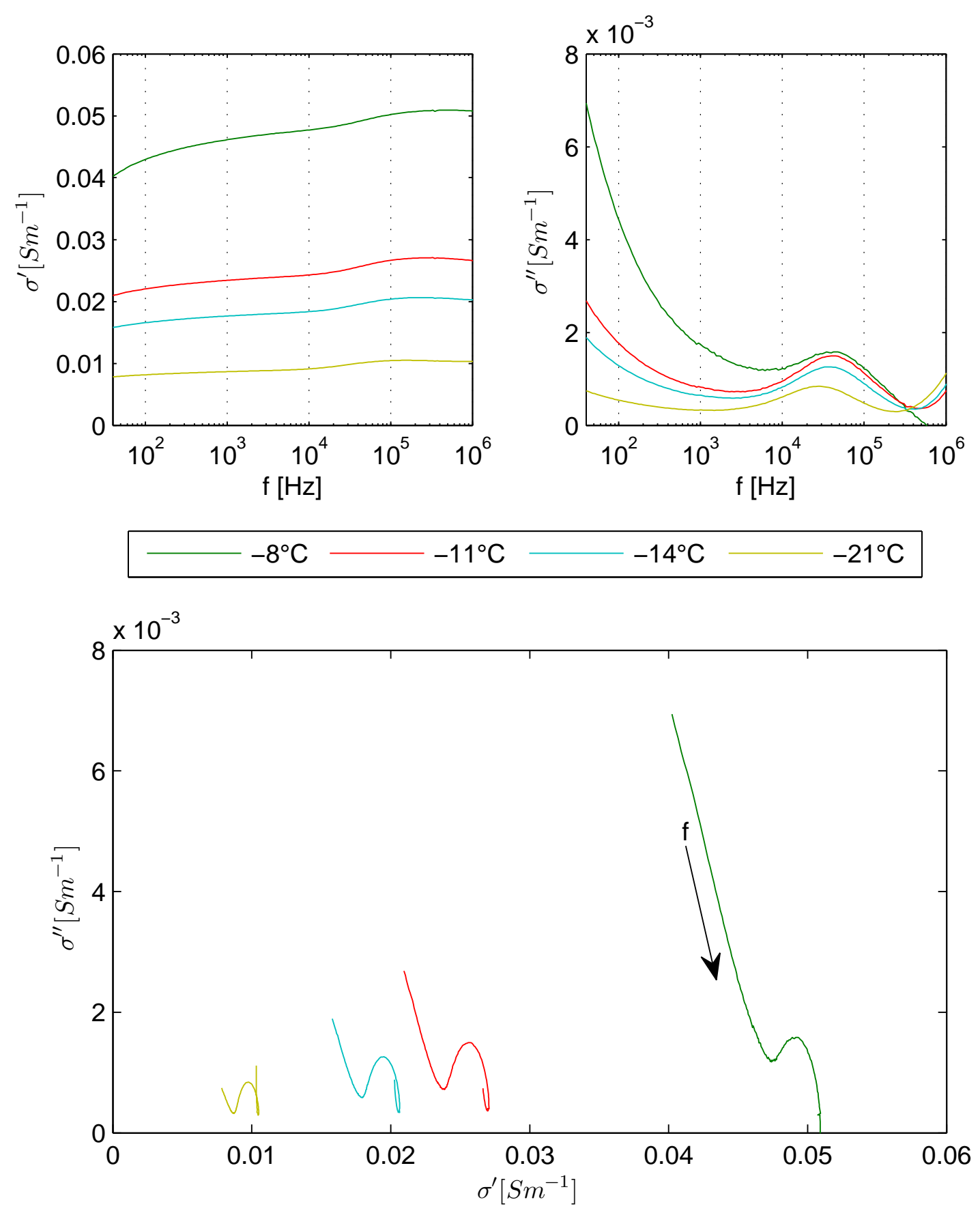

Figure 4.13: Complex conductivity representation of the vertical component of sea ice response for a two-electrode measurement across the electrode pair 1-2. 

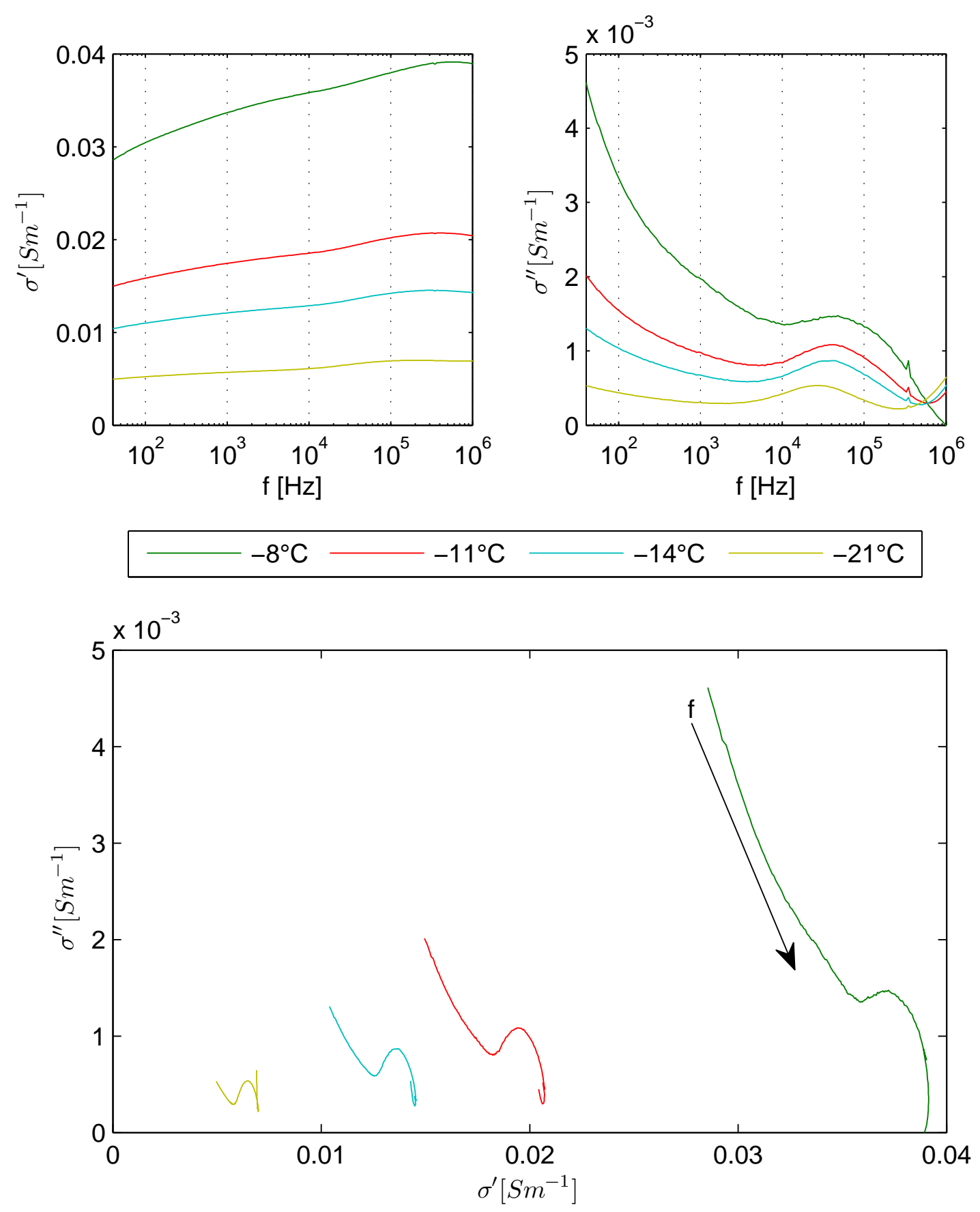

Figure 4.14: Complex conductivity representation of the horizontal component of sea ice response for a two-electrode measurement across the electrode pair 1-2. 

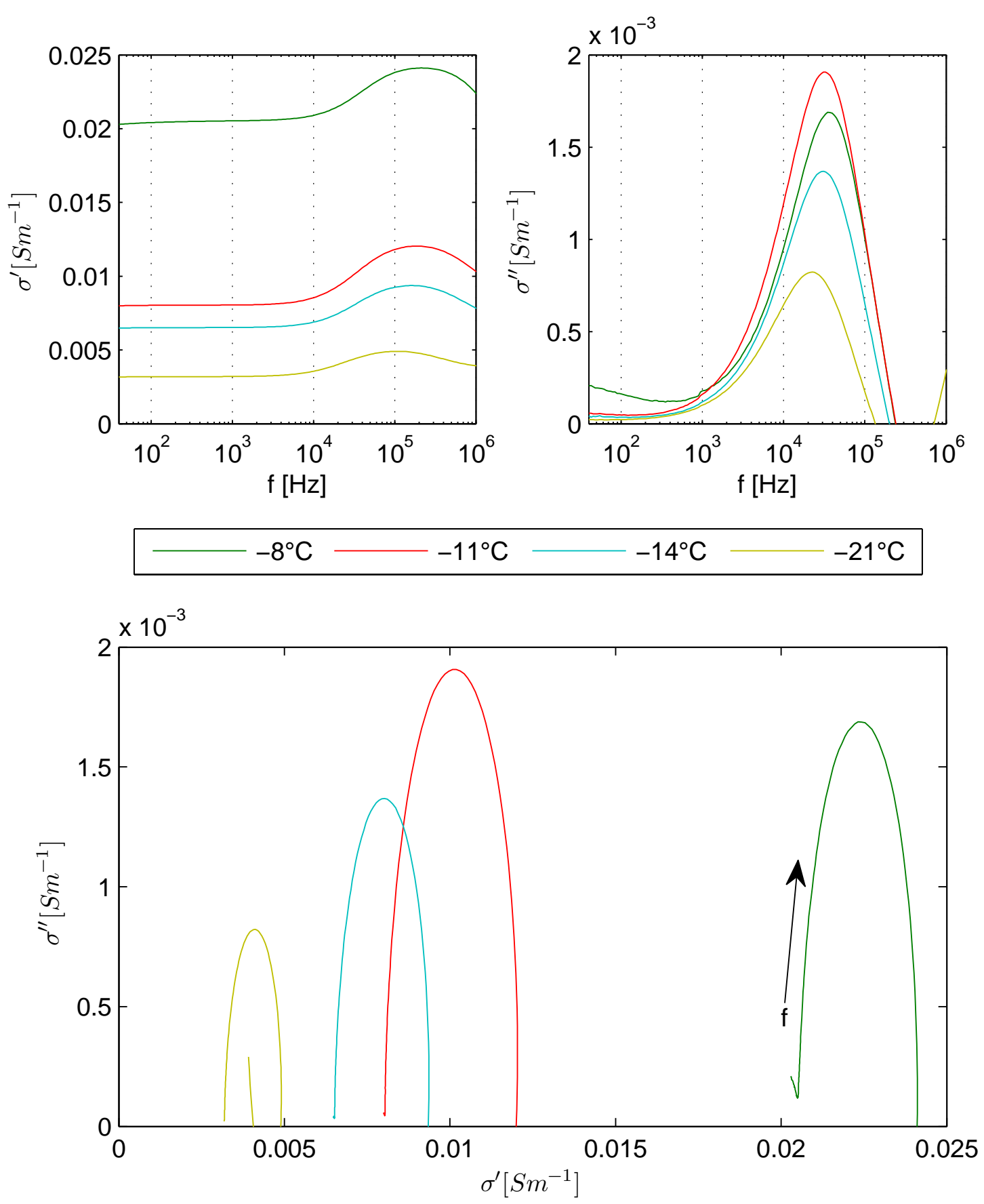

Figure 4.15: Complex conductivity representation of the vertical component of sea ice response for a four-electrode measurement. 

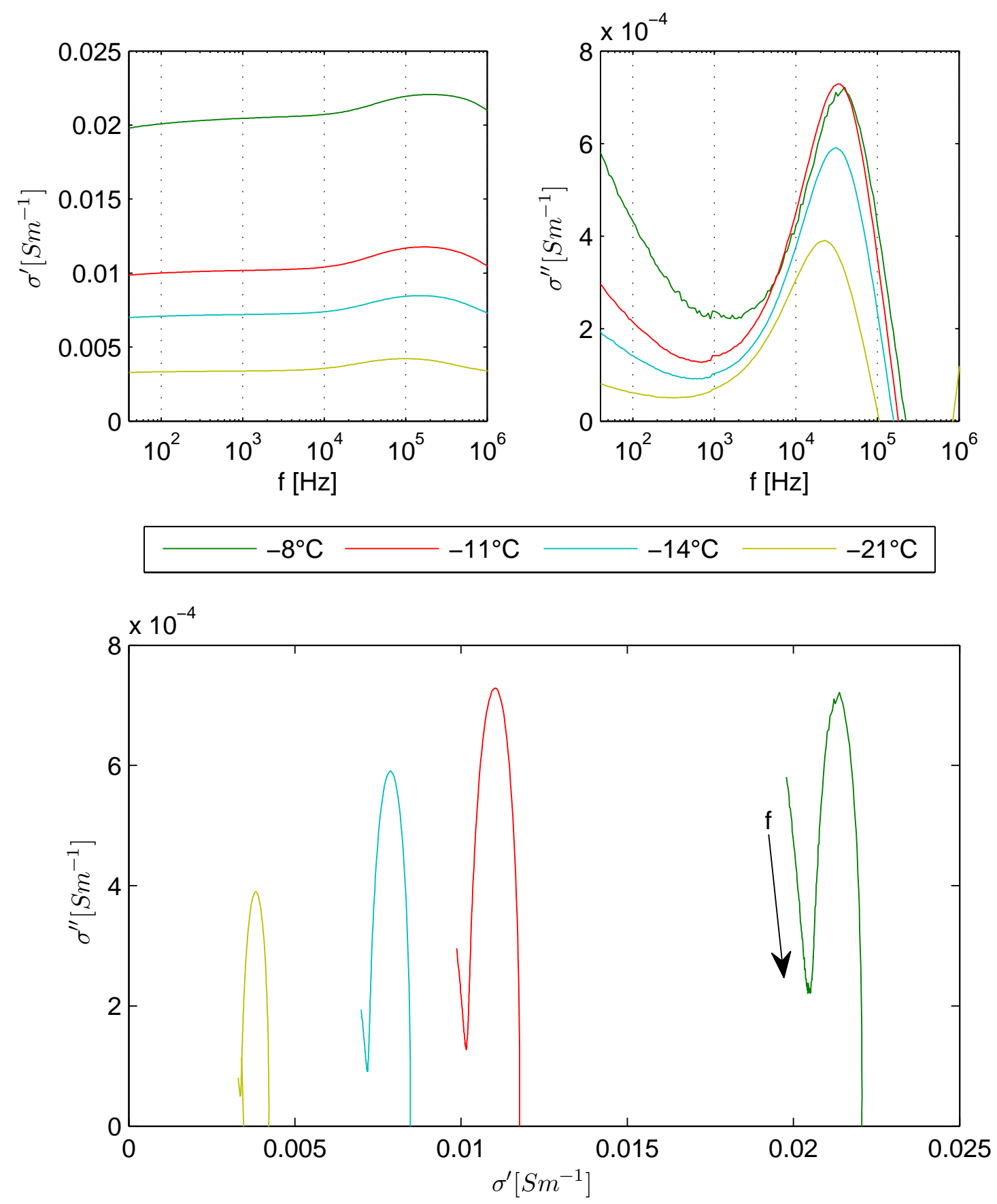

Figure 4.16: Complex conductivity representation of the horizontal component of sea ice response for a four-electrode measurement. 
The complex conductivity representation offers considerable advantages over complex permittivity. Firstly, the dispersive frequency dependence that dominated the permittivity data has been removed, and the data now spans a smaller range and can be visualised on a linear scale. Secondly, when displayed on an Argand diagram, an underlying structure is revealed which is common to all sea ice measurements: The conductivity spectrum is displaced from the origin(1) and it sweeps from a straight line(2) into a semicircle(3). The main advantage of the $\sigma^{*}$ representation is that, these three distinct features correspond to different physical processes. They represent:

1. frequency independent conduction through the bulk material,

2. space charge polarization at internal interfaces, and

3. a Debye relaxation in the ice.

These three processes are incorporated as individual terms (in order) in the following, 6 parameter, model which describes the broadband complex conductivity of sea ice ${ }^{1}$ :

$$
\sigma^{*}=\sigma_{D C}+K(j \omega)^{m}+j \omega \epsilon_{0}\left(\frac{\chi}{1+j \omega \tau}+\epsilon_{\infty}\right)
$$

Alternatively, this model can be expressed in terms of a complex permittivity:

$$
\epsilon^{*}=-j \frac{\sigma_{D C}}{\omega \epsilon_{0}}-\frac{K(j \omega)^{m-1}}{\epsilon_{0}}+\left(\frac{\chi}{1+j \omega \tau}+\epsilon_{\infty}\right)
$$

The usefulness of the model in describing sea ice data is best demonstrated by an example. Figure 4.17 shows a complex conductivity representation of the vertical component of a sea ice impedance measurement.

\footnotetext{
${ }^{1}$ The origin of this model was chaotic. It was, in part, formulated by considering the functions required to reproduce the structure observed in the $\sigma^{*}$ spectrum. This was how the second term was realised and it is entirely empirical. It also incorporates the complex conductivity of a Debye relaxation, which it was realised, produces a semicircle in the $\sigma^{*}$ representation under certain conditions (this peculiarity is outlined in Appendix C).
} 
The data are from the 13th two-electrode measurement across the electrode pair 1-2 and the estimated sample temperature is $-22^{\circ} \mathrm{C}$. A model series generated with parameters ${ }^{2}$ summarised in Figure 4.17 shows good agreement with the experimental data. The coefficient of determination $\mathrm{R}^{2}=0.9998$ demonstrates this. This Figure shows the individual and cumalitive contribution of the three terms in equation 4.8 .

\footnotetext{
${ }^{2}$ The fitting procedure is outlined in section 4.3 .1
} 

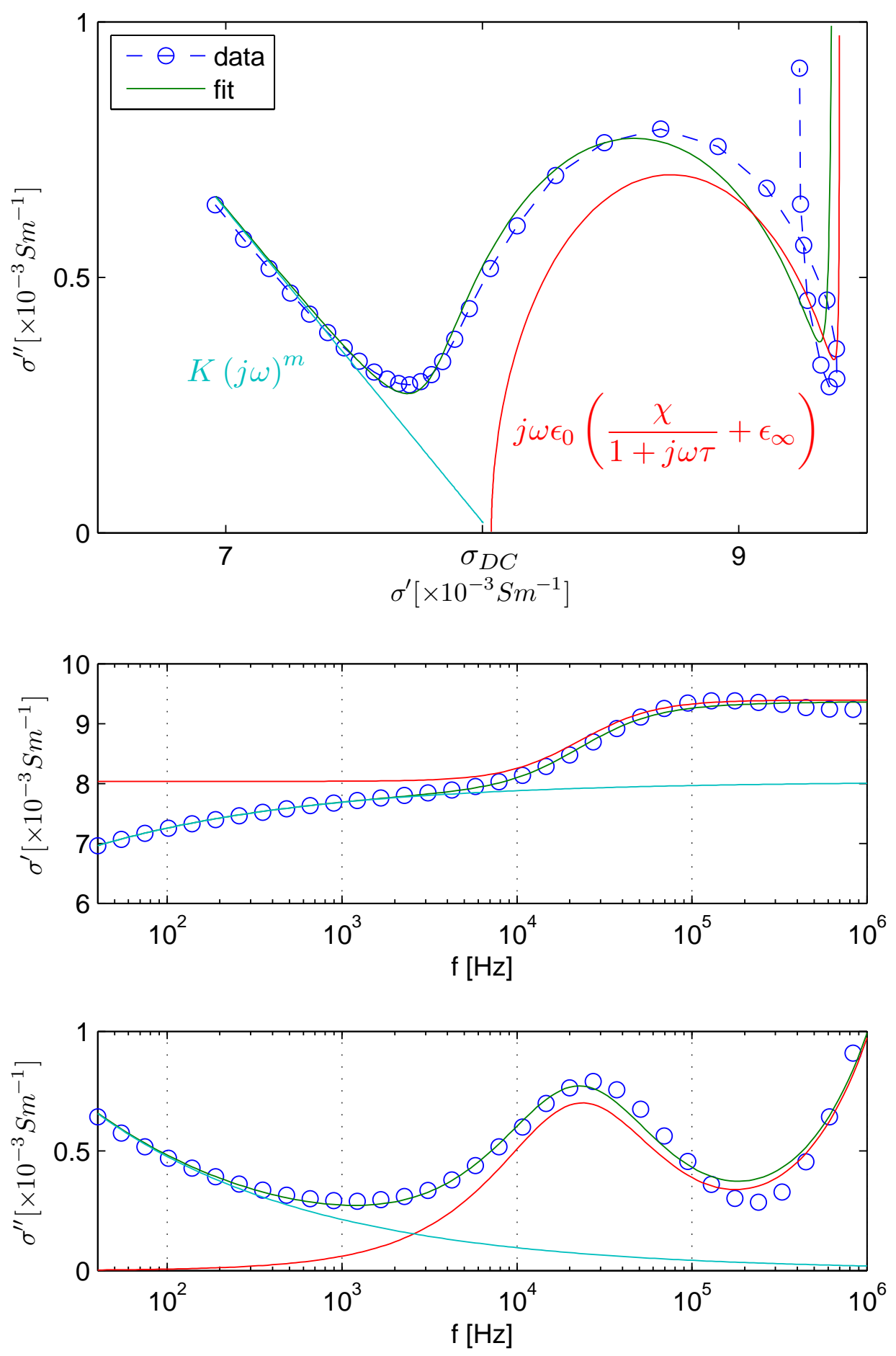

\begin{tabular}{|c|c|c|c|c|c|c|}
\hline $\mathrm{K}\left[\mathrm{Sm}^{-1}\right]$ & $\mathrm{m}$ & $\sigma_{D C}\left[\mathrm{Sm}^{-1}\right]$ & $\chi$ & $\tau\left[\mathrm{s}^{-1}\right]$ & $\epsilon_{\infty}$ & $\mathrm{R}^{2}$ \\
\hline-0.0087 & -0.35 & 0.0080 & 1100 & $7.0 \times 10^{-6}$ & 17 & 0.9998 \\
\hline
\end{tabular}

Figure 4.17: Example of complex conductivity sea ice data represented by broadband physical model. 
The success of the model is that it fully describes the complex (and complicated) conductivity spectrum. However, as alluded to earlier, the three terms in equation 4.8 have clear physical interpretations - this is best realised by representing their contributions as a complex permittivity. Figure 4.18 shows the individual and cumulative contribution of the three terms in equation 4.9 alongside the experimental data.
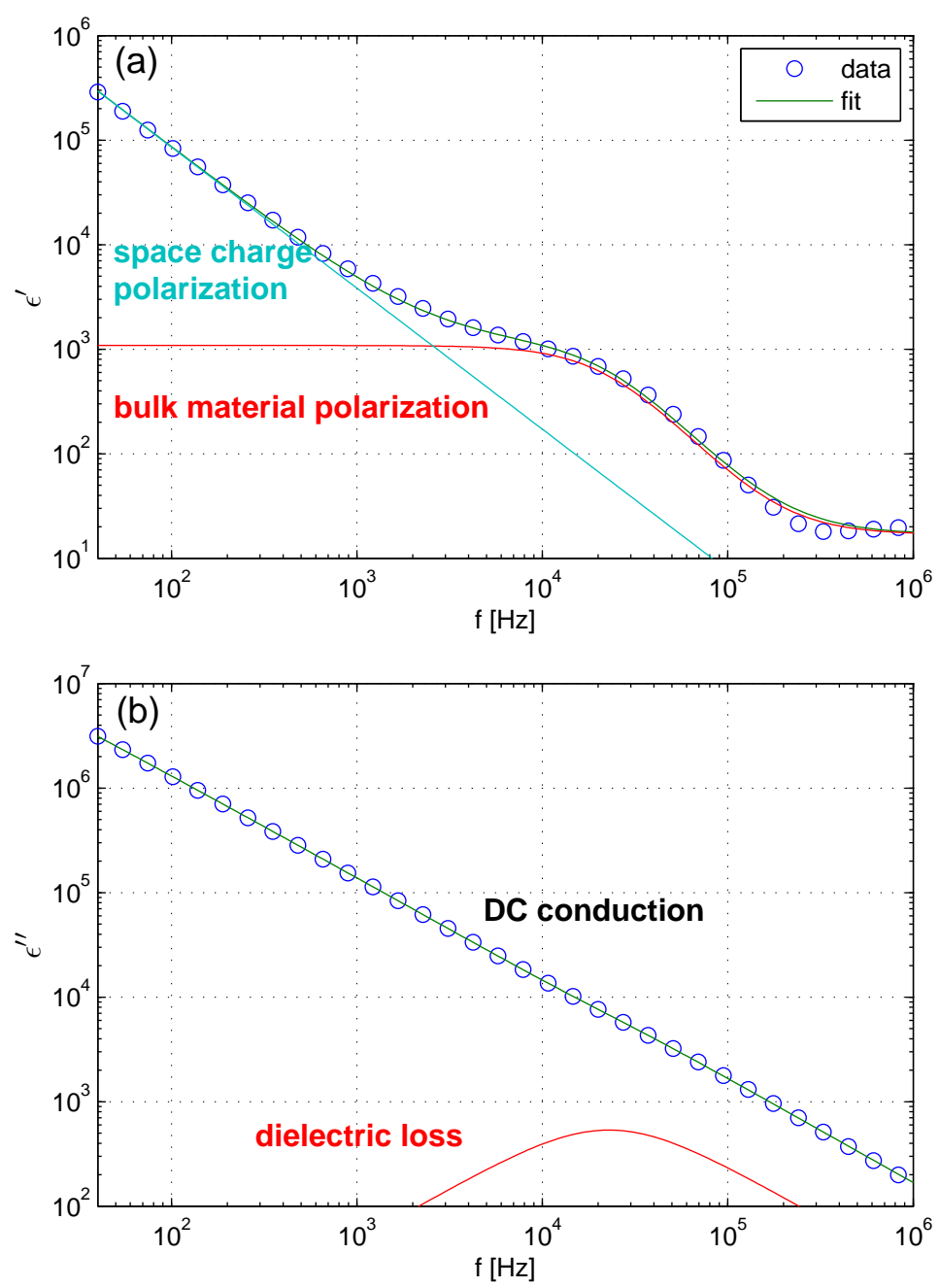

Figure 4.18: Example of complex permittivity sea ice data represented by broadband physical model. 
The real permittivity plot (Figure 4.18(a)), to which only the second and third term of the model contribute, is the most illuminating. The third term underlies the response and the second term in equation 4.9 is additive. The contribution of the second term becomes negligible at high frequencies as $m<0$. However at low frequencies it dominates the response and produces enormous permittivities. These characteristics match the expected contribution of space charge polarization, and we interpret the second term in the model as an empirical description of this phenomenon - it describes the build up of charge at internal interfaces within the sample. It includes electrode polarization for a two-electrode measurement.

If interfacial polarization is accounted for, the underlying response must describe the bulk polarization of this material. The material evidently undergoes a Debye relaxation. Pure ice exhibits a dielectric relaxation at a similar frequency, which suggests that the relaxation described by the third term in equation 4.8 is that of ice within the medium. This interpretation will be confirmed through further analysis in section 4.3.2.

The meaning of $\sigma_{D C}$ is then clear. It is the real conductivity that would be measured if the space charge polarization and bulk polarization processes were absent. This is the condition of a DC measurement and therefore $\sigma_{D C}$ then represents the frequency independent conduction through the bulk material.

The complex conductivity representation and the physical interpretations of the individual terms are summarised in Table 4.3. 


\begin{tabular}{|c|c|l|}
\hline$\sigma^{*}$ & $\epsilon^{*}$ & Physical interpretation \\
\hline$\sigma_{D C}$ & $-j \frac{\sigma_{D C}}{\omega \epsilon_{0}}$ & $\begin{array}{l}\text { Frequency independent } \\
\text { conduction through the } \\
\text { bulk material. }\end{array}$ \\
\hline$K(j \omega)^{m}$ & $-\frac{K(j \omega)^{m-1}}{\epsilon_{0}}$ & Space charge polarization. \\
\hline$j \omega \epsilon_{0}\left(\frac{\chi}{1+j \omega \tau}+\epsilon_{\infty}\right)$ & $\left(\frac{\chi}{1+j \omega \tau}+\epsilon_{\infty}\right)$ & $\begin{array}{l}\text { Bulk material polarization } \\
\text { and dielectric loss. The } \\
\text { material undergoes a De- } \\
\text { bye relaxation. }\end{array}$ \\
\hline
\end{tabular}

Table 4.3: A summary of this physical interpretation. The text colours match the curves in the above plots. 


\subsubsection{Fitting procedure}

The model parameters in the table in Figure 4.17 were obtained by performing a non-linear least squares fit (CNLSF) on the complex conductivity data to converge it to equation 4.8. This process involves iterative steps from some initial conditions to a solution which minimises the square of the complex fit residuals. The fit was performed in MATLAB and the code and underlying theory is outlined in Appendix D. Here, some comments on fit settings and the usability and performance of the script are made.

For the four-electrode complex conductivity data, the general structure, shown in Figure 4.17 and represented by equation 4.8, became apparent as soon as the sensor became enclosed in ice. For the twoelectrode measurements, electrode polarization (incorporated in term 2) obscured bulk polarization (term 3 ) down to a temperature of $-8^{\circ} \mathrm{C}$. The fit was achievable as soon as this structure became apparent.

The curve fitting script was robust and could achieve convergence from some quite general initial parameters. With suitable initial conditions it took approximately 20 iterations for the solution to converge. The fit parameters which varied the most across the course of the experiment were $\sigma_{D C}$ and $K$, and sometimes a custom initial setting was required to achieve convergence for a given conductivity measurement. The typical initial parameter settings are summarised in Table 4.4. It was generally possible to run the script in a loop to perform the fit for $\sigma^{*}$ data for the entire temperature range for a given electrode combination.

\begin{tabular}{|c|c|c|c|c|c|}
\hline$K\left[\mathrm{Sm}^{-1}\right]$ & $m$ & $\sigma_{D C}\left[\mathrm{Sm}^{-1}\right]$ & $\chi$ & $\tau\left[\mathrm{s}^{-1}\right]$ & $\epsilon_{\infty}$ \\
\hline-0.01 & -0.4 & 0.01 & 1000 & $5 \times 10^{-6}$ & 3 \\
\hline
\end{tabular}

Table 4.4: Initial parameters for sea ice complex non-linear least squares fitting.

The fit was performed on all sea ice data (two-electrode and four- 
electrode) across the frequency range $40 \mathrm{~Hz}-100 \mathrm{kHz}$. This range includes the dielectric relaxation and largely excludes the high frequency measurement error. The high frequency measurement error still existed in the fourelectrode data at the upper limit of this range and it adversely affected the recovery of $\epsilon_{\infty}$. The solution sometimes included negative values of $\epsilon_{\infty}$ which is nonsensical. For these cases, this parameter was ignored. It is believed that, because it occurred only at the upper frequency limit of the data series, the error only adversely affected the recovery of $\epsilon_{\infty}$.

The coefficient of determination of the solutions was typically of the order of $R^{2}=1$. A more rigorous fitting procedure would calculate confidence intervals of the fit parameters. This is not something we invested time in. Instead, some intuition was gained into the error of the fit parameters $\chi$ and $\tau$ by examining Figure 4.17. The relaxation frequency $(1 / 2 \pi \tau)$ is easily identifiable on the plot - it is the frequency corresponding to the mid-frequency bump in $\sigma^{\prime \prime}$. The parameter $\chi$ is related to the magnitude of this bump $\sigma_{\text {bump }}^{\prime \prime}$ by the equation ${ }^{3} \chi=2 \tau \sigma_{\text {bump }}^{\prime \prime} / \epsilon_{0}$. In this manner, the parameters $\chi$ and $\tau$ were extracted from the plot. These estimates were compared with the fit parameters and the fit errors were estimated from the difference between the two techniques. Fit parameter errors of $\Delta \tau / \tau=0.2$ and $\Delta \chi / \chi=0.2$ were estimated.

Each complex conductivity data series comprised a real and imaginary number at the 127 measurement frequencies. These 254 numbers provide a high resolution description of the broadband material response between $40 \mathrm{~Hz}$ and $100 \mathrm{kHz}$. The achievement of this model and the fitting procedure is that the material response, across the same frequency range, can be described with six parameters, and with little loss of resolution. The model is applicable to sea ice across a large temperature range. The aim of this thesis, to determine how the electrical response of sea ice depends on microstructure, requires an understanding of how these five parameters

\footnotetext{
${ }^{3}$ This follows from evaluating equation 4.8 at the relaxation frequency and holds provided $\epsilon_{\infty}$ is small.
} 
depend on microstructure. A sea ice microstructure monitoring system would track how they vary with time and temperature.

The wheelie bin experiment produced a simple microstructure and the complex conductivity along two projections was monitored. Any variation in the fit parameters between the horizontal and vertical capacitors would indicate a sensitivity to brine microstructure. In the following sections, the fit parameters describing the horizontal and vertical components of the complex conductivity data, and their variation with temperature, are examined.

\subsubsection{Bulk material processes}

Bulk material polarization and conduction are described by the equation:

$$
\sigma^{*}=\sigma_{D C}+j \omega \epsilon_{0}\left(\frac{\chi}{1+j \omega \tau}+\epsilon_{\infty}\right)
$$

The expression includes a Debye equation indicating that the material undergoes a dielectric relaxation. When this physical model was introduced it was alluded that this relaxation occurred within the ice phase. This was confirmed by analysis of the temperature dependence of the relaxation time $\tau$.

Figure 4.19 shows the relaxation time of the sea ice medium vs temperature. The relationship between relaxation time and relaxation frequency is $f_{\text {relax }}=1 / 2 \pi \tau$. The solid line at the top of the plot is an experimental result [Evans (1965)] of the relaxation time of a pure ice sample. The plot shows that sea ice undergoes relaxation at a considerably higher frequency than pure ice.

Jaccard $(1959,1964)$ theory suggests that the relaxation time should follow a Boltzmann distribution. The $1 / T$ vs $\log \tau$ axes were chosen to demonstrate this. The relationship between the activation energy $E_{a}$ of the polarization mechanism, temperature and the relaxation time is:

$$
\tau=\tau_{0} \exp \left(\frac{E_{a}}{k_{B} T}\right)
$$




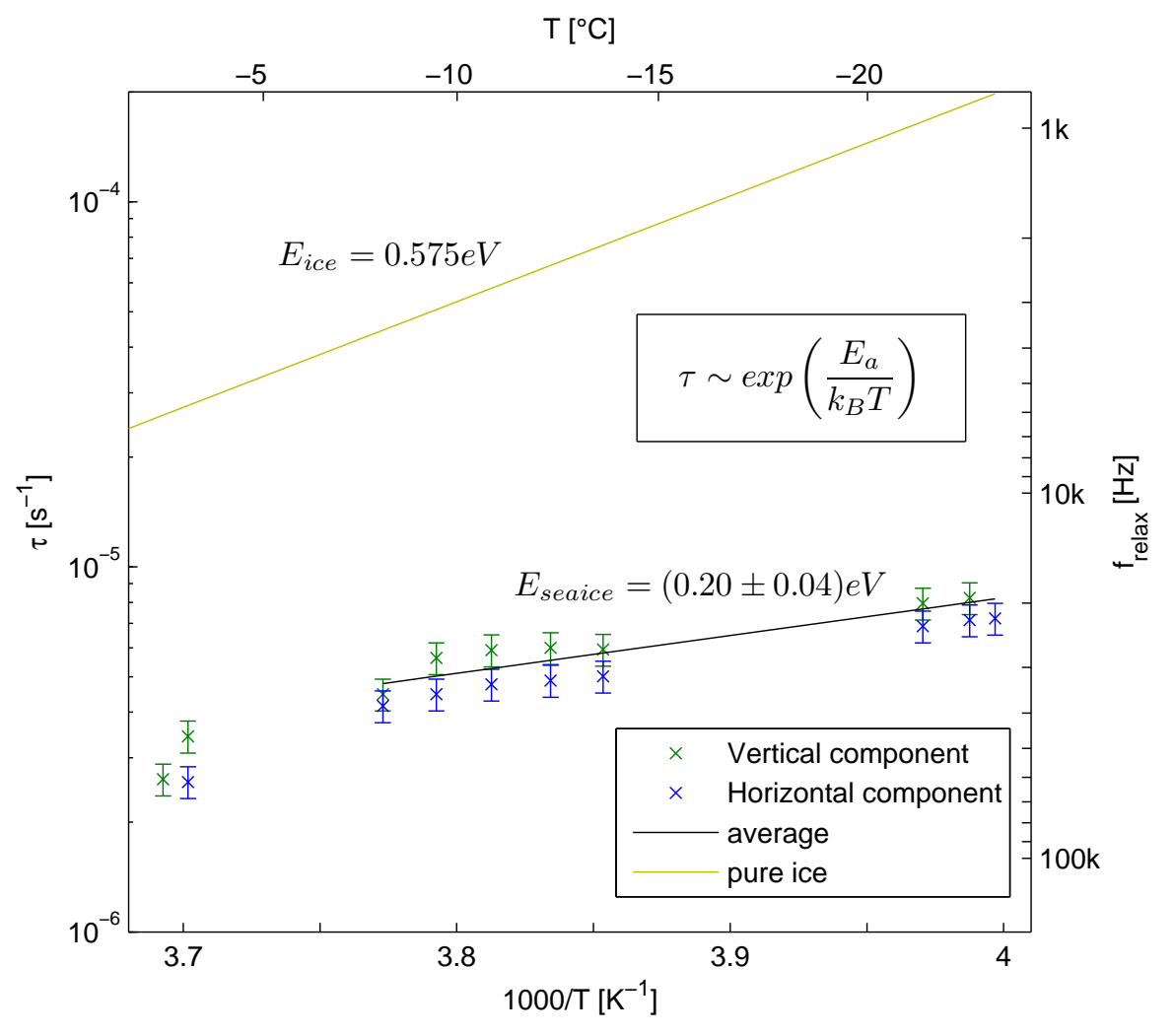

Figure 4.19: The relaxation time of sea ice vs temperature. The relationship obeys a Boltzmann distribution.

A straight line linear least-squares fit performed on the experimental data yielded parameters; $E_{a}=0.200 \pm 0.004[\mathrm{eV}]$ and $\tau_{0}=(6 \pm 1) \times 10^{-10}\left[\mathrm{~s}^{-1}\right]$. These quantities are the average of the vertical and horizontal components - there is little difference between the two projections. However, this activation energy does not reflect the uncertainty of $20 \%$ in the raw $\tau$ data. Taking this into account gave an estimate of $E_{a}=0.20 \pm 0.04[\mathrm{eV}]$.

Experimental work by Jaccard (1959), Takei and Maeno (1987), Addison (1970) and Grimm et al. (2008) demonstrates that the addition of impurities can drastically decrease the relaxation time and activation energy of ice. Grimm et al. (2008) present a study of the relaxation time of $\mathrm{NaCl}$ doped 
ice over a large temperature range and for varying salt concentrations. Their measurements were performed at much lower temperatures than ours, to achieve inconsequentially low brine volume fractions - making the Debye relaxation easily identifiable above space charge effects. However, if their data are extrapolated to our temperature range, it shows excellent agreement with our experimental sea ice values. They find that for high salt concentrations the ice lattice becomes saturated (can incorporate no more impurities) at an activation energy of $\sim 0.29 \mathrm{eV}$. This value is in excellent agreement with this sea ice experiment. This agreement confirms that the relaxation observed is that of a doped ice sample.

The susceptibility $\chi$, or magnitude of this relaxation, is shown in Figures 4.20(b) and 4.20(d) for two-electrode and four-electrode measurements. The uncertainty in this parameter is $\Delta \chi / \chi=0.2$. The parameter is strongly anisotropic with the vertical component between two and three larger than the horizontal. The measured susceptibility is also much larger than the $\sim 100$ that it is for a pure ice sample.

Figures 4.20(a) and 4.20(c) show the DC conductivity $\sigma_{D C}$ for the same measurements. The parameter is anisotropic for the two-electrode measurement, with the vertical component being larger than the horizontal component, and isotropic for the four-electrode measurement. 

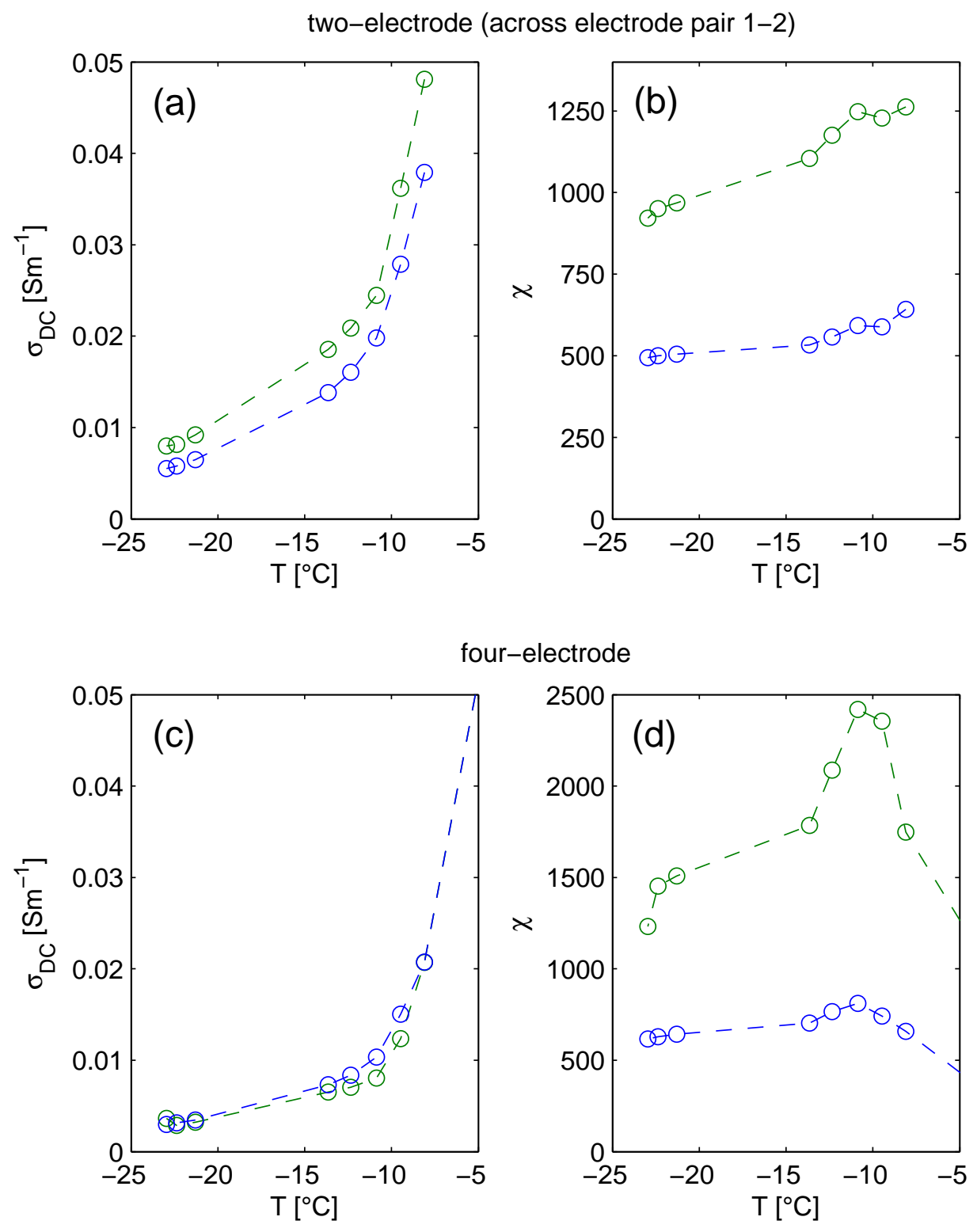

$-\ominus-$ Vertical component $-\ominus-$ Horizontal Component

Figure 4.20: The evolution with temperature of the fit parameters $\sigma_{D C}$ (a),(c) and $\chi(\mathrm{b}),(\mathrm{d})$ for different capacitor orientations and different measurement methods. 
In order to measure the true effective material properties then the volume of material sampled should be much larger than the microstructual features. For the two-electrode measurement across electrode pair 1-2 this is not the case. Figure 4.21 shows the cell electrodes superimposed on the observed sea ice microstructure. Figure 4.21(a) shows that the

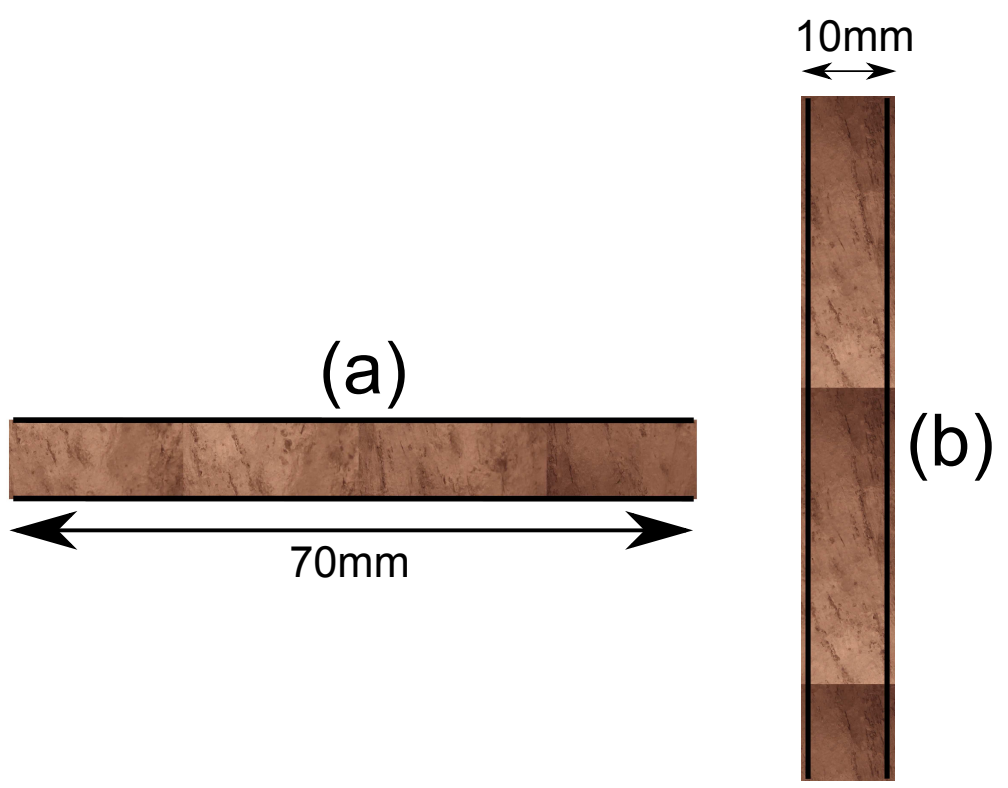

Figure 4.21: Cell electrodes $1 \& 2$ superimposed on the observed sea ice microstructure for the two different cell orientations. The microstructure image has been repeated to fill the entire capacitor.

long axis of the elongated brine pockets is comparable to the electrode spacing whereas Figure 4.21(b) shows that the short axis is not. There are likely brine channels which link the two electrodes of the horizontally orientated capacitor, providing an electrical short circuit and increasing the DC conductivity. The four-electrode measurement samples a medium approximately three times as wide and there are unlikely to be brine pockets which span the electrodes. This could explain why conductance anisotropy was observed only for the 1-2 two-electrode measurements. If the brine pockets are small compared to the volume sampled then their 
orientation does not appear to strongly influence the DC conductivity of the medium.

The DC conductivity was compared to experimental data for Arctic sea ice to determine how realistic the Wheelie bin sea ice was. At $-10^{\circ} \mathrm{C}$, Ingham et al. (2008) give a DC conductivity for Barrow, Alaska sea ice of $0.001\left[\mathrm{Sm}^{-1}\right]$. This is approximately a factor of 10 lower than the fourelectrode data. This suggests that the Wheelie bin sea ice is of higher salinity than natural sea ice.

The high frequency permittivity $\epsilon_{\infty}$ describes the magnitude of the polarization at frequencies above that of ice relaxation. The parameter could only be recovered accurately for the 1-2 two-electrode measurements in which the impedance measurement error was less prominent. The variation with temperature of the vertical and horizontal component is shown in Figure 4.22. The parameter varies between 60 and 5 across the temperature range. It exhibits anisotropy and at low temperatures the vertical component is greater than the horizontal. 


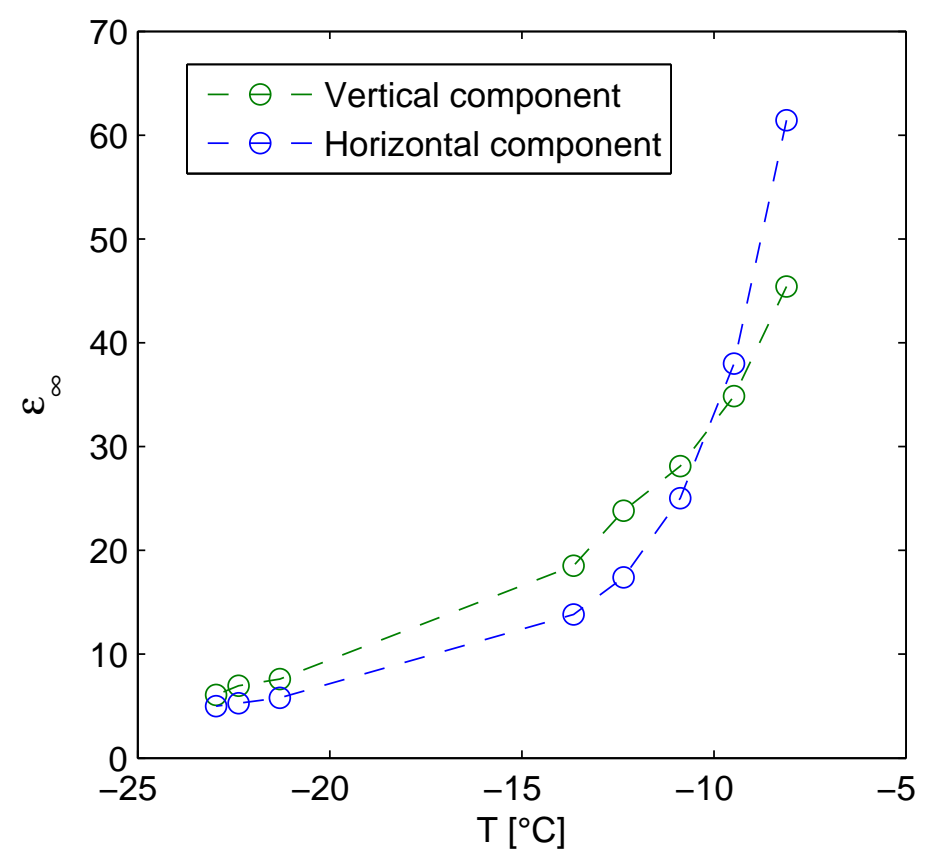

Figure 4.22: The high frequency permittivity as measured across the twoelectrode pair 1-2. 


\subsubsection{Interfacial polarization}

The broadband physical model distinguishes interfacial polarization from bulk material processes. Interfacial polarization, or space charge polarization, includes the parasitic affect of electrode polarization and internal space charge polarization. Electrode polarization occurs where the material interfaces with the electrodes, and internal space charge polarization occurs at ice/brine interfaces within the material. These are low frequency processes described by the empirical term $K(j \omega)^{m}$ in equation 4.8.

The imaginary component of the term describes polarization and the real part describes conduction. The real part is negative, indicating the process reduces the conductivity of the material. This is physical - if a material has a lot of interfaces that block the flow of current then it is going to be less conductive.

Figure 4.23 shows the evolution with temperature of the parameters $K$ and $m$. The parameter $K$ is the better of the two for comparing the relative magnitude of the affect. The larger (the more negative) this parameter is the larger the space charge polarization contribution. The magnitude of $K$ decreases with decreasing temperature as both the brine volume fraction, and the area of polarizable interfaces, decreases. The parameter is larger for two-electrode measurements than four-electrode measurements indicating electrode polarization plays a dominating role in the two-electrode measurement.

This parameter indicates strong anisotropy. For the 1-2 two-electrode measurement, the vertical exceeds the horizontal component. For a fourelectrode measurement, the horizontal exceeds the vertical component. 

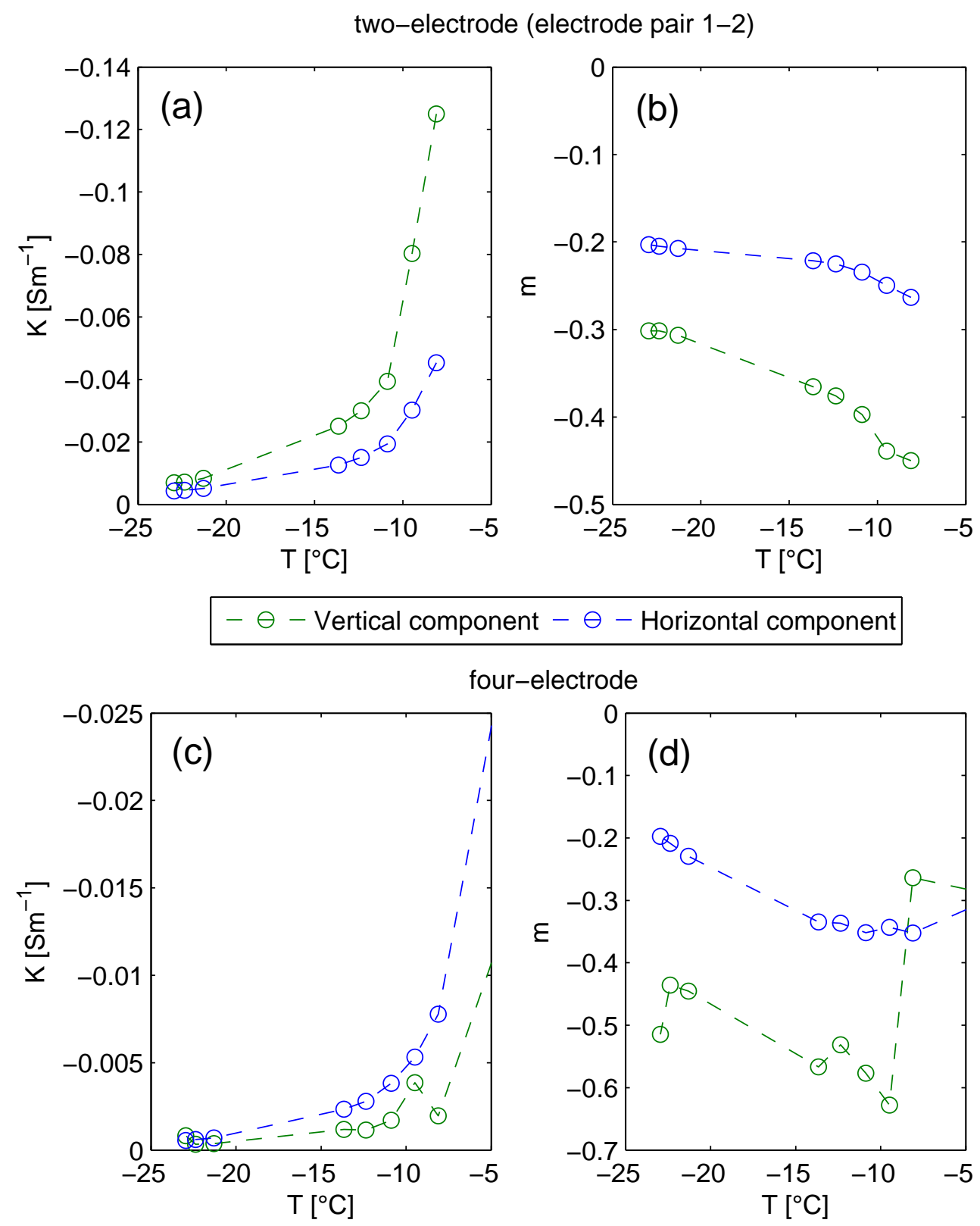

Figure 4.23: The evolution with temperature of the parameters $K(\mathrm{a}, \mathrm{c})$ and $m(\mathrm{~b}, \mathrm{~d})$ which describe the process of space charge polarization. 


\subsection{Mixture model}

The analytical procedure described in the previous section distinguished bulk material polarization and conduction data. Study of the variation with temperature of the horizontal and vertical components of the bulk material properties $\sigma_{D C}, \chi$ and $\epsilon_{\infty}$ revealed that:

- The bulk conductivity $\sigma_{D C}$ for the four-electrode measurement showed no evidence of anisotropy. This is surprising as there is experimental evidence [Jones et al. (2009)] that the electrical conductivity of real sea ice is greater in the vertical direction than in the horizontal.

- The susceptibility $\chi$ was always much larger than the approximately 100 that it is for ice in isolation. It exhibits strong anisotropy the vertical component of the susceptibility was approximately $2 \times$ larger than the horizontal component.

- The high frequency permittivity was similarly anisotropic.

We attempted to reconcile these results with the dielectric mixture theory outlined in section 2.4. The susceptibility, high frequency permittivity and DC conductivity were modelled for a dispersed phase of conducting dielectric ellipsoids (brine pockets) within a conductive dielectric medium (ice). The ellipsoids lie at an angle $\theta$ from the vertical and their azimuthal orientation is random as depicted in Figure 4.24. This is the 'VTS' model which has previously been applied to sea ice data at high frequencies (50MHz and above). We adapted this model to calculate the horizontal and vertical components of the effective material properties at frequencies above and below the relaxation of ice.

In the 'VTS' model, the electrical properties of the dispersed and background phase are represented by complex permittivities $\epsilon_{b r i n e}^{*}$ and $\epsilon_{i c e}^{*}$ respectively. In order to calculate the effective properties for the advanced geometry depicted in Figure 4.24, Tinga's equations must first be evaluated for media with ellipsoids aligned parallel and perpendicular 


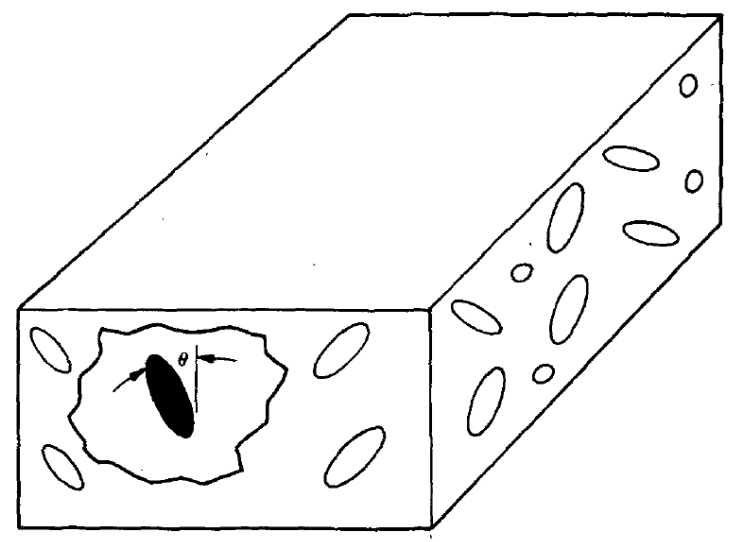

Figure 4.24: Model of sea ice microstructure from Vant et al. (1978).

to the polarizing field. For ellipsoids with long axis aligned parallel to the field, the effective complex permittivity is:

$$
\epsilon_{\|}^{*}=\epsilon_{i c e}^{*}\left(1+\frac{V_{2}}{V_{1}} \frac{\epsilon_{b r i n e}^{*}-\epsilon_{i c e}^{*}}{\left[\epsilon_{i c e}^{*}+n_{\|}\left(\epsilon_{\text {brine }}^{*}-\epsilon_{i c e}^{*}\right)\left(1-V_{2} / V_{1}\right)\right]}\right)
$$

where the depolarization ratio is:

$$
n_{\|}=\left(\frac{b}{a}\right)^{2}\left(\frac{1}{2 e^{3}}\right)\left(-2 e+\ln \frac{1+e}{1-e}\right) .
$$

The ellipsoid eccentricity, $e=\left[1-\left(\frac{b}{a}\right)^{2}\right]^{\frac{1}{2}}$. For ellipsoids with long axis aligned perpendicular to the field, the effective complex permittivity is:

$$
\epsilon_{\perp}^{*}=\epsilon_{i c e}^{*}\left(1+\frac{V_{2}}{V_{1}} \frac{\epsilon_{b r i n e}^{*}-\epsilon_{i c e}^{*}}{\left[\epsilon_{i c e}^{*}+n_{\perp}\left(\epsilon_{\text {brine }}^{*}-\epsilon_{i c e}^{*}\right)\left(1-V_{2} / V_{1}\right)\right]}\right)
$$

where the depolarization ratio is:

$$
n_{\perp}=\left(\frac{b}{a}\right)^{2}\left(\frac{1}{4 e^{3}}\right)\left(\frac{2 e}{(b / a)^{2}}+\ln \frac{1-e}{1+e}\right) .
$$


The real component of the effective permittivity for ellipsoids with long axis aligned at an angle $\theta^{\prime}$ from the electric field is:

$$
\begin{aligned}
\epsilon^{\prime}\left(\theta^{\prime}\right) & =\left|\left(\begin{array}{ccc}
\epsilon_{\perp}^{\prime} & 0 & 0 \\
0 & \epsilon_{\perp}^{\prime} & 0 \\
0 & 0 & \epsilon_{\|}^{\prime}
\end{array}\right)\left(\begin{array}{c}
\sin \theta^{\prime} \\
\sin \theta^{\prime} \\
\cos \theta^{\prime}
\end{array}\right)\right| \\
& =\sqrt{\epsilon_{\perp}^{\prime 2} \sin ^{2} \theta^{\prime}+\epsilon_{\|}^{\prime 2} \cos ^{2} \theta^{\prime}} .
\end{aligned}
$$

The equivalent expression for the imaginary component is:

$$
\begin{aligned}
\epsilon^{\prime \prime}\left(\theta^{\prime}\right) & =\left|\left(\begin{array}{ccc}
\epsilon_{\perp}^{\prime \prime} & 0 & 0 \\
0 & \epsilon_{\perp}^{\prime \prime} & 0 \\
0 & 0 & \epsilon_{\|}^{\prime \prime}
\end{array}\right)\left(\begin{array}{c}
\sin \theta^{\prime} \\
\sin \theta^{\prime} \\
\cos \theta^{\prime}
\end{array}\right)\right| \\
& =j \sqrt{\epsilon_{\perp}^{\prime \prime 2} \sin ^{2} \theta^{\prime}+\epsilon_{\|}^{\prime \prime 2} \cos ^{2} \theta^{\prime}} .
\end{aligned}
$$

For the geometry of Figure 4.24, for vertical fields $\theta^{\prime}=\theta$ and the vertical component of the effective permittivity is:

$$
\epsilon_{V}^{*}=\sqrt{\epsilon_{\perp}^{\prime 2}+\left(\epsilon_{\|}^{\prime 2}-\epsilon_{\perp}^{\prime 2}\right) \cos ^{2} \theta}+j \sqrt{\epsilon_{\perp}^{\prime \prime 2}+\left(\epsilon_{\|}^{\prime \prime 2}-\epsilon_{\perp}^{\prime \prime 2}\right) \cos ^{2} \theta} .
$$

For horizontal fields $\cos \theta^{\prime}=\sin \theta \cos \phi$, where $\phi$ is the azimuthal orientation. In the model medium, the ellipsoids have random azimuthal orientation and $\cos \phi$ averages to $\frac{1}{2}$. The horizontal component of the effective permittivity is then:

$$
\epsilon_{H}^{*}=\sqrt{\epsilon_{\perp}^{\prime 2}+\frac{1}{2}\left(\epsilon_{\|}^{\prime 2}-\epsilon_{\perp}^{\prime 2}\right) \sin ^{2} \theta}+j \sqrt{\epsilon_{\perp}^{\prime \prime 2}+\frac{1}{2}\left(\epsilon_{\|}^{\prime \prime 2}-\epsilon_{\perp}^{\prime \prime 2}\right) \sin ^{2} \theta} .
$$

The microstructual inputs of the model are; the aspect ratio $a / b$, the brine volume fraction $V_{2} / V_{1}$ and the angle $\theta$ by which the ellipsoids are tilted from the vertical. The material properties of the two phases must also be specified. The conductivity and dielectric constant of brine in sea ice are independent of frequency across this range and are given by Stogryn and Desargant (1985).

$$
\sigma_{\text {brine }}= \begin{cases}-T \exp (0.5193+0.08755 T) & \text { for } T \geq-22.9 \\ -T \exp (1.0334+0.1100 T) & \text { for } T<-22.9\end{cases}
$$




$$
\epsilon_{\text {brine }}^{*}=\frac{939.66-19.068 T}{10.737-T}-j \frac{\sigma_{\text {brine }}}{\epsilon_{0} \omega}
$$

The input value for the DC conductivity of the ice phase is unclear due to it's strong dependence on ice impurities and $\sigma_{D C}$ was treated as a temperature independent model input. Evans (1965) gives the dielectric constant of pure ice and it was assumed that the doped ice of sea ice is similar.

$$
\begin{aligned}
\sigma_{D C . i c e} & =? \\
\epsilon_{D C . i c e}^{*} & =\frac{20715}{T+273-38}-j \frac{\sigma_{D C . i c e}}{\epsilon_{0} \omega}
\end{aligned}
$$

In the high frequency limit the permittivity of pure ice is:

$$
\epsilon_{\infty . i c e}^{*}=3.14-j \frac{\sigma_{D C . i c e}}{\epsilon_{0} \omega} .
$$

The cascade of equations outlined above were evaluated to yield the horizontal and vertical components of the effective permittivity in the low and high frequency limit. The low frequency computation was performed at $1 \mathrm{~Hz}$ and the high frequency computation at $100 \mathrm{MHz}$. The real component of the high frequency permittivity is the model output $\epsilon_{\infty}$. We decoupled the susceptibility and DC conductivity from the high and low frequency complex permitivities for horizontal and vertical projections using the equations:

$$
\begin{aligned}
\chi & =\epsilon_{D C}^{\prime}-\epsilon_{\infty}^{\prime} \\
\sigma_{D C} & =\epsilon_{0} \omega \epsilon_{D C}^{\prime \prime}
\end{aligned}
$$

Model data was generated for a simple microstructural evolution between temperatures of $-5^{\circ} \mathrm{C}$ and $-25^{\circ} \mathrm{C}$. The aspect ratio of the ellipsoids was made constant and the brine volume fraction decreased with temperature whilst constrained by the constant salinity of the sea ice. The equations of Cox and Weeks (1983) and Lepparanta and Manninen (1988) provide the link between the state properties; temperature, salinity, and volume fraction. To summarise, our model input parameters were: 
- $\sigma_{D C . i c e}-\mathrm{DC}$ conductivity of ice phase

- $S$ - sea ice salinity

- $a / b$ - brine pocket aspect ratio

- $\theta$ - brine pocket orientation from vertical.

These parameters were manually adjusted and set to achieve a rough low temperature agreement between the model output and wheelie bin experimental data. The adjustments were not difficult as each parameter controls a different aspect of the model output: $\sigma_{D C . i c e}$ and $S$ control the magnitude of the conductivity, $a / b$ controls the magnitude of the susceptibilities and $\theta$ controls the anisotropy of the susceptibility and conductivity. The results are shown in Figures 4.25 and 4.26 and the model parameters are summarised in the accompanying tables.

The model input parameters were set to achieve agreement with experimental data in the low temperature limit. In this regime, the magnitudes and anisotropy of the model outputs compare well with the two-electrode experimental data. The largest discrepancy between model and data occurred for the parameter $\epsilon_{\infty}$ - even this was not dramatic. The model is incapable of producing isotropic conductivities and anisotropic susceptibilities as observed in the four-electrode data. The output compares well with experimental $\chi$ and the magnitude of $\sigma_{D C}$. The model input parameters were realistic for both data sets.

The simulated model microstructural evolution between $-5^{\circ} \mathrm{C}$ and $25^{\circ} \mathrm{C}$ produces some of the trends observed experimentally. The bulk experimental parameters exhibit a general decrease with decreasing temperature below $-10^{\circ} \mathrm{C}$. The model demonstrates the same dependency. However, it overestimates the rate of decrease of $\chi$ - particularly the horizontal component. At higher temperatures the four-electrode experimental $\chi$ data increase with decreasing temperature. This trend is perceivably reconcilable with some enforced evolution of brine ellipsoidal dimensions 

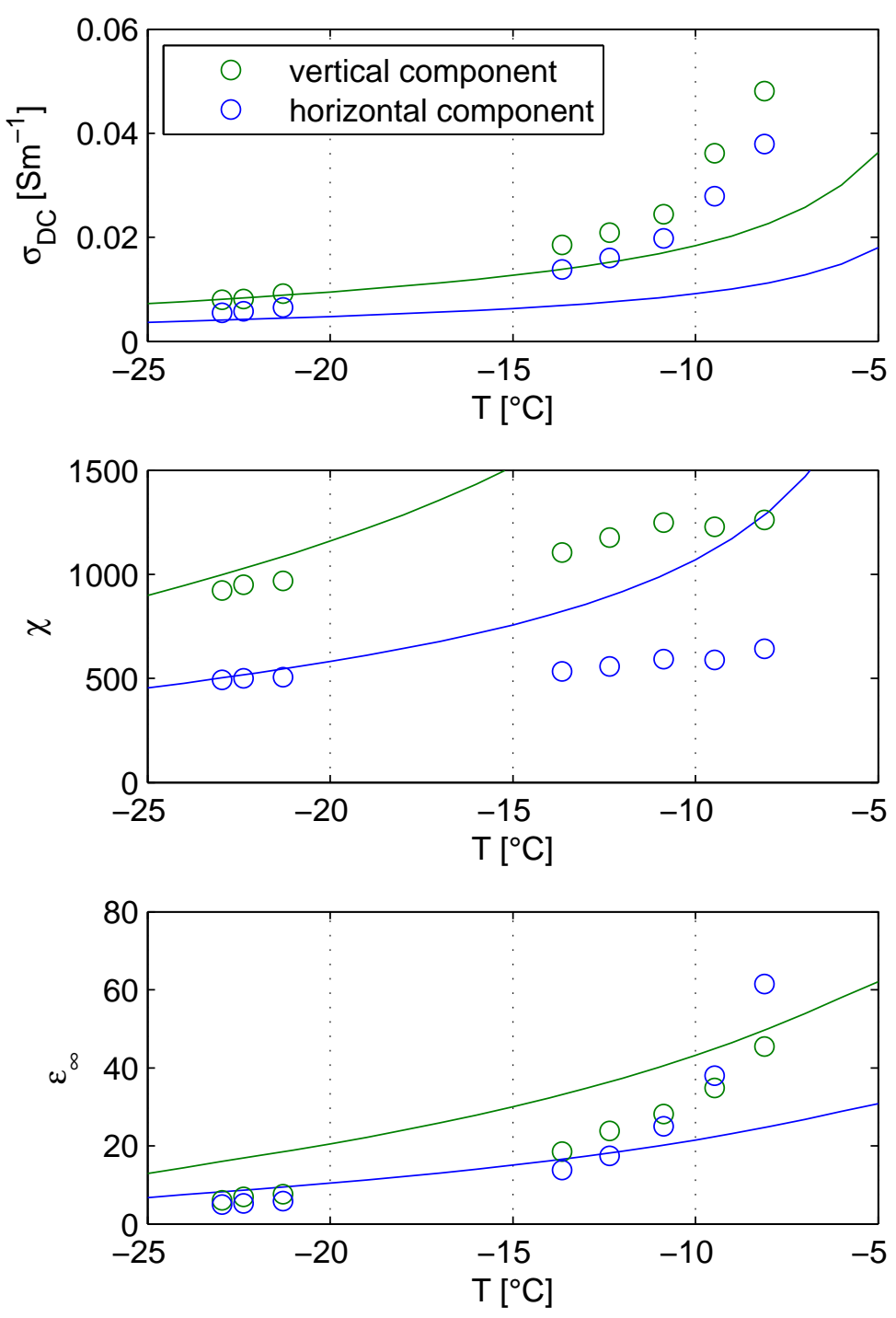

\begin{tabular}{|c|c|c|c|}
\hline$\sigma_{D C . i c e}\left[\mathrm{Sm}^{-1}\right]$ & $\mathrm{S}[\mathrm{ppt}]$ & $\mathrm{a} / \mathrm{b}$ & $\theta$ \\
\hline $0.77 \times 10^{-3}$ & 15 & 32 & $35^{\circ}$ \\
\hline
\end{tabular}

Figure 4.25: Experimental(circles) and model data(solid lines) for horizontal and vertical projections of the real permittivity and conductivity. Experimental data is for the 1-2 two-electrode measurement. 

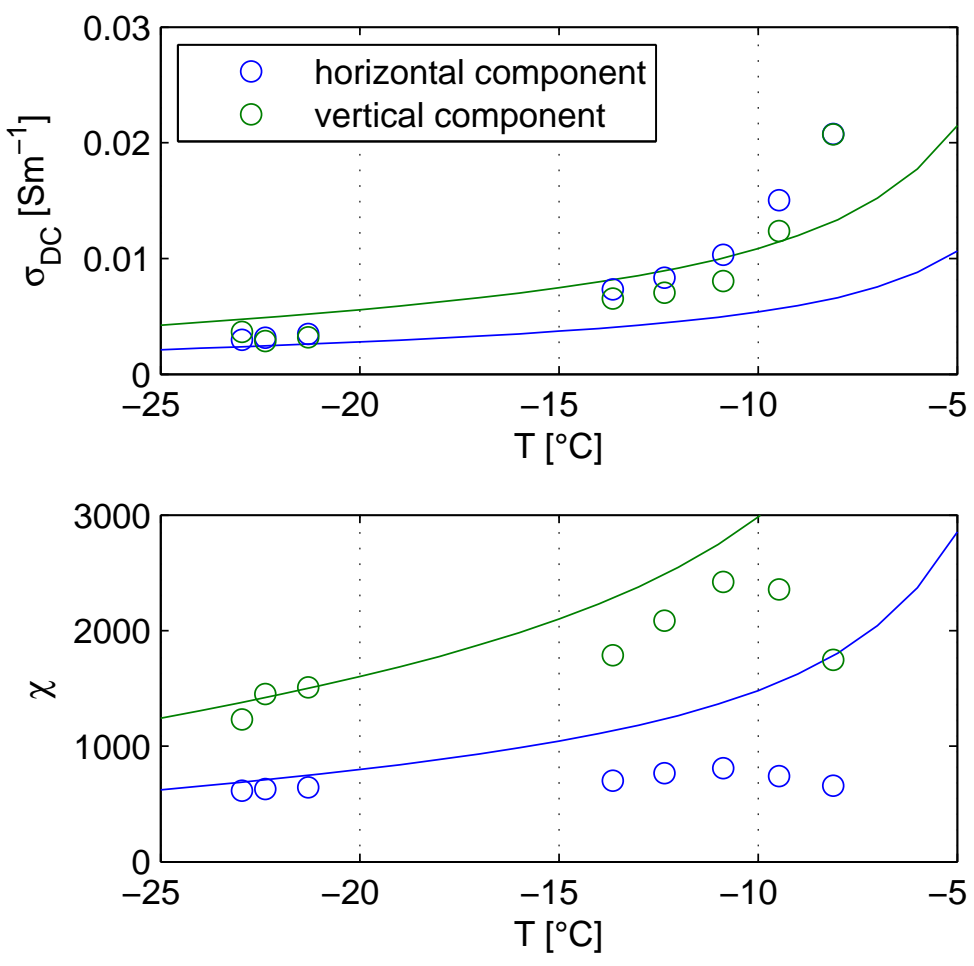

\begin{tabular}{|c|c|c|c|}
\hline$\sigma_{\text {DC.ice }}\left[\mathrm{Sm}^{-1}\right]$ & $\mathrm{S}$ [ppt] & $\mathrm{a} / \mathrm{b}$ & $\theta$ \\
\hline $0.44 \times 10^{-3}$ & 14 & 40 & $35^{\circ}$ \\
\hline
\end{tabular}

Figure 4.26: Experimental(circles) and model data(solid lines) for horizontal and vertical projections of the real permittivity and conductivity. Experimental data is for the four-electrode measurement. 
or a change in sample salinity. However, in this high temperature limit the assumption of dispersed ellipsoids is particularly invalid as brine would exist in unconstrained, interconnecting channels. This is a limitation of the model. The model limitations are:

- The modelled microstructure is a gross simplification of natural sea ice microstructure. It is assumed that all brine exists in ellipsoidal pockets orientated at a constant angle from the vertical, and salinity is constant within the sample. These assumptions would be invalid at high temperatures.

- The sample does not satisfy the idealised requirements of the effective medium approach, as it is small compared to the size of the inclusions and incorporates a relatively small amount of inclusions.

To summarise, utilisation of the 'VTS' model proved that the observed electrical bulk properties of sea ice are compatible with dielectric mixture theory. Realistic model microstructual input parameters produce good agreement with experimental data at low temperatures. This was not achieved at higher temperatures, however some disagreement is hardly surprising considering the limitations of the model. 


\section{Chapter 5}

\section{Summary and Perspective}

The preceding pages represent the vast majority of hours devoted to this project. They outline the thought progression of this MSc, beginning with the grounding provided by the lab freezer experiment. This experiment raised a number of questions and practical issues which the Wheelie bin experiment sought to address. The results and procedures are free standing and applicable beyond the experiments discussed in this thesis. This chapter summarises these results, discusses them in the light of other experimental studies, and considers their relevance and applicability to field sea ice impedance monitoring.

\subsection{The low frequency electrical properties of sea ice}

We performed a series of in-situ impedance measurements on a laboratory grown sea ice sample. Two custom four-electrode measurement cells were constructed for the impedance measurements. They were arranged at horizontal and vertical orientations within the sea ice in order to sample material anisotropy. The measurements were performed across the frequency range $40 \mathrm{~Hz}-1 \mathrm{MHz}$. The measurements monitored the 
development of a columnar microstructure down to a temperature of $-23^{\circ} \mathrm{C}$.

The four-electrode impedance cell was utilised to realise and eliminate the contribution of electrode polarization. We found that it contributed to two-electrode sea ice impedance measurements at frequencies below $20 \mathrm{kHz}$. For a sea ice sample at a temperature of $-8^{\circ} \mathrm{C}$, a sample diameter of $70 \mathrm{~mm}$, and a plate separation of $7 \mathrm{~mm}$, the contribution of electrode polarization was an order of magnitude larger than that of material polarization. For a plate separation of $35 \mathrm{~mm}$, the contribution was three times larger than that of the material. The affect remained a dominating aspect of the two-electrode measurement at a temperature of $-23^{\circ} \mathrm{C}$ - the coldest temperature of the experiment. The four-electrode measurement was insensitive to electrode polarization and measured the true material properties.

The material response was represented in terms of a complex conductivity. This representation offers a number of advantages over the traditional complex permittivity. It was found that the broadband electrical response of sea ice is adequately described by the equation:

$$
\sigma^{*}=\underbrace{\sigma_{D C}}_{1}+\underbrace{K(j \omega)^{m}}_{2}+\underbrace{j \omega \epsilon_{0}\left(\frac{\chi}{1+j \omega \tau}+\epsilon_{\infty}\right)}_{3} .
$$

This model distinguishes bulk and interfacial processes. The three terms represent:

1. bulk material conduction,

2. space charge polarization, and

3. bulk material polarization.

The third term also reveals that the material undergoes a dielectric relaxation. The model was applied to the experimental data and the six parameters were recovered for each measurement using a complex nonlinear fitting procedure. 
Analysis of the bulk material parameters revealed that:

- The relaxation time $\tau$ was temperature dependent and ranged from $2 \times 10^{-6}$ to $8 \times 10^{-6}\left[\mathrm{~s}^{-1}\right]$. The dielectric relaxation was characterised by an activation energy of $E_{a}=0.20 \pm 0.04[\mathrm{eV}]$. This value is compatible with results from low-high temperature experimental studies of $\mathrm{NaCl}$ doped ice.

- The susceptibility $\chi$ of the relaxation was temperature dependant and highly anisotropic. It generally decreased with decreasing temperature and the vertical component was a factor of two times larger than the horizontal. The magnitude ranged from 500-2000 which is much larger than the approximately 100 that it is for ice in isolation.

- The DC conductivity $\sigma_{D C}$ decreased to $0.003\left[\mathrm{Sm}^{-1}\right]$ at low temperatures. It was found to be anisotropic provided that continuous brine channels linked the electrodes.

- The high frequency permittivity $\epsilon_{\infty}$ decreased with temperature and ranged from 60-5. it was weakly anisotropic.

Aspects of the bulk material properties were reconciled with the 'VTS' dielectric mixture model of sea ice. For realistic microstructural inputs and phase properties the model was capable of producing bulk material properties of the magnitude observed experimentally.

The parameter $K$ describes the magnitude of interfacial polarization. It varied between -0.1 and -0.01 for the two-electrode measurements, and between -0.02 and -0.001 for the four-electrode measurements. This demonstrates the dominance of electrode polarization over internal space charge polarization. The parameter $m$ varied between -0.2 and -0.6. Both parameters were anisotropic, however, we were unable to link them with sea ice microstructure. 


\subsection{Perspective}

\subsubsection{Electrode polarization}

The contribution of electrode polarization to a low frequency sea ice impedance measurement can be large. Its dominance depends on both the sample dimensions, and the electrode employed. The affect can be reduced by utilising mesh electrodes which minimise sample contact area. We utilised a four-electrode cell to eliminate it.

Is a four-electrode cell then required for field sea ice impedance measurements? Such an instrument doubles the footprint of the sensor, both in terms of metal in the ice, and connecting measurement cables. It should only be used if absolutely necessary. Electrode polarization only obscures internal space charge polarization. The bulk material properties (described by $\chi, \sigma_{D C}$ and $\epsilon_{\infty}$ ) can be extracted from a twoelectrode measurement using the analytical technique outlined in the previous chapter. We have made steps towards interpreting these bulk material properties in terms of microstructure. A similar link has not been made for the interfacial properties $(K$ and $m)$. If then, there is no practical use for low frequency internal space charge polarization data, then there is little point complicating the measurement with a fourelectrode cell. The parasitic contribution to a two-electrode measurement, and the cells invasiveness, could be reduced by using electrodes made of a finer mesh. Alternatively, and following the lead of Notz et al. (2005), electrode polarization could be exploited as a means of recovering sample salinity.

\subsubsection{Applicability}

Without an impedance analyser capable of high precision measurements across a wide frequency range, this analysis would have been impossible. It is recognised that this technique is unlikely to be applied in full 
outside of the laboratory. Nonetheless, we have realised that different physical processes dominate the electrical response of sea ice across different frequency ranges. It should be possible to perform an impedance measurement at a particular frequency, and be confident that what is measured is due dominantly to either bulk or interfacial processes. Figure 5.1 is presented with this intention - it shows the distinct contributions to the complex conductivity of the bulk and interfacial processes.

The plot reveals that, a measurement performed at $10 \mathrm{kHz}$ measures dominantly bulk material polarization and conduction. If a relaxation time is assumed (the laboratory results presented in the previous chapter could suffice) then the susceptibility can be recovered. Alternatively, if the instrument employed offers some frequency variation, then a Debye fit could be performed on a narrow broadband $(10 \mathrm{k}-100 \mathrm{kHz}$, or even less) impedance measurement to recover the susceptibility and relaxation time. Alternatively, an impedance measurement at $100 \mathrm{~Hz}$ is sensitive to space charge polarization.

\subsubsection{Impedance measurement error}

The least satisfactory aspect of this work was our inability to eliminate the high frequency measurement error. Considerable efforts were made to negate its affect, including:

- using coaxial measurement cables in the appropriate configuration, and

- characterising the cables to allow for post processing open/short/load compensation.

The measurement error affected the reactance most adversely. A positive reactance indicates an erroneous impedance measurement and we assumed that at lower frequencies, where the reactance was negative, the measurement was error free. This assumption confined our accurate 

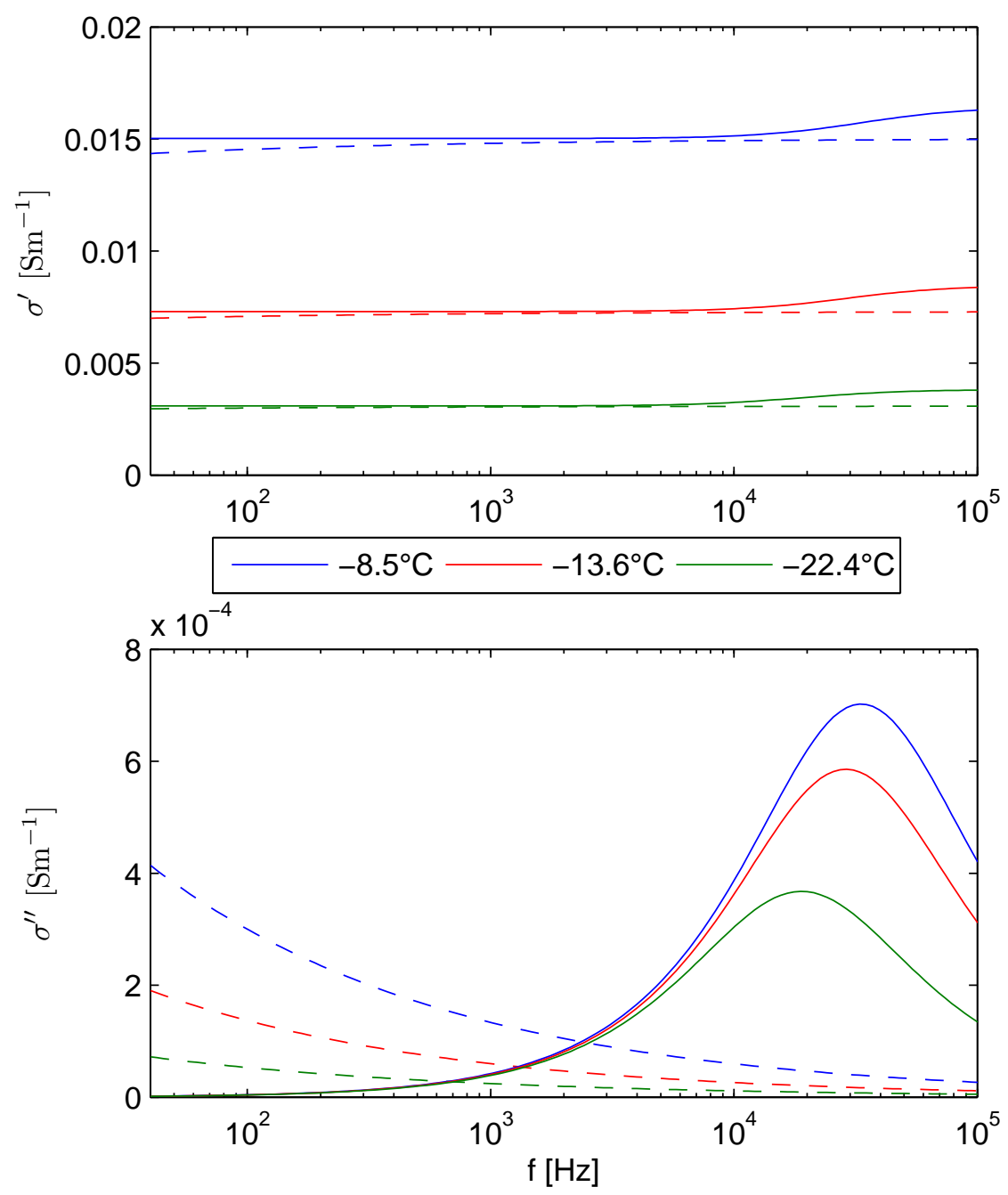

Figure 5.1: The figure shows the distinct contribution of space charge polarization (dashed lines) and bulk polarization (solid lines) to the horizontal component of the complex conductivity of sea ice. $\sigma^{\prime}$ is presented about the level of $\sigma_{D C}$.

impedance measurement range to below $1 \mathrm{MHz}$ for the 1-2 two-electrode measurement, and below $100 \mathrm{kHz}$ for the four-electrode measurement. 
The validity of these limits depends on the frequency dependence of the error - how suddenly it takes affect.

We saw evidence of the impedance measurement error below these limits in the parameter $\epsilon_{\infty}$, as recovered from the four-electrode impedance data. It was in some instances negative and is not presented here. We expect the parameter $\epsilon_{\infty}$ to be the most sensitive to the error. We are uncertain of the errors affect on the recovered $\chi$ and $\tau$.

The measurement error, and the frequency at which it takes hold, depends on the cable length. The horizontal and vertical capacitors have different measurement cable lengths. A cataclysmic undoing of this work would be that the observed relaxation and susceptibility anisotropy were due to different measurement errors caused by different cable lengths. We are confident that this is not the case, and that measurement errors do not significantly affect the results and interpretation presented. This confidence is based on:

- The time constant and activation energy of the relaxation observed match other experimental studies of $\mathrm{NaCl}$ doped ice.

- The susceptibility of the relaxation can be inferred, at frequencies as low $1 \mathrm{kHz}$, from the four-electrode permittivity data shown in Figure 4.12. At low temperatures, the space charge contribution is small, and the real component of the permittivity flattens off at low frequencies. Because $\epsilon_{\infty}$ is small, this level is approximately equal to $\chi$. The real component of the permittivity, read off at these low temperatures and frequencies, is roughly equivalent to the presented values of $\chi$ as produced from fitting the entire measurement frequency range. A frequency of $1 \mathrm{kHz}$ is too low to be affected by measurement cable transmission effects. 


\section{Chapter 6}

\section{Antarctic winter 2009 experiment}

This masters thesis was introduced with an experiment which was to be performed in the McMurdo Sound sea ice over the winter of 2009. This was also the Author's introduction to the project, and it served as the motivation for all subsequent work. The purpose of the laboratory experiments, and all understanding gained from them, was to aid in the interpretation of this field data. Over the course of the year the Antarctic data accumulated, and some minor analysis was performed. However, on the whole, it received little attention compared to the subject matter of the previous three chapters. This prioritisation was in line with the thesis goal. It was always believed that, field data interpretation would be easier after a laboratory grounding.

It turns out that, the advanced analysis performed on the Wheelie bin data can not be applied to the Antarctic data which lacks the accuracy and frequency coverage. Instead, we offer basic geophysical interpretations of the data. The procedure does not follow from the previous chapters and this chapter reads more like an epilogue, than the intended thesis culmination. 


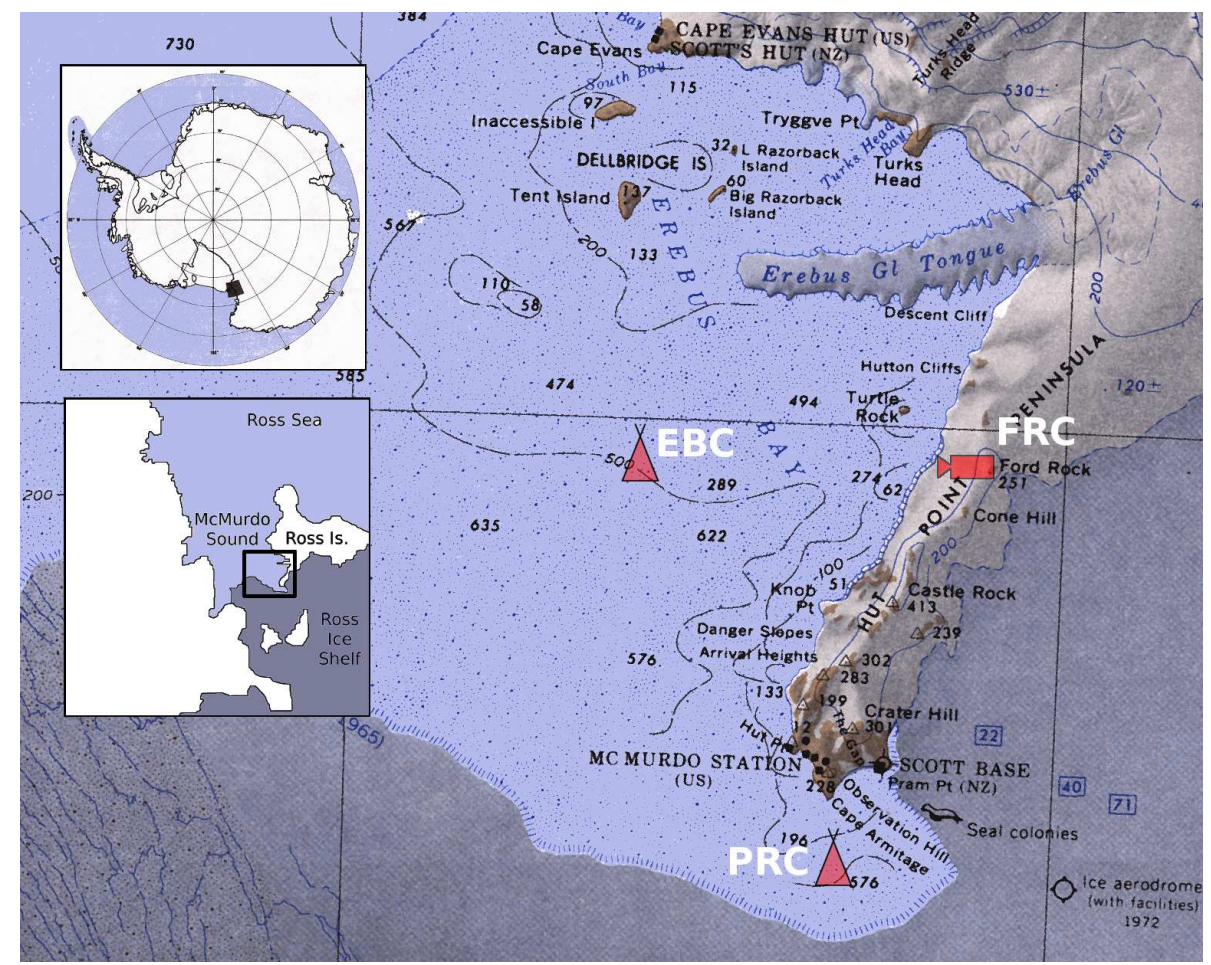

Figure 6.1: Field map showing the position of the mass balance site on the first year sea ice (Erebus Bay Camp - EBC) in McMurdo Sound, Antarctica. Figure reproduced from Gough \& Mahoney, 2009, K131 Winter Sea Ice Growth Processes Monthly Science Report, June 2009.

\subsection{The field site and capacitor array deployment}

The wintering-over team set up Erebus Bay Camp (EBC) on the first year sea ice in Erebus Bay, McMurdo Sound. The exact position of the site is shown on the map titled Figure 6.1. The site was occupied in early July, by which time the ice was $90 \mathrm{~cm}$ thick. Thermistor strings were installed immediately. The temperature profile recorded by the Otago University strings for the winter-autumn period is shown in Figure 6.2. The thermal bottom of the sea ice was estimated, at a particular time, as the depth at which the rate of change of temperature changes abruptly. 


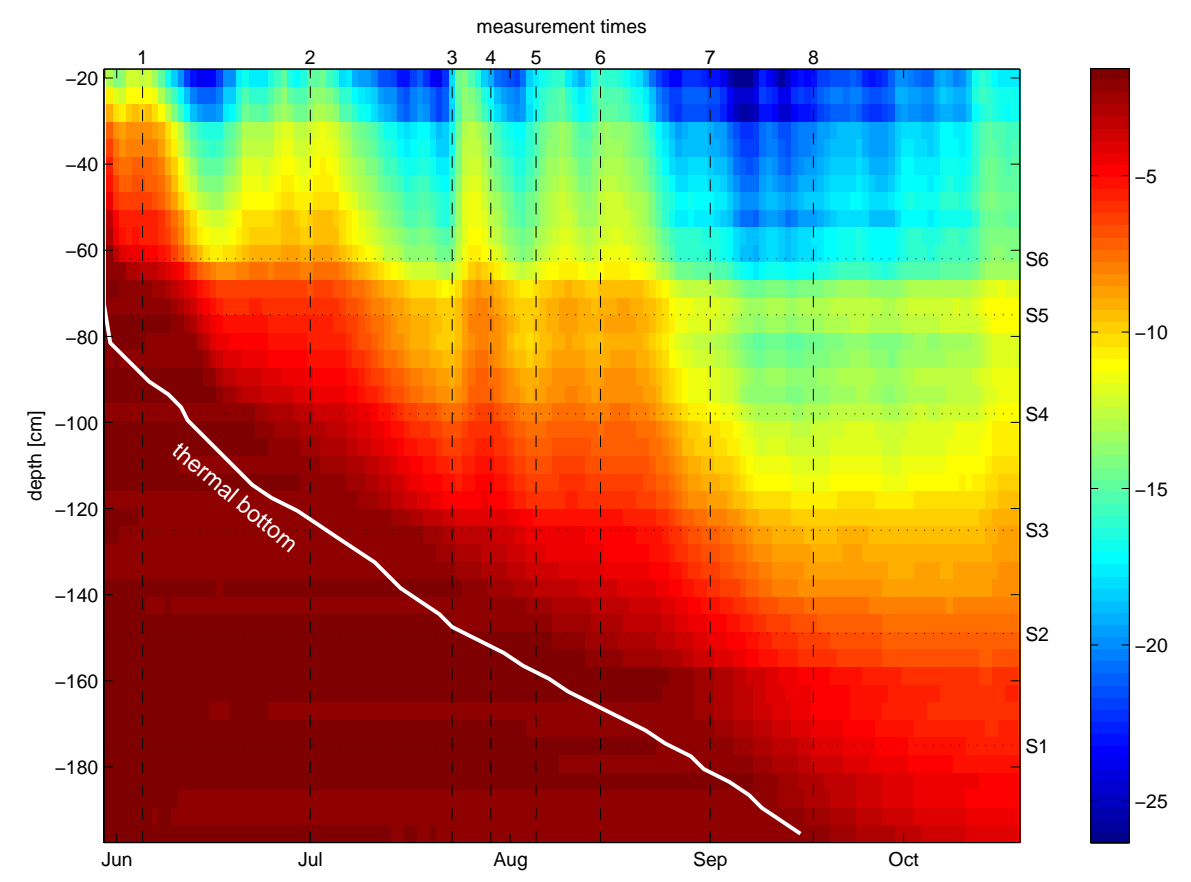

Figure 6.2: Erebus Bay, sea ice temperature profile as measured by the Otago University thermistor string. The colour scale gives the temperature in degrees centigrade. The vertical grid lines mark the measurement occasions and the horizontal grid lines mark the capacitor depths. 


\begin{tabular}{|c|c|c|c|}
\hline capacitor label & depth [cm] & freeze-in date & description \\
\hline S6 & -62 & 05-Jun & vertical, solid plate capacitor \\
\hline S5 & -75 & 05-Jun & horizontal, solid plate capacitor \\
\hline S4 & -98 & 11-Jun & horizontal, mesh plate capacitor \\
\hline S3 & -125 & 03-Jul & horizontal, solid plate capacitor \\
\hline S2 & -149 & 25-Jul & vertical, solid plate capacitor \\
\hline S1 & -175 & 25-Aug & horizontal, solid plate capacitor \\
\hline
\end{tabular}

Table 6.1: Capacitor depths, specifics and estimated freeze-in date. The array was installed on the 5 th of June with all the capacitors deployed in water. S5-S6 refroze immediately.

On the 5th of June a hole was bored through the ice and the capacitor array installed. The borehole was over drilled so that the base of the array sat $49 \mathrm{~cm}$ below the ice surface. This allowed capacitors S1-S4 to extend beyond the sea ice bottom. The borehole refroze around capacitors S6 and S5. The central depth of each capacitor below the sea ice surface is summarised in Table 6.1. The table also shows the estimated freeze-in date of the capacitors, as estimated from the calculated thermal bottom.

\subsection{Impedance measurement data}

Impedance measurements were performed on 10 occasions in total. The timing was slightly sporadic - it depended on when the wintering-over team could make it to the site and our equipment functioning correctly. The team made eight sets of measurements before leaving the continent in October. A contingent from VUW arrived mid November and performed two more sets of measurements. By this time, the sea ice had begun to melt and its thickness was decreasing ${ }^{1}$. The exact measurement timing

\footnotetext{
${ }^{1}$ sea ice temperature data, at the time of these final measurements, was provided by Dykstra, $R$. The ice was approximately $-5^{\circ} \mathrm{C}$.
} 
is summarised in Table 6.2. Note that measurement number 1 was performed immediately after the array was deployed and before the borehole refroze.

\begin{tabular}{|c|c|}
\hline measurement number & date \\
\hline 1 & 05-Jun \\
\hline 2 & 01-Jul \\
\hline 3 & 23-Jul \\
\hline 4 & 29-Jul \\
\hline 5 & 05-Aug \\
\hline 6 & 15-Aug \\
\hline 7 & 01-Sep \\
\hline 8 & 17-Sep \\
\hline 9 & 17-Nov \\
\hline 10 & 20-Nov \\
\hline
\end{tabular}

Table 6.2: Impedance measurement occasions.

Impedance measurements were performed using the custom impedance analyser described by Gouws et al. (2009). The measurements were made across the frequency range of $10 \mathrm{kHz}-100 \mathrm{kHz}$. However, this coverage was often incomplete due to equipment failures and incorrect instrument settings. A complete data series was obtained in just a few narrow frequency bands.

A complete impedance sweep, made in late November, is shown in Figure 6.3. The form of this data was typical of the Antarctic measurements. We realised that the custom built impedance analyser was inaccurate across some of this frequency range. The evidence of this inaccuracy was:

- At low frequencies, the real component of the measured impedance increases steeply with frequency. This is in contrast to all laboratory measurement in which the resistance decreases gently with frequency. This same dependence occurred when the capacitors 

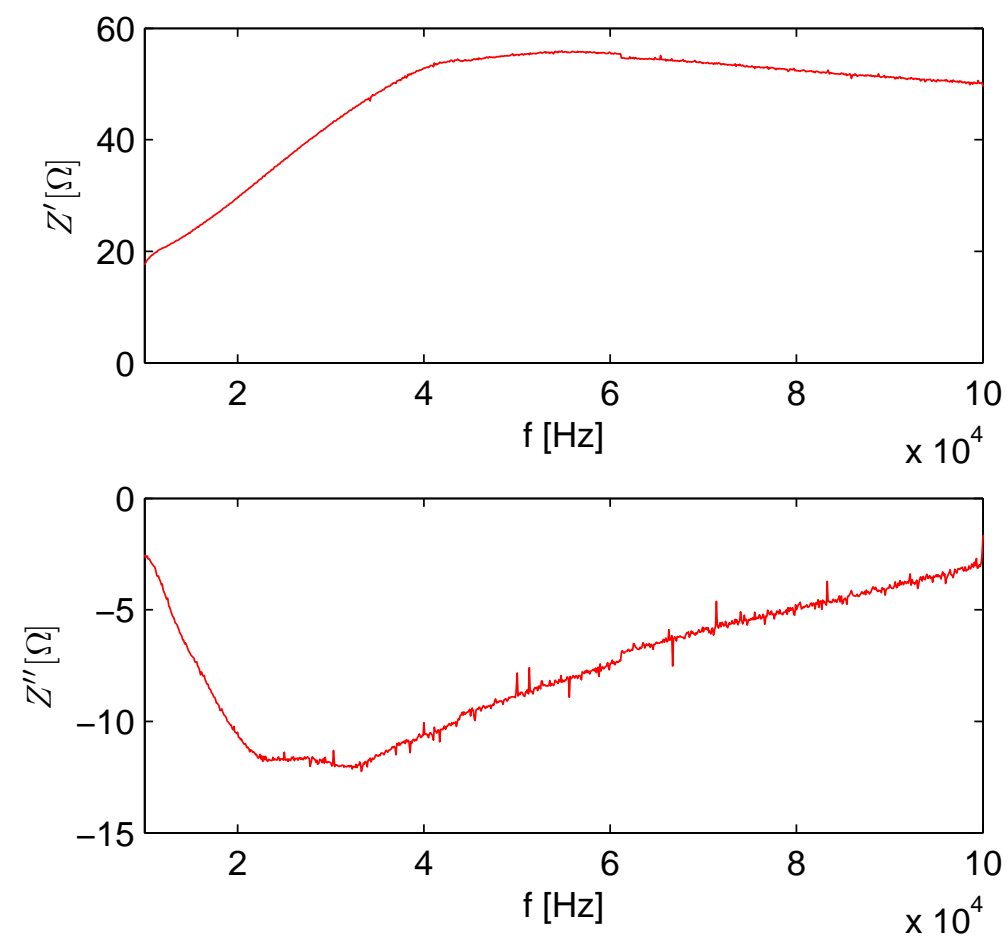

Figure 6.3: Impedance measurement 10 across $\mathrm{S} 3$ for an ice temperature of $-7.2^{\circ} \mathrm{C}$ - typical impedance data from the Antarctic experiment.

were still deployed in sea water, which suggests that it is not a physical result produced by some novel sea ice type.

- At low frequencies, the measured resistance of sea water was negative. This is entirely unphysical.

At higher frequencies $(>50 \mathrm{kHz})$ the resistance flattens off, and then decreases with increasing frequency. It looks more like the laboratory data and we have some confidence in its accuracy.

The impedance measurement inaccuracy, and limited frequency range, meant that the advanced analysis performed on the wheelie bin data could not be applied here. Instead, we concentrated on the impedance measurements at a frequency of $35 \mathrm{kHz}$. This is the highest frequency 
at which we have a nearly continuous measurement record ( 9 of the 10 impedance measurements covered this frequency and the low frequency impedance for 01-Sept was extrapolated to this frequency). Figure 6.4 shows the temporal variation of the $35 \mathrm{kHz}$ impedance.
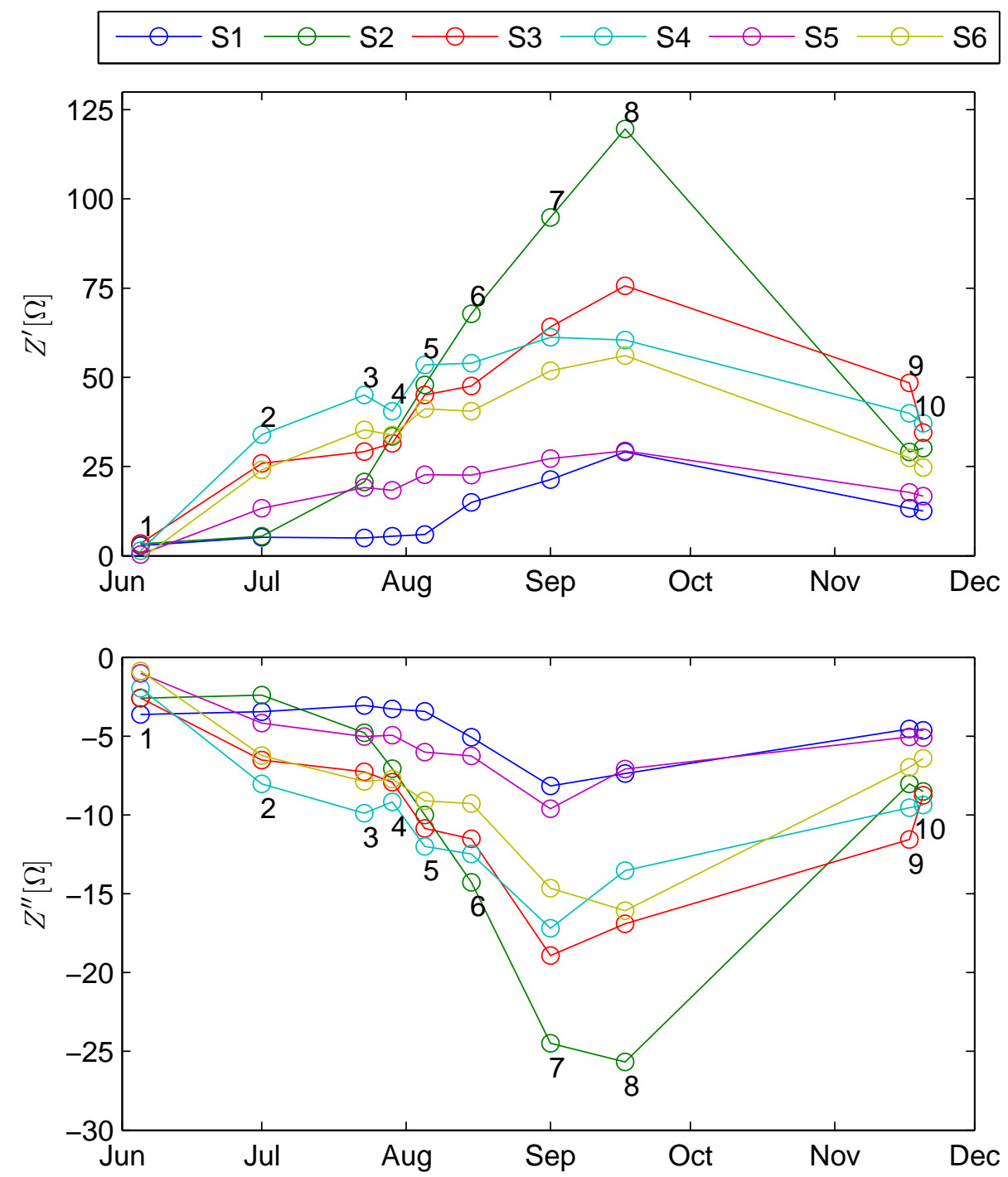

Figure 6.4: The real and imaginary impedance components at $35 \mathrm{kHz}$ for each of the capacitors. The numbers denote the measurement occasions. 


\subsection{Interpretation}

The interpretation was complicated because of the number of variables the impedance depends on. These are:

- measurement frequency,

- sea ice temperature,

- salinity, and

- microstructure geometry.

The frequency dependence was negated by analysing only $35 \mathrm{kHz}$ impedance data. The temperature of the sea ice around the capacitors was estimated from thermistor string data. We ignored the microstructural dependence and assumed that the measured resistance of sea ice, at a given temperature, depended only on salinity. It was then possible to infer sea ice salinity changes from variation in the resistance, coupled with measured temperature changes. Figures 6.5 and 6.6 plot the measured sensor resistance vs sea ice temperature at an equivalent depth.

The plots include resistance and temperature data from the lab freezer experiment $^{2}$ at $35 \mathrm{kHz}$. In the lab freezer chapter, it was acknowledged that these represent unnaturally high salinity sea ice. This was because the finite container allowed nowhere for the brine to drain. The vertically orientated capacitor ('Lab freezer 2' on figures) extended into a pool of brine at the top of the container and measured brine saturated sea ice. The horizontal capacitor ('Lab freezer 1' on figures) enclosed unnaturally high salinity sea ice. These measurements were useful bounds for interpreting the Antarctic data as they represented, roughly, samples of constant salinity. As displayed in Figures 6.5 and 6.6, they are lines of constant salinity or 'isohalines'. Any change in the temperature/resistance state

\footnotetext{
${ }^{2}$ no data is included from the wheelie bin experiment because the measurement cells were different.
} 


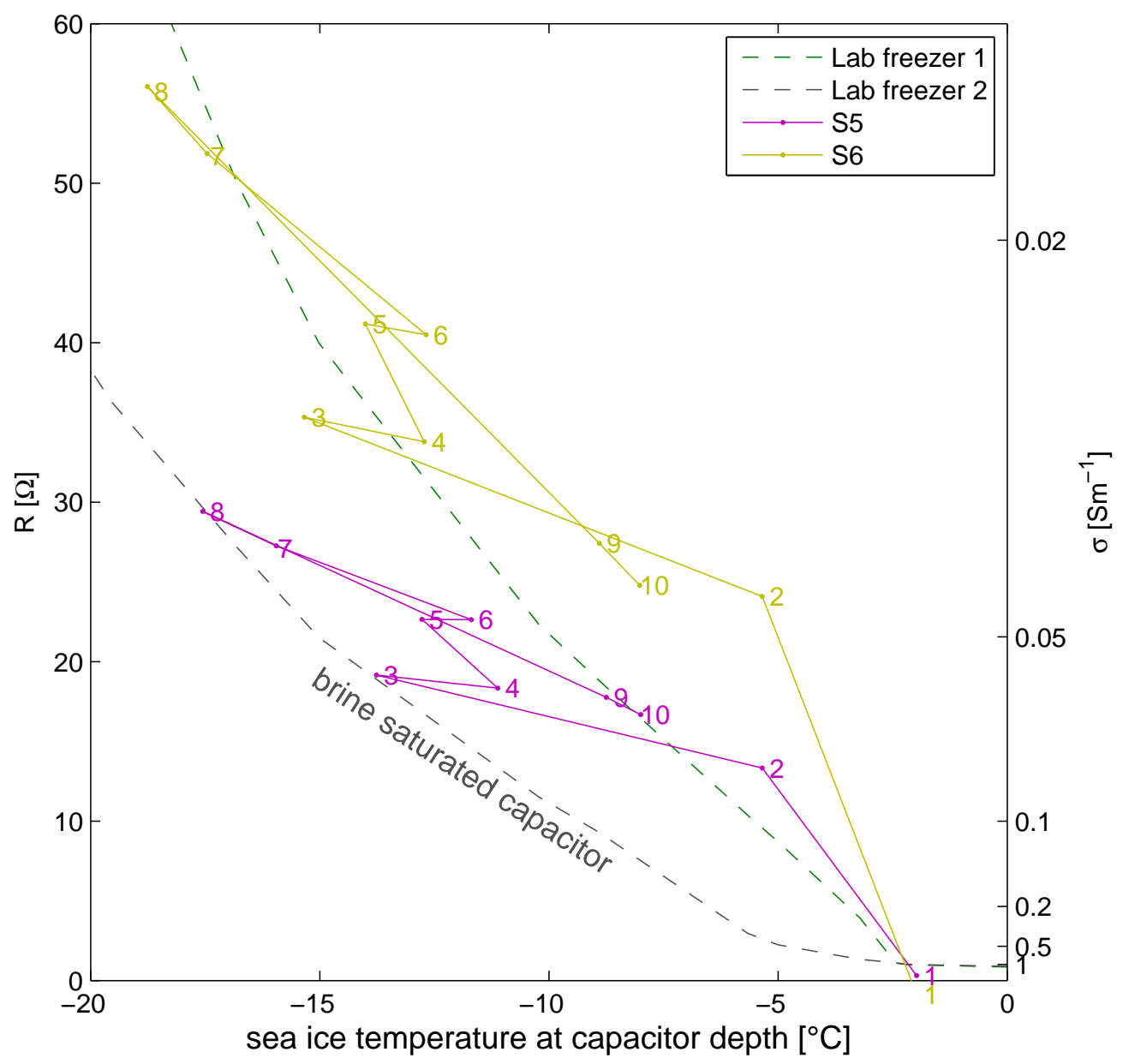

Figure 6.5: Temperature resistance plot for capacitors S5 and S6 around which the borehole re-froze. 
of a cell, which did not follow a similar isohaline, indicates a change of salinity in the cell. If the state moves towards the lab freezer isohalines, then the Antarctic sea ice salinity has increased. If the state moves away from the isohalines, then the salinity has decreased. If the state follows a curve which moves neither towards or away from the isohalines, then the salinity was assumed constant. Only large deviations were inferred as evidence of salinity changes.

Capacitors S5 and S6, which were enclosed in a refrozen bore hole, showed higher salinities than the other capacitors. Sea ice formed in this manner would be expected to have a higher salinity, as the natural brine drainage process does not occur. The two capacitors showed little variation in salinity throughout the course of the experiment and S5 measured higher salinity sea ice than S6. This is likely due to its horizontal orientation which further inhibits brine drainage. Both capacitors showed a salinity increase between measurements 2-3 and S5 showed brine drainage between measurements 8-9.

Oceanographic surveys conducted at EBC indicated that there was considerable interaction between the sea ice and the underlying supercooled ocean. The sea water was supercooled by the surrounding ice sheets. Frazil ice was observed in the water column and a platelet ice structure formed at depths below $1.25 \mathrm{~m}$. A 'platelet' ice structure describes randomly orientated brine pockets - as opposed to the typical columnar structure. The impedance measurements seem to indicate that, the capacitor array provided an accumulation point for this frazil ice. At the time of deployment, capacitors S1-S4 extended into the unfrozen sea water below the sea ice. Subsequent impedance measurements demonstrate that capacitors S1-S3's resistance increased before sea ice has formed at an equivalent depth. This process is indicated by the box in the lower right hand corner of Figure 6.6.

Out of all the capacitors, S2 suggested the lowest salinity sea ice. This can potentially be reconciled with its vertical orientation which 


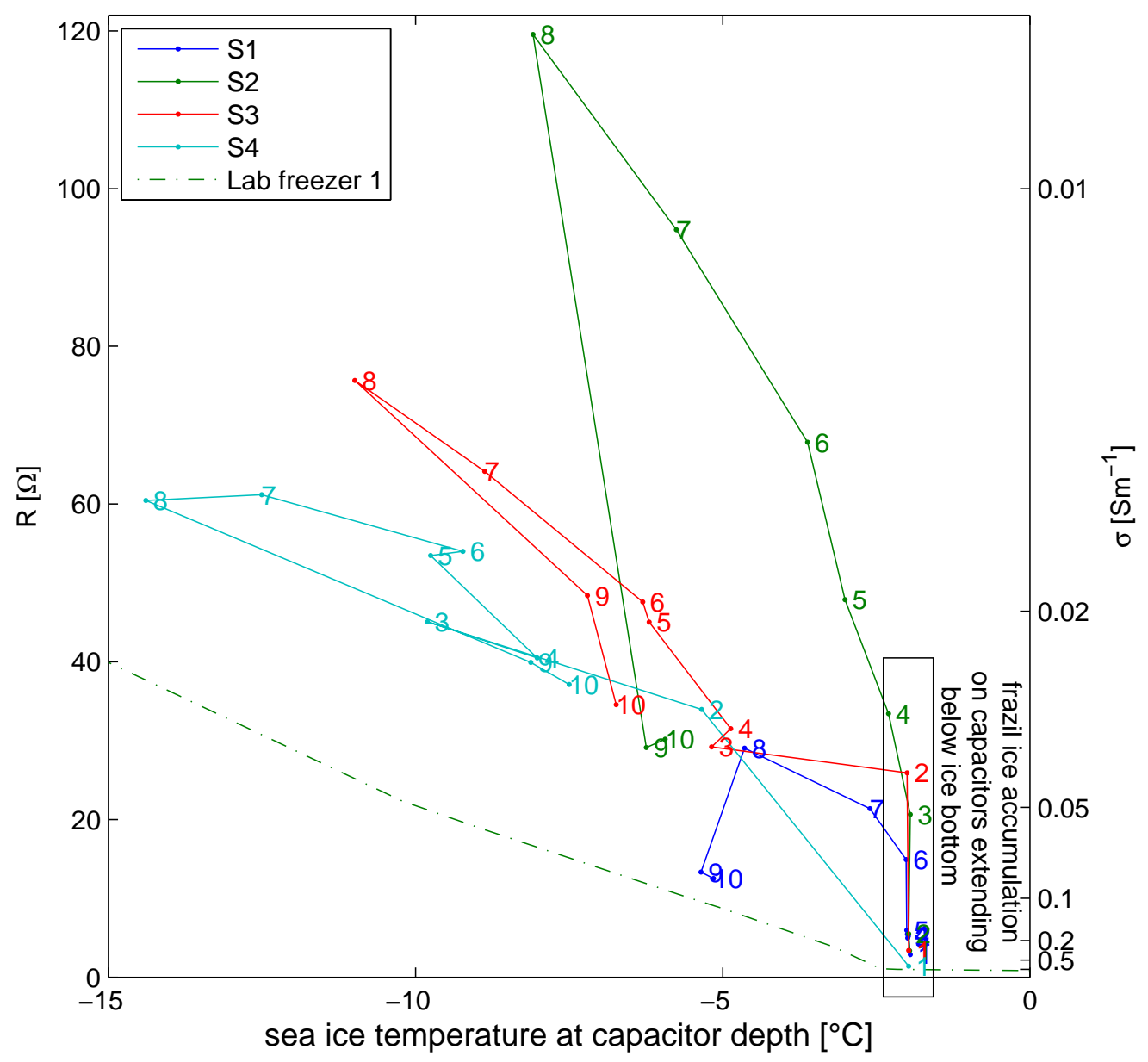

Figure 6.6: Temperature resistance plot for capacitors S1-S4 around which sea ice formed. 
would have offered uninhibited brine drainage during the early stages of sea ice envelopment. It may be the only capacitor which does not impinge on natural sea ice formation. Alternatively, if we drop the assumption that the measured resistance is independent of brine pocket geometry, then the large measured resistances could be due to a columnar microstructure - which was not observed at this depth in extracted ice cores. Over the two month period between measurements 8 and 9 the salinity increased dramatically and the capacitor ended up in a similar temperature/resistance state to the other capacitors.

Capacitor S4 is made of mesh which should facilitate brine drainage. It measured a constant salinity throughout all 10 impedance measurements which was larger than S2. S3 measured a similar salinity to S4 and showed dramatic brine drainage over a short 3 day interval (between measurements 9-10). S1 froze-in last and has a similar salinity to S4 during ice growth. The salinity increased during the melt.

\subsection{Future work}

The prospect of automated sea ice microstructure monitoring means that, in the future, similar experiments can be expected at both ends of the Earth. VUW have been contracted by the University of Alaska, Fairbanks (UAF) to produce a similar device for deployment in the landfast, first year sea ice in Barrow, Alaska. VUW is also considering preparing a device for another Antarctic campaign.

The dielectric array deployed, and impedance instrument utilised, in the Antarctic experiment were prototypes. Like most prototypes, aspects of their design proved unsuitable for the requirements of the job and need revising. The most noticeable inadequacy was the inaccuracy of the impedance analyser. An obvious priority for the VUW group is to develop an instrument capable of accurate sea ice impedance measurements across some frequency range. Realistically, the frequency range will be low 
$(<100 \mathrm{kHz})$ to avoid the contribution of measurement cable residuals. The instrument should undergo extensive testing in a laboratory environment before being sent to the field.

The next generation capacitor array should use mesh electrodes exclusively. The wheelie bin experiment, performed by the Author, demonstrated the capability of such capacitors. There is no justification for embedding large sections of metal within the sea ice.

One result from the Antarctic experiment, regarding the practical capability of these devices, is concerning. This was the function of the unfrozen capacitors as accumulation points for frazil ice. The fundamental assumption of the experiment was that the structure did not impinge on natural sea ice formation. If it does significantly then, the microstructure of the measured sea ice could differ significantly from that of the surrounding natural sea ice, and a microstructural interpretation of the measurements is worthless. This may be less of an issue at other field sites where the ice growth is columnar. The impingement of the structure on natural sea ice formation requires further investigation.

\subsection{Conclusion}

The Antarctic experiment was an unprecedented attempt to monitor sea ice microstructure in-situ, throughout a long period of the annual cycle. Instrument limitations restricted what could realistically be done with the impedance data. We analysed the real component of the $35 \mathrm{kHz}$ impedance data. We considered the resistance/temperature state of the cell to be representative of sea ice salinity and inferred changes in this state in terms of salinity changes. This interpretation was qualitative. The most important interpretations were:

- the salinity of the sea ice in the refrozen borehole was unnaturally high, 
- the capacitors served as accumulation points for frazil ice in the water column,

- during ice growth, capacitor S2 measured a much smaller salinity than the other capacitor. This may be due to its vertical orientation which did not inhibit brine drainage,

- the salinity of capacitor S2 increased dramatically during the melt, and

- the salinity of capacitor S3 decreased dramatically over a 3 day period.

The experiment proved the capability of sea ice impedance monitoring to track changes in sea ice microstructure and it was a necessary first step in an ambitious, long term project. 


\section{Appendix A}

\section{Impedance compensation}

Open/short/load compensation is a technique used to correct for impedance measurement errors. It represents the residuals and phase error of test leads as a linear, 4-terminal network. The relationship between the current and voltage, at the input $\left(I_{1} \& V_{1}\right)$ and output $\left(I_{2} \& V_{2}\right)$ terminals is given generally by:

$$
\begin{gathered}
V_{1}=A V_{2}+B I_{2} \\
I_{1}=C V_{2}+D I_{2} .
\end{gathered}
$$

The parameters A,B,C,D fully characterise the network and are determined by impedance measurements made with the following loads:

- a short circuit load,

- an open circuit load,

- a standard reference load.

The measured impedance can then be transformed into the true impedance using the formula

$$
Z_{\text {cell }}=\frac{\left(Z_{S C}-Z_{m}\right)\left(Z_{S M}-Z_{O C}\right)}{\left(Z_{m}-Z_{O C}\right)\left(Z_{S C}-Z_{S M}\right)} Z_{S T D}
$$

Note that these impedances are complex. Their definitions are: 


\begin{tabular}{|c|l|}
\hline $\mathrm{Z}_{\text {cell }}$ & true cell impedance \\
\hline $\mathrm{Z}_{m}$ & measured impedance of the cell and cables \\
\hline $\mathrm{Z}_{S C}$ & measured impedance with a short circuit load \\
\hline $\mathrm{Z}_{O C}$ & measured impedance with an open circuit impedance \\
\hline $\mathrm{Z}_{S M}$ & measured impedance of standard load \\
\hline $\mathrm{Z}_{S T D}$ & true impedance of standard load \\
\hline
\end{tabular}

A less accurate open/short compensation can be performed without a reference load. The correction is performed using the equation:

$$
Z_{\text {cell }}=\frac{Z_{S C}-Z_{m}}{Z_{m}-Z_{O C}} Z_{O C}
$$


Appendix B

Additional wheelie bin data 

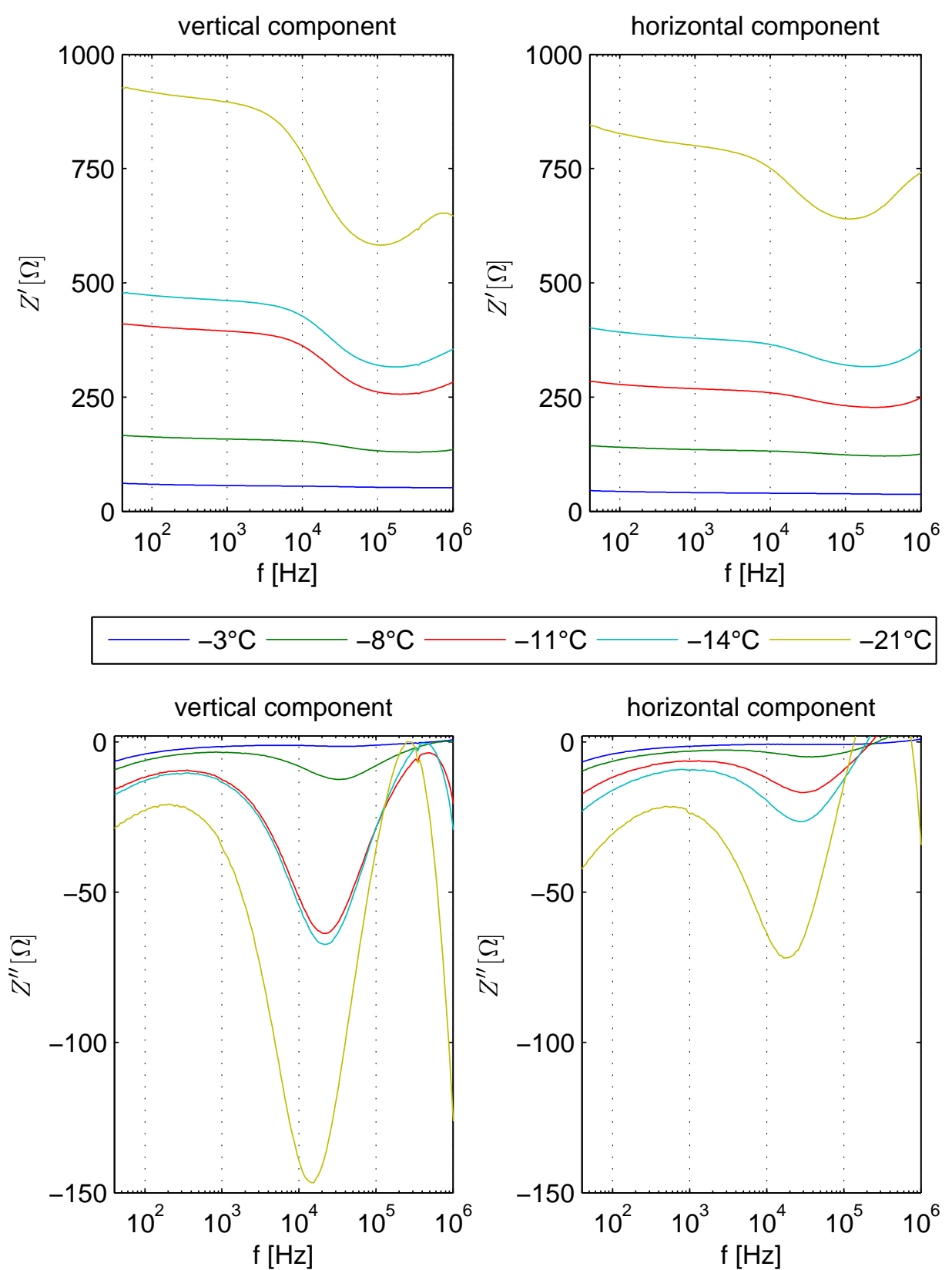

$-11^{\circ} \mathrm{C}--14^{\circ} \mathrm{C}-21^{\circ} \mathrm{C}$

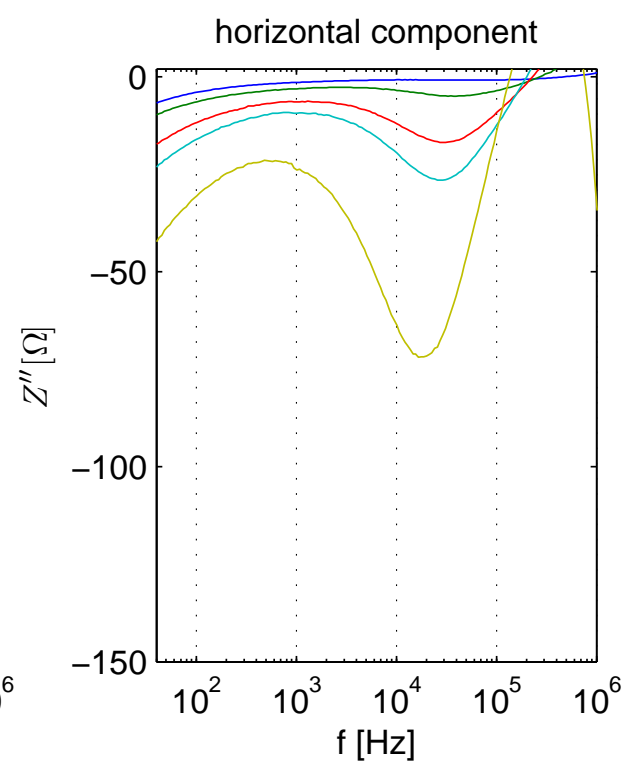

Figure B.1: Vertical and horizontal components of sea ice impedance for a two-electrode measurement across electrode pair 2-3. 

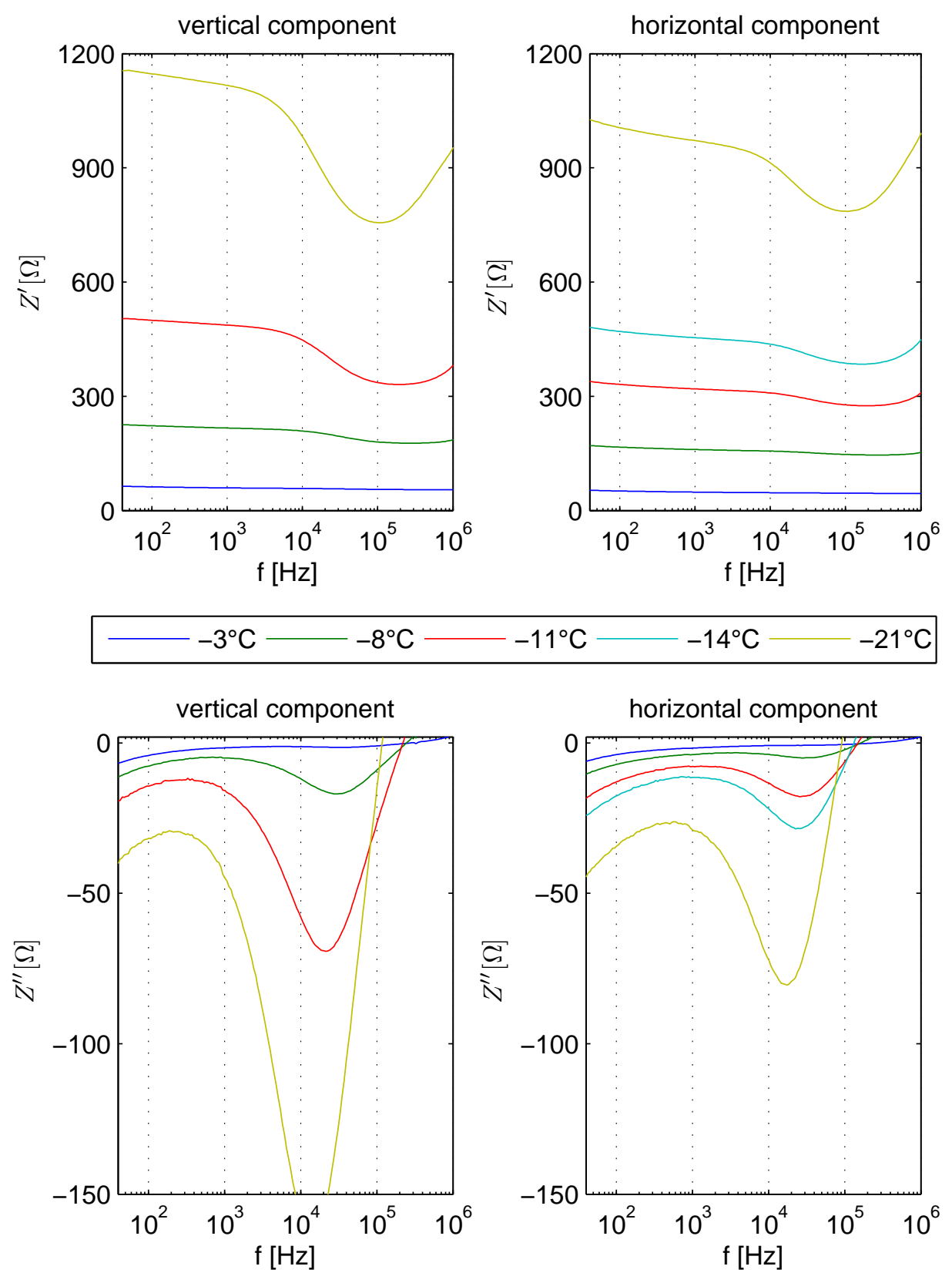

$-11^{\circ} \mathrm{C}--14^{\circ} \mathrm{C}--21^{\circ} \mathrm{C}$

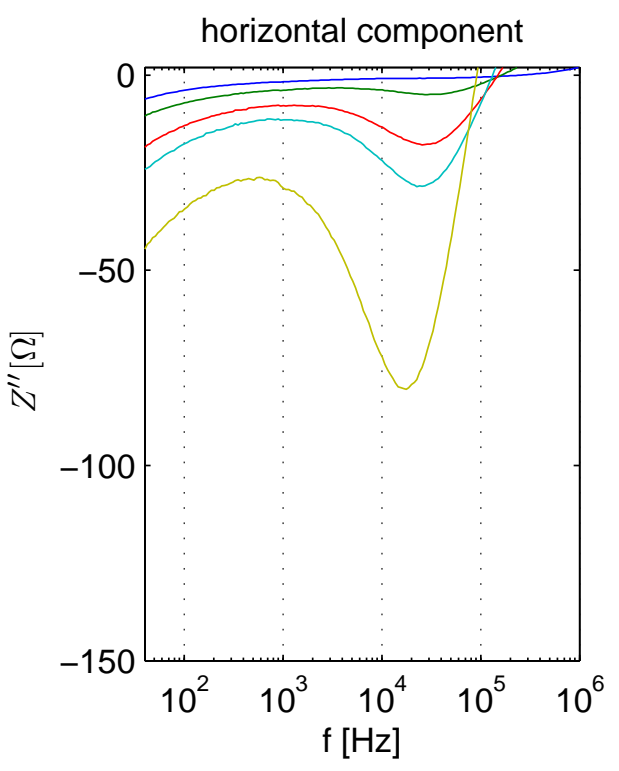

Figure B.2: Vertical and horizontal components of sea ice impedance for a two-electrode measurement across electrode pair 1-4. 

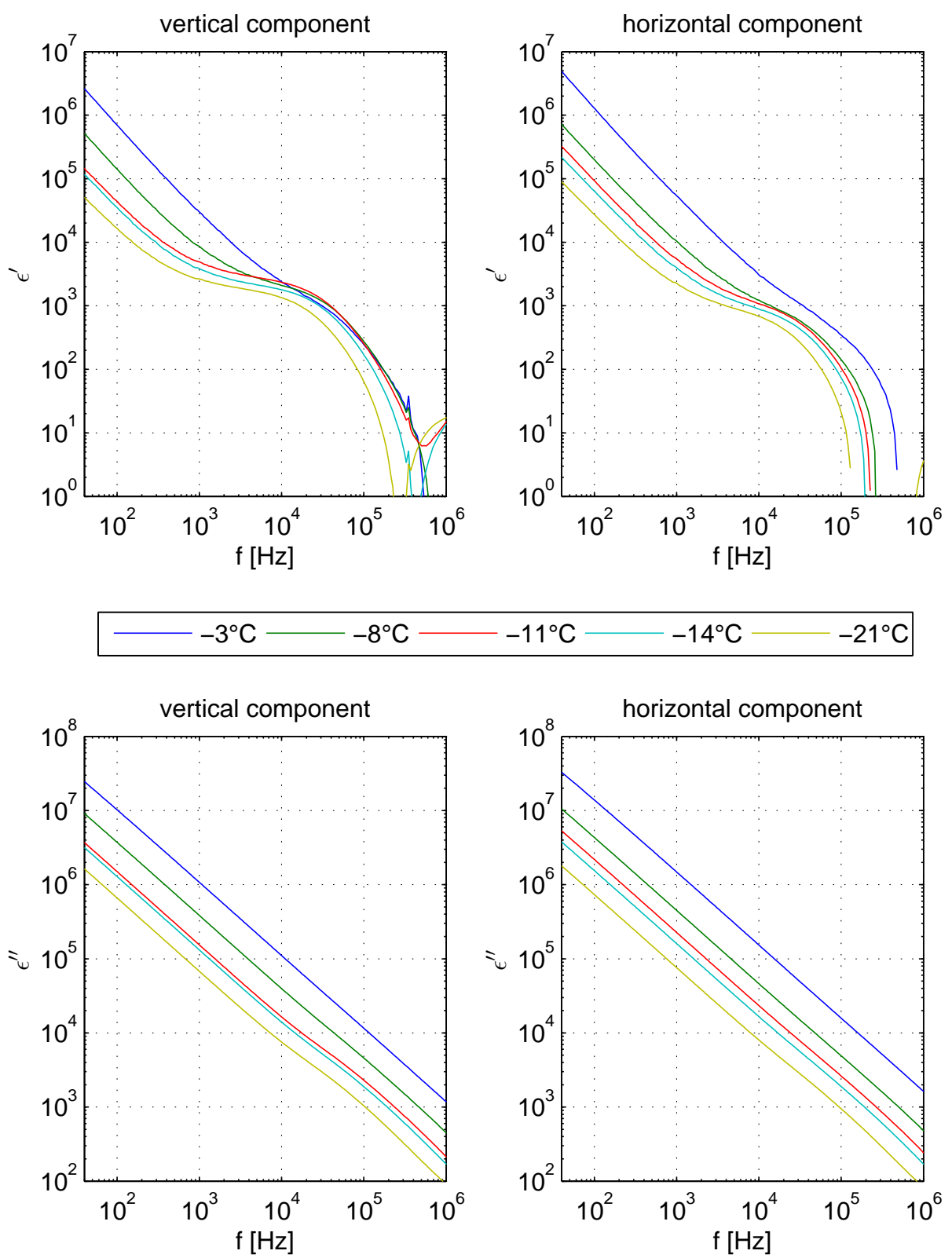

Figure B.3: Vertical and horizontal components of sea ice permittivity for a two-electrode measurement across electrode pair 2-3. 

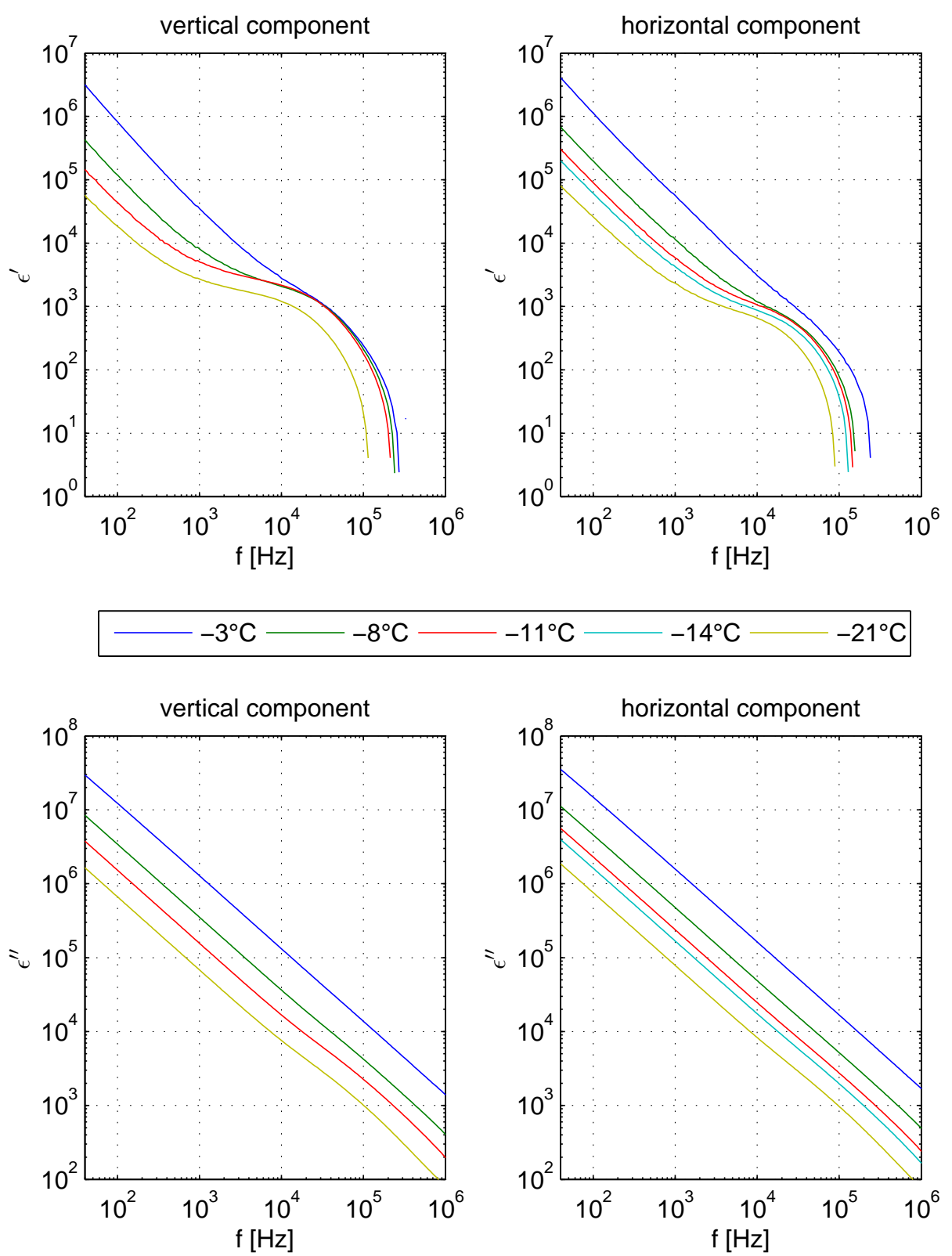

Figure B.4: Vertical and horizontal components of sea ice permittivity for a two-electrode measurement across electrode pair 1-4. 

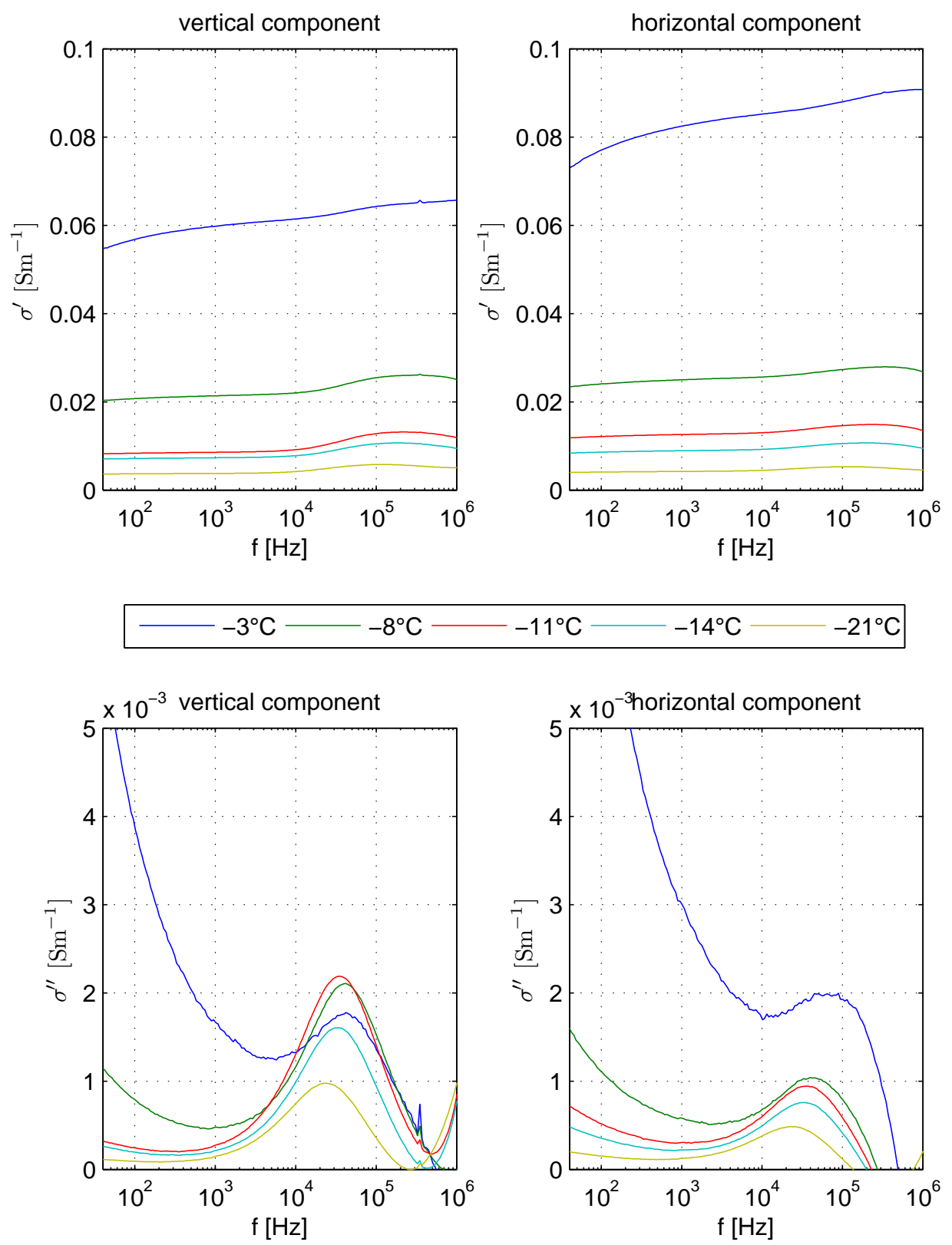

Figure B.5: Complex conductivity representation of the horizontal component of sea ice response for a two-electrode measurement across the electrode pair 2-3. 

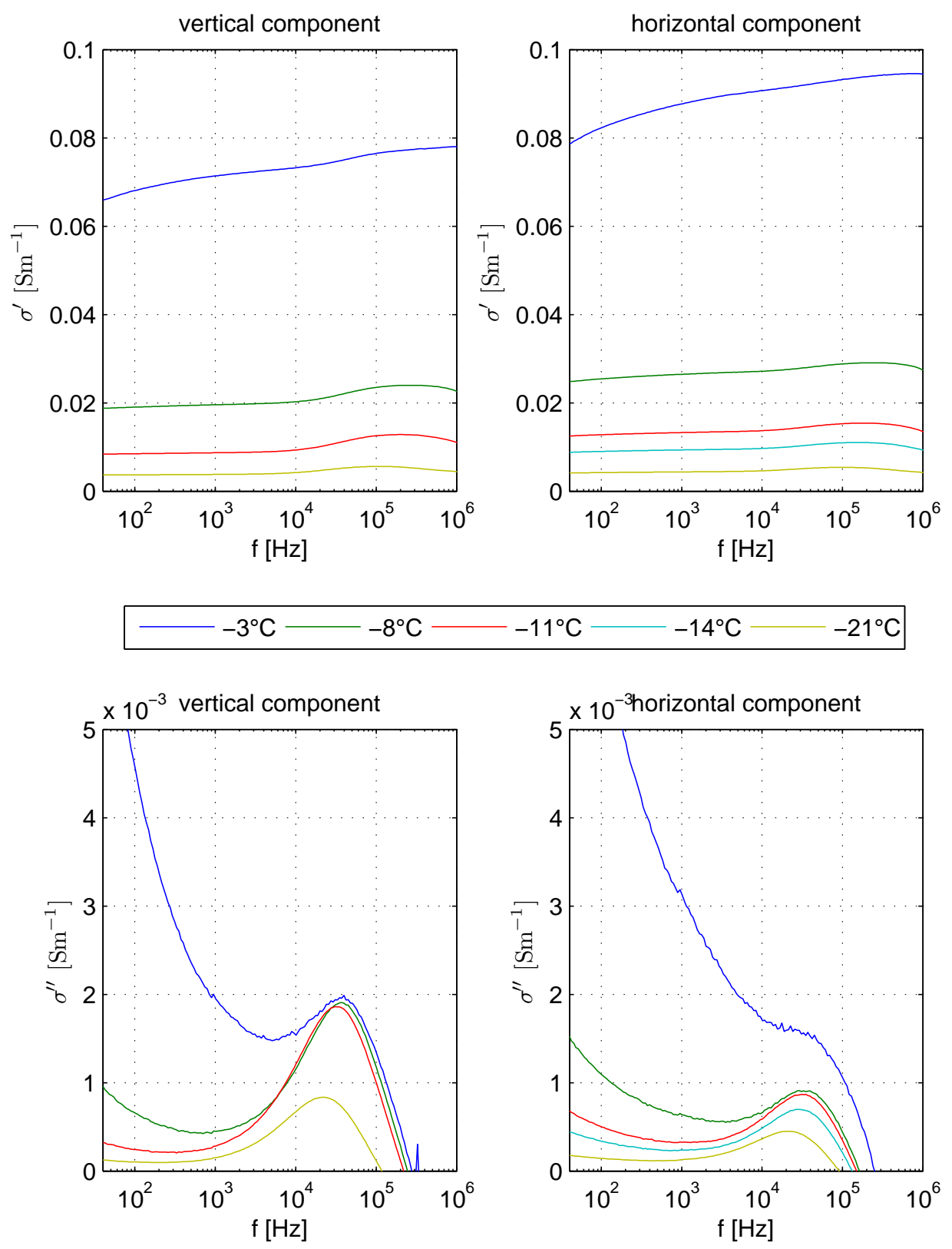

Figure B.6: Complex conductivity representation of the horizontal component of sea ice response for a two-electrode measurement across the electrode pair 1-4. 


\section{Appendix C}

\section{A Debye relaxation in a lossy material}

A Debye relaxation is described by the equation:

$$
\epsilon^{*}=\epsilon_{\infty}+\frac{\chi}{1+j \omega \tau}
$$

where $\chi$ is the susceptibility of the low frequency polarization mechanism, $\epsilon_{\infty}$ is the high frequency permittivity and $\tau$ is the relaxation time constant. The expression traces out a semicircle in the complex permittivity plane as shown in Figure C.1. The data series were generated using parameters $\chi=1, \tau=1 \times 10^{-5}\left[\mathrm{~s}^{-1}\right]$ and $\epsilon_{\infty}$ between $0-0.1$.

The complex permittivity equation can be modified to include the contribution of conductive loss. It becomes:

$$
\epsilon^{*}=\epsilon_{\infty}+\frac{\chi}{1+j \omega \tau}-j \frac{\sigma_{D C}}{\epsilon_{0} \omega}
$$

Figure C.2 shows $\epsilon^{*}$ for a medium where DC conduction dominates dielectric loss. The equation parameters were $\chi=1, \tau=1 \times 10^{-5}\left[\mathrm{~s}^{-1}\right]$ , $\epsilon_{\infty}$ between 0-0.1 and $\sigma_{D C}=2 \times 10^{-6}\left[\mathrm{Sm}^{-1}\right]$. 

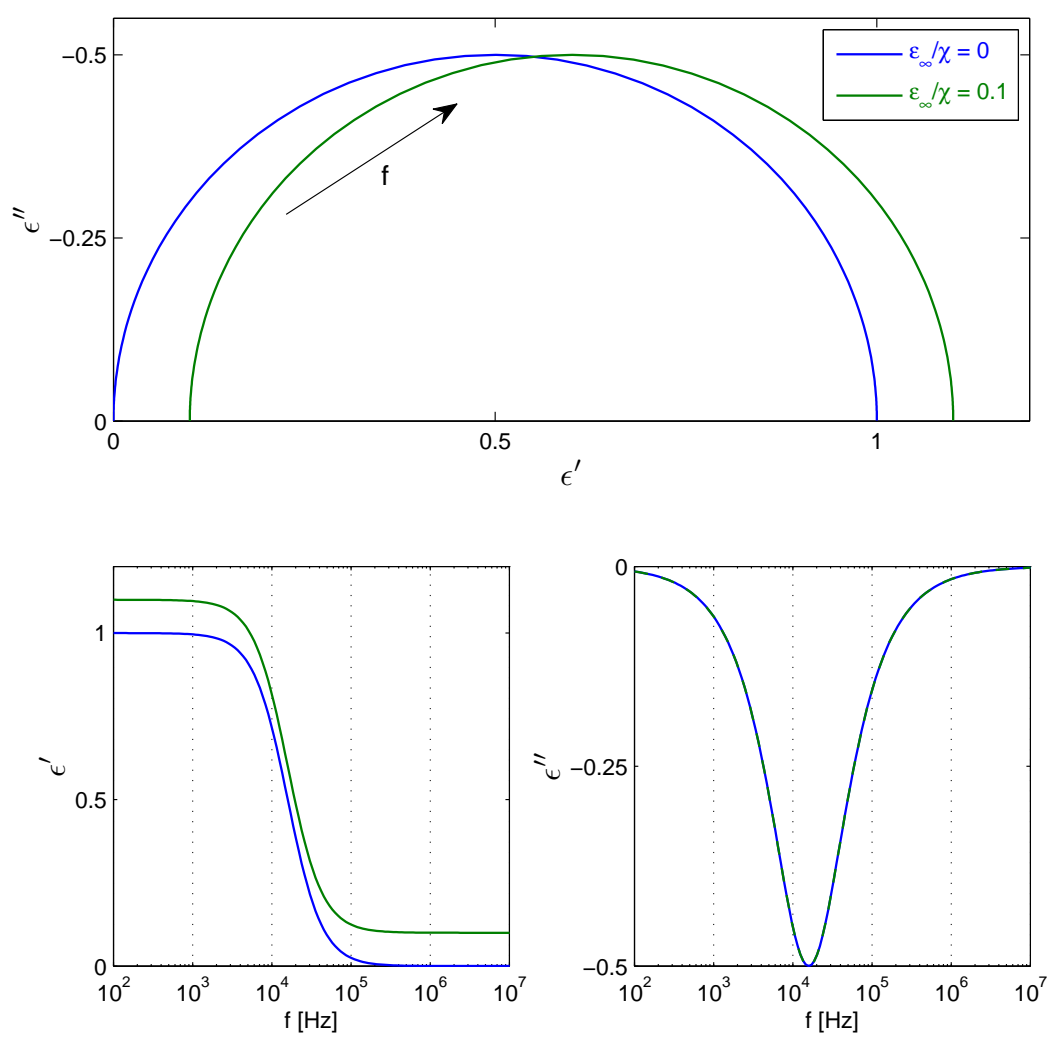

Figure C.1: Complex permittivity representation of a Debye relaxation.

Representing the data in terms of a complex permittivity is not particularly useful. If the DC conduction term was zero then the data would trace out a semicircle in the complex plane. Instead, the imaginary component of the permittivity spans seven decades ( $1 / \omega$ dependence) and shows almost no sign of dielectric loss. Evidence of the relaxation process is only clear in $\epsilon^{\prime}$. If other dispersions occur on this time scale then the experimenter could completely miss identifying the relaxation!

A complex conductivity representation is a superior way to visualise and identify Debye like relaxations in a conductive medium. A conduc- 

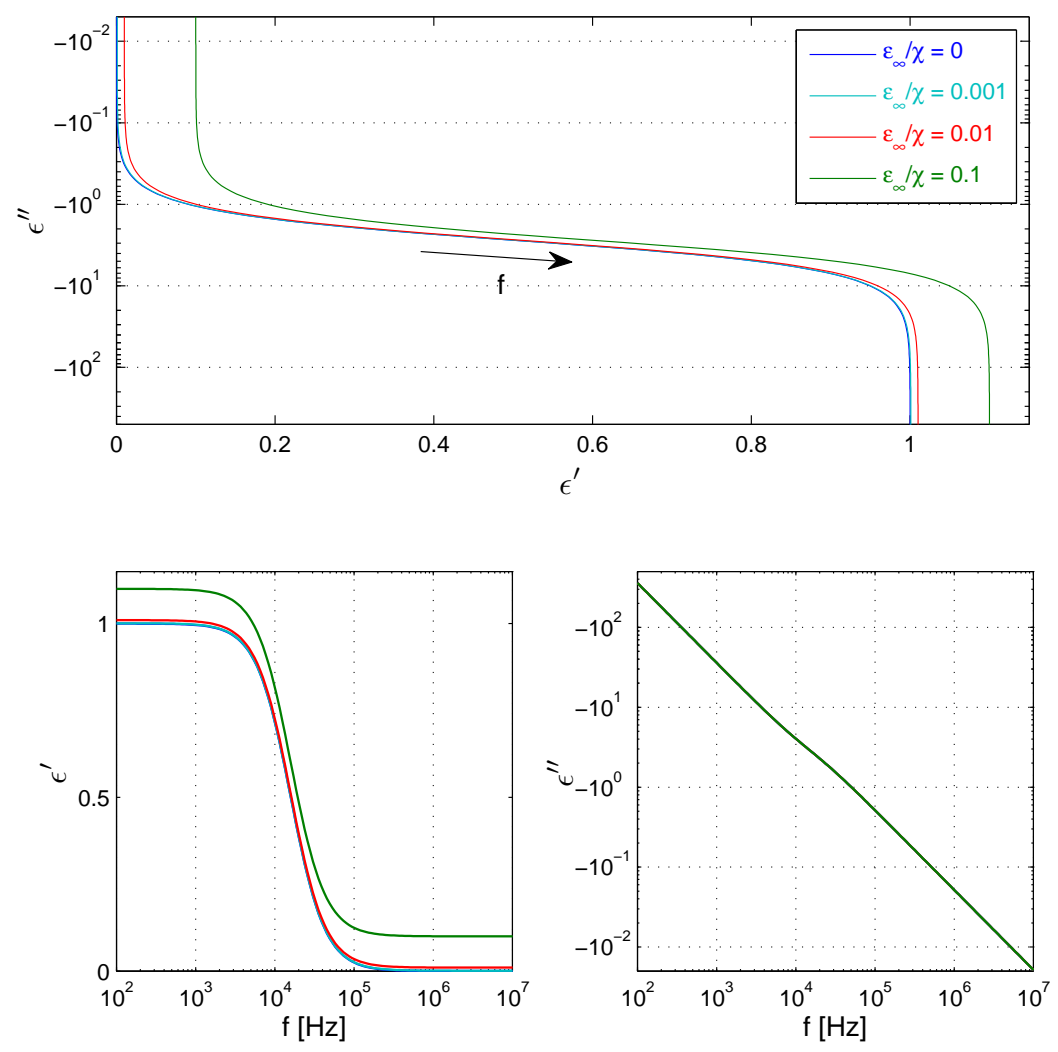

Figure C.2: Complex permittivity representation of a 'lossy' dielectric.

tive, Debye medium can be described by:

$$
\sigma^{*}=j \epsilon_{0} \omega\left(\epsilon_{\infty}+\frac{\chi}{1+j \omega \tau}\right)+\sigma_{D C}
$$

Figure C.3 shows the data of Figure C.2, represented in terms of a complex conductivity.

Provided $\epsilon_{\infty}$ is small then the above equation describes a semicircle in the Argand plane. As $\epsilon_{\infty}$ is increased the imaginary component of the complex conductivity diverges to infinity at high frequencies. The semicircle becomes obscured above $\epsilon / \chi 0.1$. The DC conductivity term 

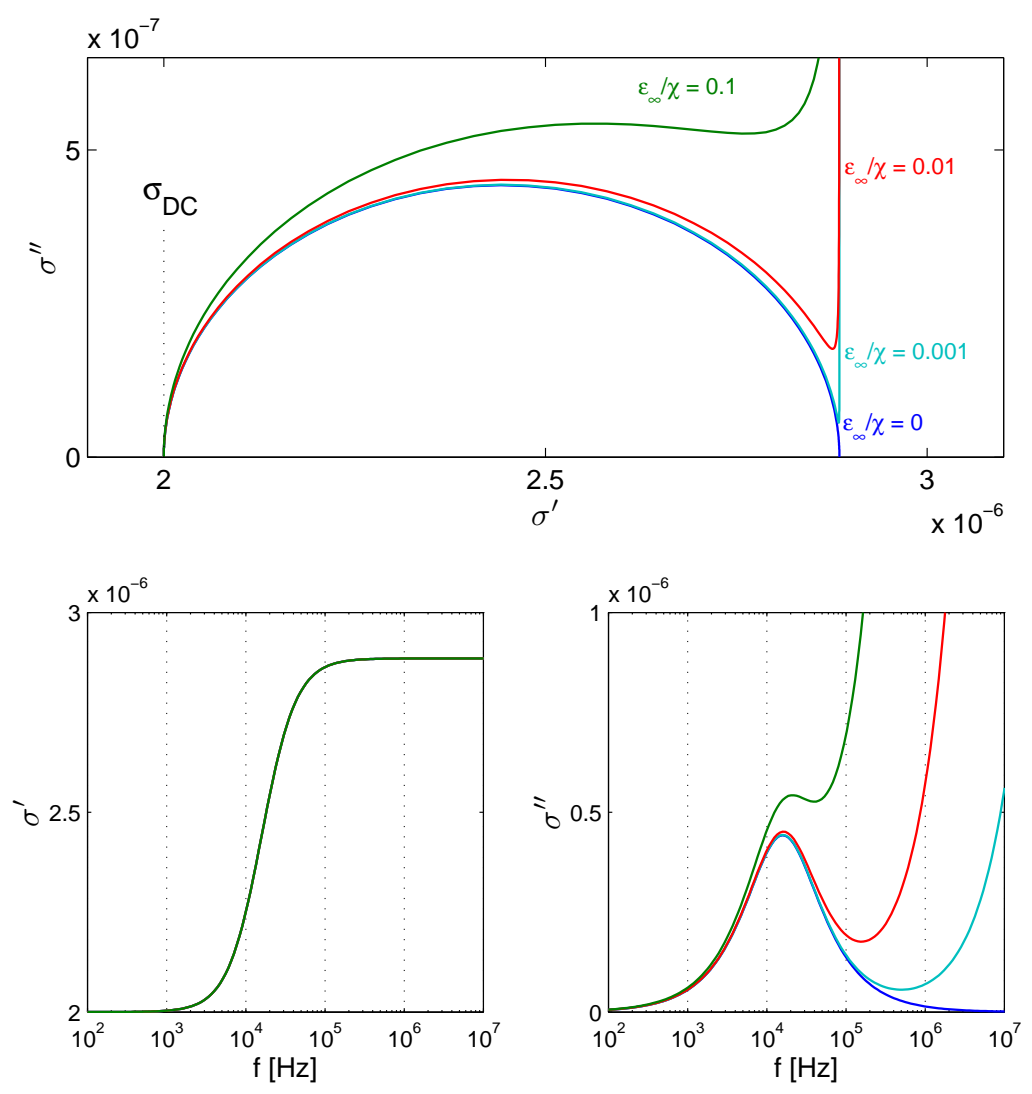

Figure C.3: Complex conductivity representation of a 'lossy' dielectric. The units are $\left[\mathrm{Sm}^{-1}\right]$.

produces a constant offset from the $\mathrm{x}$ axis and, therefore this visualisation is not limited by how small the dielectric loss is compared to the ohmic loss. The relaxation frequency is unchanged by this transformation provided $\epsilon / \chi$ is small. 


\section{Appendix D}

\section{Complex non-linear least squares fitting}

\section{D.1 Theory}

The least squares method determines an approximate solution to an overdetermined set of equations. In its curve fitting usage, a function $y$ with parameters $\mathbf{b}=b_{1}, b_{2}, \ldots, b_{k}$ describes data $\mathbf{Y}$

$$
\begin{aligned}
Y_{1} & =y\left(\omega_{1}, b_{1}, b_{2}, \ldots, b_{k}\right) \\
Y_{2} & =y\left(\omega_{2}, b_{1}, b_{2}, \ldots, b_{k}\right) \\
& \vdots \\
Y_{n} & =y\left(\omega_{n}, b_{1}, b_{2}, \ldots, b_{k}\right) .
\end{aligned}
$$

The goal of the least squares method is to find $\mathbf{b}$ so as to minimise:

$$
\Phi=\sum_{i=1}^{n}\left|Y_{i}-y_{i}\right|^{2}
$$

This formulation is easily generalised to complex data [Tsai and Whitmore (1982)]:

$$
\Phi=\sum_{i=1}^{n}\left[\operatorname{Re}\left(Y_{i}-y_{i}\right)^{2}+\operatorname{Im}\left(Y_{i}-y_{i}\right)^{2}\right]
$$


Tsai and Whitmore (1982) presents a complex non-linear least squares algorithm which takes iterative steps towards a minimum of the above equation. The initial values of $\mathbf{b}$ are guessed and then the following computations are performed to produce new values of $\mathbf{b}$.

$$
\begin{gathered}
P=\left(\frac{\partial y_{i}}{\partial b_{j}}\right), \\
\mathbf{g}=\sum_{i=1}^{n}\left[\operatorname{Re}\left(Y_{i}-y_{i}\right) P_{\operatorname{Re}}+\operatorname{Im}\left(Y_{i}-y_{i}\right) P_{\operatorname{Im}}\right],
\end{gathered}
$$

where $P_{\mathrm{Re}}$ and $P_{\mathrm{Im}}$ are the real and imaginary components of $P$.

$$
\begin{gathered}
A=P_{\operatorname{Re}}^{T} P_{\mathrm{Re}}+P_{\mathrm{Im}}^{T} P_{\mathrm{Im}} \\
\boldsymbol{\delta}=A^{-1} \mathbf{g}
\end{gathered}
$$

The new parameters are then given by:

$$
\mathbf{b}=\mathbf{b}+\boldsymbol{\delta}
$$

This iteration is continued until convergence is achieved.

\section{D.2 6 parameter complex conductivity fit}

A complex non-linear least squares fit was performed on all the wheelie bin experiment complex conductivity data to the following broadband model:

$$
\sigma^{*}=\sigma_{D C}+K(j \omega)^{m}+j \omega \epsilon_{0}\left(\frac{\chi}{1+j \omega \tau}+\epsilon_{\infty}\right) .
$$

Following the formalism outlined in the previous section, the parameter vector $\mathbf{b}$ is then:

$$
\mathbf{b}=\left(\sigma_{D C}, \chi, \tau, \epsilon_{\infty}, K, m\right)
$$

The matrix $P=$

$$
\left|\begin{array}{cccccc}
1 & j \omega_{1} \epsilon_{0} \frac{1}{1+j \omega_{1} \tau} & \omega_{1}^{2} \epsilon_{0} \frac{\chi}{\left(1+j \omega_{1} \tau\right)^{2}} & j \omega_{1} \epsilon_{0} & \left(j \omega_{1}\right)^{m} & K\left(j \omega_{1}\right)^{m} \log \left(j \omega_{1}\right) \\
1 & j \omega_{2} \epsilon_{0} \frac{1}{1+j \omega_{2} \tau} & \omega_{2}^{2} \epsilon_{0} \frac{\chi}{\left(1+j \omega_{2} \tau\right)^{2}} & j \omega_{2} \epsilon_{0} & \left(j \omega_{2}\right)^{m} & K\left(j \omega_{2}\right)^{m} \log \left(j \omega_{2}\right) \\
\vdots & \vdots & \vdots & \vdots & \vdots & \vdots \\
1 & j \omega_{n} \epsilon_{0} \frac{1}{1+j \omega_{n} \tau} & \omega_{n}^{2} \epsilon_{0} \frac{\chi}{\left(1+j \omega_{n} \tau\right)^{2}} & j \omega_{n} \epsilon_{0} & \left(j \omega_{n}\right)^{m} & K\left(j \omega_{n}\right)^{m} \log \left(j \omega_{n}\right)
\end{array}\right|
$$


The matrix $P$ was evaluated in MATLAB which fully supports complex data and so there is no need to evaluate $P_{\operatorname{Re}}$ and $P_{\operatorname{Im}}$ analytically. The complex RMS error of the fit was calculated at each interval.

$$
\lambda_{r m s}=\left(\frac{1}{2} \sum_{i=1}^{n}\left[\operatorname{Re}\left(Y_{i}-y_{i}\right)^{2}+\operatorname{Im}\left(Y_{i}-y_{i}\right)^{2}\right]\right)^{\frac{1}{2}} .
$$

And the inversion of $A$ was stabilised using the Levenburg-Marquadt method:

$$
A=P_{\mathrm{Re}}^{T} P_{\mathrm{Re}}+P_{\mathrm{Im}}^{T} P_{\mathrm{Im}}+\lambda_{r m s}\left(\operatorname{diag}\left(P_{\mathrm{Re}}^{T} P_{\mathrm{Re}}\right)+\operatorname{diag}\left(P_{\mathrm{Im}}^{T} P_{\mathrm{Im}}\right)\right)
$$

The MATLAB code used is included here in its entirety. This code could be placed in a further for loop to automate the fit for different temperature measurements.

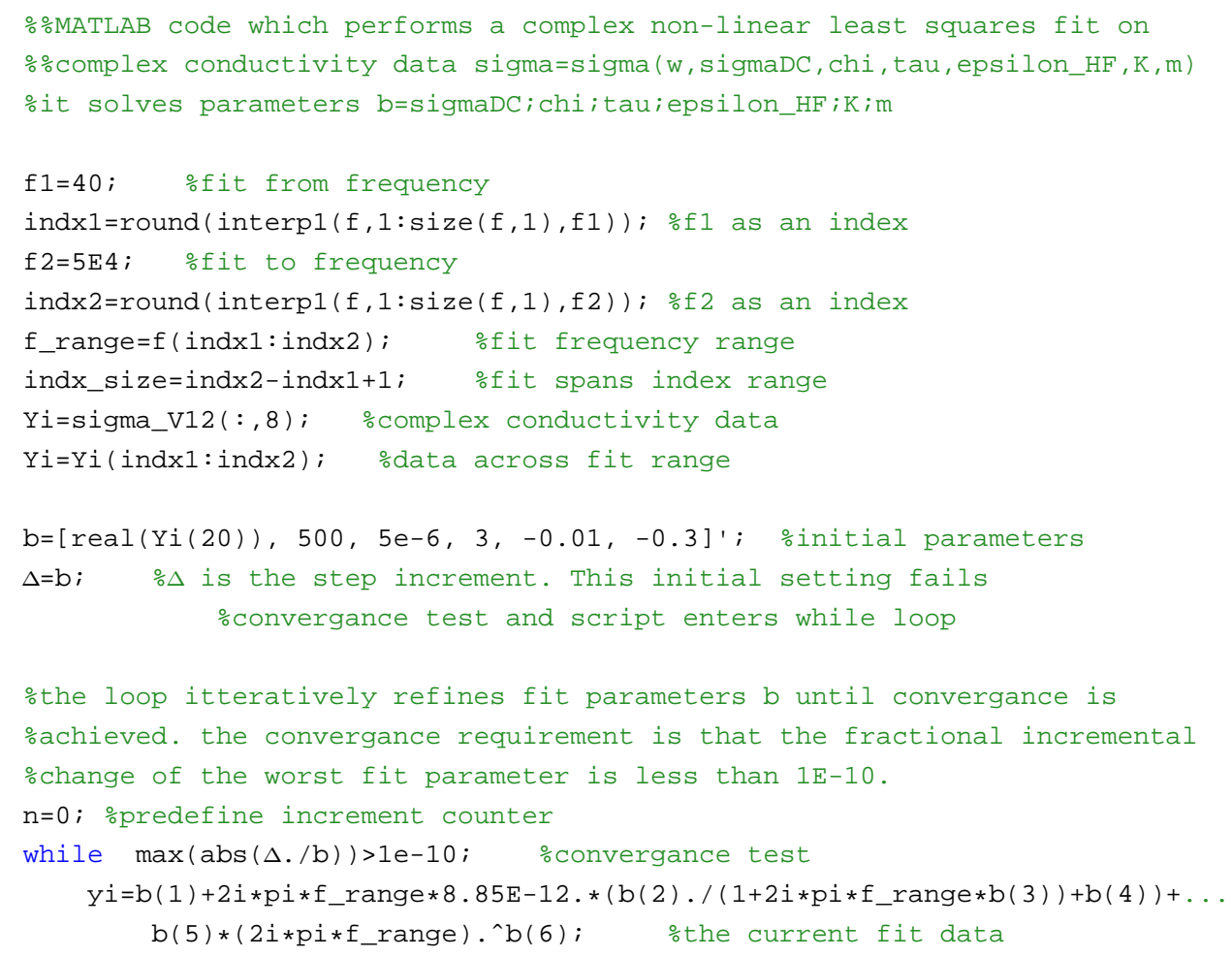




\section{APPENDIX D. COMPLEX NON-LINEAR LEAST SQUARES FITTING130}

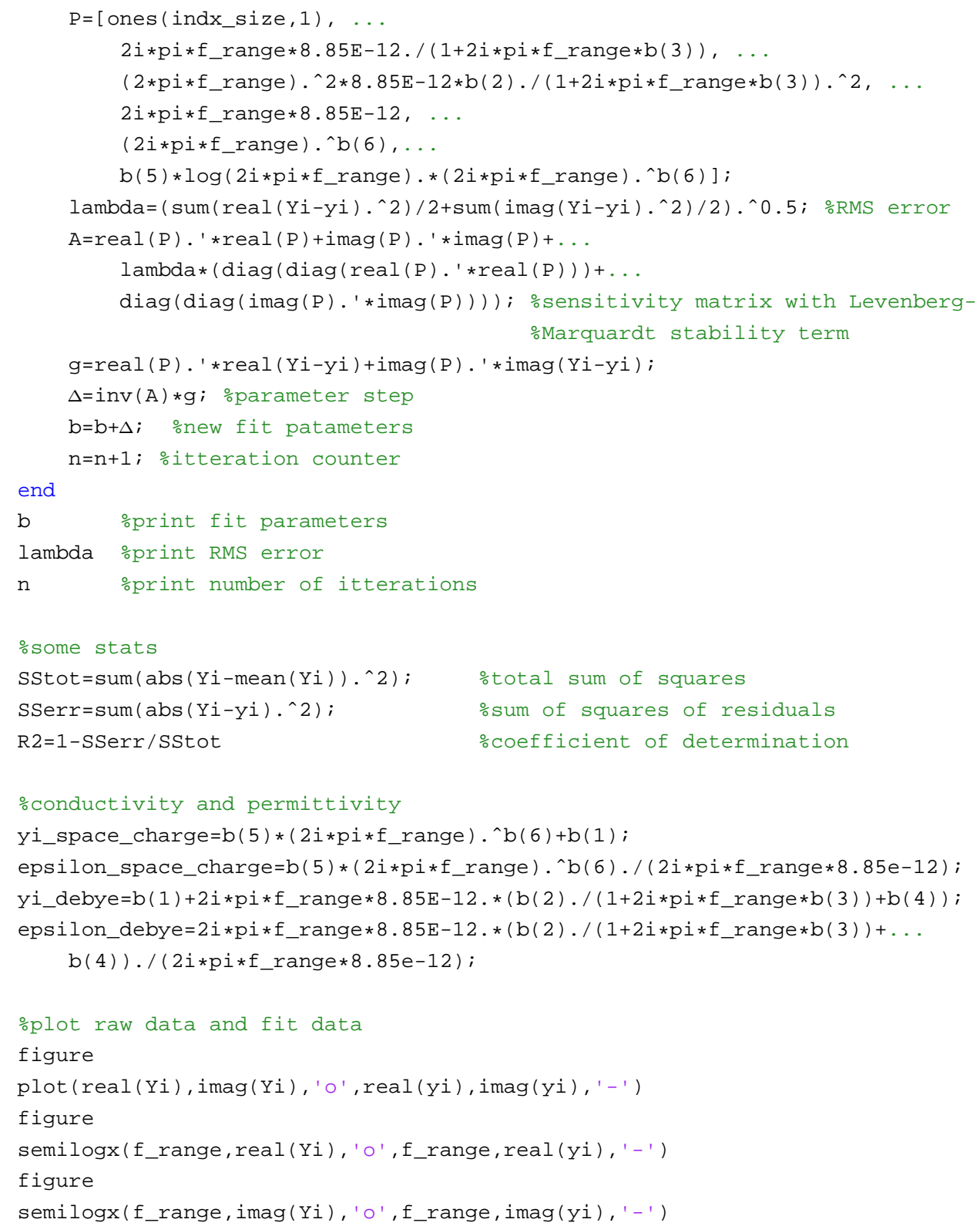




\section{Bibliography}

Addison, J. (1969), Electrical properties of saline ice, Journal of Applied Physics, 40(8), 3105-3114.

Addison, J. (1970), Electrical relaxation in saline ice, Journal of Applied Physics, 41(1), 54-63.

Agilent Technologies (2009), Agilent Impedance Measurement Handbook, 4th ed., Agilent Technologies.

Analog Devices (2009), High performance, $145 \mathrm{mhz}$ fastfet op amps data sheet.

Assur, A. (1958), Composition of sea ice and its tensile strength, in Arctic sea ice: proceedings of the conference, p. 106, National Academy of Sciences, National Research Council.

Backstrom, L., and H. Eicken (2006), Capacitance probe measurements of brine volume and bulk salinity in first-year sea ice, Cold Regions Science and Technology, 46(3), 167-180.

Barsoukov, E., and J. Macdonald (2005), Impedance spectroscopy: theory, experiment, and applications, John Wiley and Sons.

Chelidze, T., Y. Gueguen, and C. Ruffet (1999), Electrical spectroscopy of porous rocks: a review-II. Experimental results and interpretation, Geophysical Journal International, 137(1), 16-34. 
Cox, G., and W. Weeks (1983), Equations for determining the gas and brine volumes in sea ice samples, Journal of Glaciology, 29, 102.

de Loor, G. (1982), Dielectric properties of wet materials, IEEE Transactions on Geoscience and Remote Sensing, GE-21(3), 364-369.

Debye, P. (1945), Polar Molecules, Dover.

Eicken, H. (2003), From the microscopic, to the macroscopic, to the regional scale: growth, microstructure and properties of sea ice, Sea icean introduction to its physics, biology, chemistry and geology. Blackwell Science, London, England, pp. 22-81.

Evans, S. (1965), Dielectric properties of ice and snow-a review, J. Glaciol., vol. 5, p. 773-792 (1965)., 5, 773-792.

Gouws, G., M. Ingham, S. Buchanan, A. Hibbard, A. Mahoney, and A. Gough (2009), Low frequency permittivity measurements of sea ice, in Proceedings of the 8th Annual IEEE Sensors Conference, 25-28 October 2009, Christchurch, New Zealand.

Gouws, G., M. Ingham, S. Buchanan, R. Boyack, A. Mahoney, and A. Gough (2010), Impedance measurement systems for sea ice microstructure determination, in Proceedings of Fourth International Conference on Sensing Technology.

Grimm, R., D. Stillman, S. Dec, and M. Bullock (2008), Low-frequency electrical properties of polycrystalline saline ice and salt hydrates, Journal of Physical Chemistry B, 112(48), 15,382-15,390.

Ingham, M., D. Pringle, and H. Eicken (2008), Cross-borehole resistivity tomography of sea ice, Cold Regions Science and Technology, 52(3), 263277.

Ingham, M., G. Gouws, R. Boyack, and S. Buchanan (2010), In-situ measurement of sea ice permittivity, submitted to Annals of Glaciology. 
Jaccard, C. (1959), Experimental and theoretical study of the electrical properties of ice, Helv. Phys. Acta, 32, 89-128.

Jaccard, C. (1964), Thermodynamics of irreversible processes applied to ice, Zeitschrift fur Physik B Condensed Matter, 3(2), 99-118.

Janz, G., and S. Singer (1975), Copenhangen standard sea water: Conductivity and salinity, Journal of Solution Chemistry, 4(12), 995-1003.

Johari, G., and E. Whalley (1981), The dielectric properties of ice ih in the range 272-133 k, Journal of Chemical Physics, 75, 1333.

Jones, K. A., M. Ingham, and D. J. Pringle (2009), Sea ice microstructure: resolving anisotropic structure through cross-borehole dc resistivity tomography, Journal of Geophysical Research C: Oceans.

Lepparanta, M., and T. Manninen (1988), The brine and gas content of sea ice with attention to low salinities and high temperatures, Finnish Institute of Marine Research, Internal Report, 2, 1-14.

Lide, D. (1993), CRC handbook of chemistry and physics, CRC press.

Macdonald, J. (1953), Theory of ac space-charge polarization effects in photoconductors, semiconductors, and electrolytes, Physical Review, 92(1), 4-17.

Maxwell, J. (1873), A treatise on electricity and magnetism, Clarendon Press.

National Snow and Ice Data Center (2010), Sea ice index.

Notz, D. (2005), Thermodynamic and fluid-dynamical processes in sea ice, Ph.D. thesis, University of Cambridge.

Notz, D., and M. Grae Worster (2008), In situ measurements of the evolution of young sea ice, Journal of Geophysical Research C: Oceans, 113(3). 
Notz, D., J. Wettlaufer, and M. Worster (2005), Instruments and methods a non-destructive method for measuring the salinity and solid fraction of growing sea ice in situ, Journal of Glaciology, 51(172), 159-166.

Petrenko, V., and R. Whitworth (1999), Physics of Ice, Oxford.

Pringle, D. (2004), Thermal conductivity of sea ice and antarctic permafrost, Ph.D. thesis, Victoria University of Wellington.

Pringle, D., G. Dubuis, and H. Eicken (2009), Impedance measurements of the complex dielectric permittivity of sea ice at $50 \mathrm{mhz}$ : Pore microstructure and potential for salinity monitoring, Journal of Glaciology, 55(189), 81-84.

Sillars, R. (1937), The properties of a dielectric containing semi-conducting particles of various shapes, in Proceedings, p. 378.

Stogryn, A., and G. J. Desargant (1985), Dielectric properties of brine in sea ice at microwave frequencies., IEEE Transactions on Antennas and Propagation, AP-33(5), 523-532.

Takei, I., and N. Maeno (1987), Electric characteristics of point defects in HCl-doped ice, Le Journal de Physique Colloques, 48(C1), 1-1.

Tinga, W., W. Voss, and D. Blossey (1973), Generalized approach to multiphase dielectric mixture theory, Journal of Applied Physics, 44(9), 3897-3902.

Tsai, Y., and D. Whitmore (1982), Nonlinear least-squares analyses of complex impedance and admittance data for solid electrolytes, Solid State Ionics, 7(2), 129-139.

Vant, M., R. Ramseier, and V. Makios (1978), The complex-dielectric constant of sea ice at frequencies in the range 0.1-40 ghz, Journal of Applied Physics, 49(3), 1264-1280. 
Wagner, K. (1914), The after effect in dielectrics, Arch. Electrotech, 2, 378394. 